\title{
TANNAKIAN DUALITY FOR ANDERSON-DRINFELD MOTIVES AND ALGEBRAIC INDEPENDENCE OF CARLITZ LOGARITHMS
}

\author{
MATTHEW A. PAPANIKOLAS
}

\begin{abstract}
We develop a theory of Tannakian Galois groups for $t$-motives and relate this to the theory of Frobenius semilinear difference equations. We show that the transcendence degree of the period matrix associated to a given $t$-motive is equal to the dimension of its Galois group. Using this result we prove that Carlitz logarithms of algebraic functions that are linearly independent over the rational function field are algebraically independent.
\end{abstract}

\section{Contents}

1. Introduction

2. Notation and preliminaries

3. $t$-motives and Tannakian categories

4. Galois theory of systems of $\sigma$-semilinear equations 21

5. Galois groups and transcendence 32

6. Application to Carlitz logarithms 33

References

\section{INTRODUCTION}

\subsection{Periods of $t$-motives.}

1.1.1. Notation. Let $\mathbb{F}_{q}$ be the field of $q$ elements, where $q$ is a power of a prime $p$. Let $k:=\mathbb{F}_{q}(\theta)$, where $\theta$ is transcendental over $\mathbb{F}_{q}$, and define an absolute value $|\cdot|_{\infty}$ at the infinite place of $k$ so that $|\theta|_{\infty}=q$. Let $k_{\infty}:=\mathbb{F}_{q}((1 / \theta))$ be the $\infty$-adic completion of $k$, let $\overline{k_{\infty}}$ be an algebraic closure, let $\mathbb{K}$ be the $\infty$-adic completion of $\overline{k_{\infty}}$, and let $\bar{k}$ be the algebraic closure of $k$ in $\mathbb{K}$.

1.1.2. Anderson $t$-motives. Let $t$ be a variable over $\mathbb{F}_{q}$ that is independent from $\theta$, and let $\bar{k}[t ; \boldsymbol{\sigma}]$ be the ring of polynomials in $t$ and $\boldsymbol{\sigma}$ over $\bar{k}$ subject to the relations

$$
c t=t c, \quad \boldsymbol{\sigma} t=t \boldsymbol{\sigma}, \quad \boldsymbol{\sigma} c=c^{1 / q} \boldsymbol{\sigma}, \quad c \in \bar{k} .
$$

An Anderson $t$-motive is a left $\bar{k}[t ; \boldsymbol{\sigma}]$-module $\mathrm{M}$ that is free and finitely generated as both a left $\bar{k}[t]$-module and as a left $\bar{k}[\boldsymbol{\sigma}]$-module and that satisfies $(t-\theta)^{n} \mathrm{M} \subseteq \boldsymbol{\sigma} \mathrm{M}$ for all $n$ sufficiently large (see \$3.4). Anderson $t$-motives were originally defined in [2], where they were called "dual $t$-motives."

Date: June 29, 2007.

2000 Mathematics Subject Classification. Primary: 11J93; Secondary: 11G09, 12H10, 14L17.

Research supported by NSF grant DMS-0340812 and NSA grant MDA904-03-1-0019. 
1.1.3. Rigid analytic triviality. We let $\mathbb{T}:=\mathbb{K}\{t\}$ be the Tate algebra of power series in $\mathbb{K} \llbracket t \rrbracket$ that are convergent on the closed unit disk in $\mathbb{K}$, and let $\mathbb{L} \subseteq \mathbb{K}((t))$ be its fraction field. Let $\mathbb{E}$ be the subring of $\mathbb{T}$ consisting of power series that are everywhere convergent and whose coefficients lie in a finite extension of $k_{\infty}$. Finally, for a Laurent series $f=\sum_{i} a_{i} t^{i} \in \mathbb{K}((t))$ and an integer $n \in \mathbb{Z}$, we set $\sigma^{-n}(f):=f^{(n)}:=\sum_{i} a_{i}^{q^{n}} t^{i}$.

If $\mathrm{M}$ is an Anderson $t$-motive and $\mathrm{m} \in \operatorname{Mat}_{r \times 1}(\mathrm{M})$ has entries comprising a $\bar{k}[t]$-basis of $\mathrm{M}$, then there is a matrix $\Phi \in \operatorname{Mat}_{r}(\bar{k}[t])$ representing multiplication by $\boldsymbol{\sigma}$ on $\mathrm{M}$ so that

$$
\sigma \mathrm{m}=\Phi \mathrm{m}
$$

and $\operatorname{det} \Phi=c(t-\theta)^{s}$ for some $c \in \bar{k}^{\times}$and $s \geq 1$. The Anderson $t$-motive is rigid analytically trivial (see Proposition 3.4.7) if there is a matrix $\Psi \in \mathrm{GL}_{r}(\mathbb{T})$ so that

$$
\Psi^{(-1)}=\Phi \Psi \text {. }
$$

It can be shown that the entries of $\Psi$ are in fact in $\mathbb{E}$ (see Proposition 5.1.3).

1.1.4. Connection with t-modules. The category of rigid analytically trivial Anderson $t$-motives is equivalent to the category of uniformizable abelian $t$-modules defined over $\bar{k}$, as in [1. For a given Anderson $t$-motive $\mathrm{M}$ and associated $t$-module $E$, there is an explicit connection

$$
\text { periods of } E \longleftrightarrow \quad \bar{k} \text {-linear combinations of entries of } \Psi(\theta)^{-1} \text {. }
$$

The details of this relationship will be the subject of a future paper with Anderson, but examples are already seen in $\$ 3.3$ for the Carlitz motive (see also S. K. Sinha [28, §5.2] for examples involving special values of the function field $\Gamma$-function).

1.1.5. Remarks on t-motive terminology. G. Anderson introduced $t$-motives in 1]. Later in 2 dual $t$-motives, which had several technical advantages, were introduced. The algebraic properties of these two types of $t$-motives are essentially the same, and the two categories are anti-equivalent to each other. In this paper we will follow the dual $t$-motive point of view only, and throughout we refer to them as Anderson $t$-motives. In the following paragraph we discuss a third type of $t$-motive, defined properly in 3.4 which are our primary objects of study.

1.1.6. Tannakian category of t-motives. In $\$ 3.4$ we show that the category of rigid analytically trivial Anderson $t$-motives up to isogeny embeds as a full subcategory of a neutral Tannakian category $\mathcal{T}$ over $\mathbb{F}_{q}(t)$. Objects in $\mathcal{T}$ are called simply $t$-motives, and throughout the paper the term "t-motive" will refer exclusively to an object in $\mathcal{T}$. In particular, from this standpoint all $t$-motives are rigid analytically trivial. Also objects in $\mathcal{T}$ do not necessarily come from pure Anderson $t$-motives in the sense of [1], and so $\mathcal{T}$ is a mixed category.

By Tannakian duality, for each object $M$ in $\mathcal{T}$, the Tannakian subcategory $\mathcal{T}_{M}$ generated by $M$ satisfies an equivalence of categories

$$
\mathcal{T}_{M} \approx \operatorname{Rep}\left(\Gamma_{M}, \mathbb{F}_{q}(t)\right),
$$

where $\operatorname{Rep}\left(\Gamma_{M}, \mathbb{F}_{q}(t)\right)$ is the category of finite dimensional representations over $\mathbb{F}_{q}(t)$ of some algebraic subgroup $\Gamma_{M} \subseteq \mathrm{GL}_{r}$ defined over $\mathbb{F}_{q}(t)$ (see $\$ 3.5$ ). The group $\Gamma_{M}$ is called the Galois group of $M$.

It should be noted that R. Pink 23 , has defined a category $\mathcal{H}$ of mixed Hodge structures for function fields that is a neutral Tannakian category over $\mathbb{F}_{q}(t)$. He showed that the category of rigid analytically trivial Anderson $t$-motives that are also "mixed" embeds as a full subcategory of $\mathcal{H}$. It would be interesting to investigate the relationships among Pink's Hodge structures, the $t$-motives defined in this paper, and their associated 
Galois groups. In the end our category of $t$-motives is best suited for our transcendence applications, so we do not pursue further here the connections with Pink's work. See also D. Goss [14 for additional comparisons between $t$-motives and motives over $\mathbb{Q}$.

The following is the main theorem of this paper (restated later as Theorem [5.2.2).

Theorem 1.1.7. Let $M$ be a t-motive, and let $\Gamma_{M}$ be its Galois group. Suppose that $\Phi \in \mathrm{GL}_{r}(\bar{k}(t)) \cap \operatorname{Mat}_{r}(\bar{k}[t])$ represents multiplication by $\boldsymbol{\sigma}$ on $M$ and that $\operatorname{det} \Phi=$ $c(t-\theta)^{s}, c \in \bar{k}^{\times}$. Let $\Psi$ be a rigid analytic trivialization of $\Phi$ in $\mathrm{GL}_{r}(\mathbb{T}) \cap \operatorname{Mat}_{r}(\mathbb{E})$. Finally, let $L$ be the subfield of $\overline{k_{\infty}}$ generated over $\bar{k}$ by the entries of $\Psi(\theta)$. Then

$$
\text { tr. } \operatorname{deg}_{\bar{k}} L=\operatorname{dim} \Gamma_{M} \text {. }
$$

1.1.8. Grothendieck's conjecture. In light of \$1.1.4 the statement of Theorem 1.1.7 can be thought of as a function field version of Grothendieck's conjecture on periods of algebraic varieties. For an abelian variety $A$ over $\overline{\mathbb{Q}}$ of dimension $d$, let $P$ be the period matrix of $A$ that represents an isomorphism between $H^{1}(A(\mathbb{C}), \mathbb{Q}) \otimes_{\mathbb{Q}} \mathbb{C}$ and $H_{\mathrm{DR}}^{1}(A / \mathbb{C})$, with basis defined over $\overline{\mathbb{Q}}$. Grothendieck's conjecture is that

$$
\text { tr. } \operatorname{deg}_{\overline{\mathbb{Q}}} \overline{\mathbb{Q}}(P)=\operatorname{dim} \operatorname{MT}(A),
$$

where $\operatorname{MT}(A)$ is the Mumford-Tate group of $A$ and is an algebraic subgroup of $\mathrm{GL}_{2 d} \times \mathbb{G}_{\mathrm{m}}$ over $\mathbb{Q}$. P. Deligne [11, Cor. I.6.4] has proved that the dimension of $\mathrm{MT}(A)$ is an upper bound for the transcendence degree. Conjecturally the Mumford-Tate group is isomorphic to the motivic Galois group of the motive $h_{1}(A) \oplus \mathbb{Q}(1)$ over $\mathbb{Q}$. More generally Grothendieck's period conjecture states that if $X$ is a smooth variety over $\overline{\mathbb{Q}}$, then

$$
\text { tr. } \operatorname{deg}_{\overline{\mathbb{Q}}} \overline{\mathbb{Q}}(P(X))=\operatorname{dim} \Gamma_{X}^{\operatorname{mot}},
$$

where $P(X)$ is the period matrix of $X$ and $\Gamma_{X}^{\text {mot }}$ is the motivic Galois group of $X$ over $\mathbb{Q}$. It should be pointed out that by work of $\mathrm{C}$. Bertolin [5] many standard transcendence conjectures over $\overline{\mathbb{Q}}$, such as Schanuel's conjecture, follow from expanded versions of Grothendieck's period conjecture.

1.2. Algebraic independence of Carlitz logarithms. One application of Theorem 1.1.7 is a characterization of algebraic relations over $\bar{k}$ of Carlitz logarithms of algebraic numbers.

1.2.1. Carlitz exponential. The Carlitz exponential is the power series

$$
\exp _{C}(z):=z+\sum_{i=1}^{\infty} \frac{z^{q^{i}}}{\left(\theta^{q^{i}}-\theta\right)\left(\theta^{q^{i}}-\theta^{q}\right) \cdots\left(\theta^{q^{i}}-\theta^{q^{i-1}}\right)} .
$$

As is well known (see [15, Ch. 3], [31, §2.5]), the function defined by $\exp _{C}$ converges everywhere on $\mathbb{K}$, is $\mathbb{F}_{q}$-linear, and has kernel $\mathbb{F}_{q}[\theta] \tilde{\pi}$, where

$$
\tilde{\pi}:=\theta \sqrt[q-1]{-\theta} \prod_{i=1}^{\infty}\left(1-\theta^{1-q^{i}}\right)^{-1} \in k_{\infty}(\sqrt[q-1]{-\theta})^{\times} .
$$

The Carlitz exponential also satisfies the functional equation

$$
\exp _{C}(\theta z)=\theta \exp _{C}(z)+\exp _{C}(z)^{q}, \quad z \in \mathbb{K} .
$$

Moreover, this functional equation induces an exact sequence of $\mathbb{F}_{q}[t]$-modules,

$$
0 \rightarrow \mathbb{F}_{q}[\theta] \widetilde{\pi} \rightarrow \mathbb{K} \rightarrow \mathfrak{C}(\mathbb{K}) \rightarrow 0
$$

where $\mathfrak{C}(\mathbb{K})$ is the $\mathbb{F}_{q}[t]$-module of $\mathbb{K}$-valued points on the Carlitz module $\mathfrak{C}$ (see 93.4 .4 ) and where $t$ acts by multiplication by $\theta$ on the first two terms. The number $\widetilde{\pi}$ is called the Carlitz period. 
1.2.2. Carlitz logarithm. The Carlitz logarithm is the inverse of $\exp _{C}(z)$,

$$
\log _{C}(z):=z+\sum_{i=1}^{\infty} \frac{z^{q^{i}}}{\left(\theta-\theta^{q}\right)\left(\theta-\theta^{q^{2}}\right) \cdots\left(\theta-\theta^{q^{i}}\right)},
$$

which as a function on $\mathbb{K}$ converges for all $z \in \mathbb{K}$ with $|z|_{\infty}<|\theta|_{\infty}^{q /(q-1)}$. The Carlitz logarithm is $\mathbb{F}_{q}$-linear and satisfies the functional equation

$$
\theta \log _{C}(z)=\log _{C}(\theta z)+\log _{C}\left(z^{q}\right),
$$

for all $z \in \mathbb{K}$ where all three terms converge.

1.2.3. Linear forms in Carlitz logarithms. We recall a theorem of J. Yu. Suppose $\lambda_{1}, \ldots, \lambda_{r} \in \mathbb{K}$ satisfy $\exp _{C}\left(\lambda_{i}\right) \in \bar{k}$ for each $i=1, \ldots, r$. As in the previous section there are many potential $k$-linear relations among $\lambda_{1}, \ldots, \lambda_{r}$. However, Yu proved that these are the only possible linear relations over $\bar{k}$ in the following function field analogue of Baker's theorem on linear forms in logarithms.

Theorem 1.2.4 (Yu [33, Thm. 4.3]). Suppose $\lambda_{1}, \ldots, \lambda_{r} \in \mathbb{K}$ satisfy $\exp _{C}\left(\lambda_{i}\right) \in \bar{k}$ for $i=1, \ldots, r$. If $\lambda_{1}, \ldots, \lambda_{r}$ are linearly independent over $k$, then the numbers $1, \lambda_{1}, \ldots, \lambda_{r}$ are linearly independent over $\bar{k}$.

Yu's result is an application of his far reaching Theorem of the Sub-t-module 33 Thm. 0.1], which characterizes all $\bar{k}$-linear relations among logarithms of points in $\bar{k}$ on general $t$-modules. Transcendence results about the Carlitz periods and Carlitz logarithms go back to Carlitz and Wade in the 1940's. For detailed accounts of the history of transcendence results for Drinfeld modules, including Yu's theorem, see W. D. Brownawell [9] and D. S. Thakur [31, Ch. 10].

1.2.5. Algebraic independence of Carlitz logarithms. In characteristic 0, Baker's theorem on linear forms in natural logarithms of algebraic numbers is best known. In the situation of Carlitz logarithms we use Theorem 1.1.7 to prove the following theorem (restated later as Theorem 6.4.2).

Theorem 1.2.6. Let $\lambda_{1}, \ldots, \lambda_{r} \in \mathbb{K}$ satisfy $\exp _{C}\left(\lambda_{i}\right) \in \bar{k}$ for each $i=1, \ldots, r$. If $\lambda_{1}, \ldots, \lambda_{r}$ are linearly independent over $k$, then they are algebraically independent over $\bar{k}$.

It should be noted that, using Mahler's method, L. Denis [12] has proved the special case of this theorem where $\lambda_{1}, \ldots, \lambda_{r}$ are restricted to values of $\log _{C}$ on elements of $\mathbb{F}_{q}\left(\theta^{1 / e}\right), e \geq 1$, of degree in $\theta$ less than $q /(q-1)$.

\subsection{Methods of proof.}

1.3.1. $\sigma$-semilinear difference equations. The category of $t$-motives is a certain full subcategory in the category of left $\bar{k}(t)\left[\boldsymbol{\sigma}, \boldsymbol{\sigma}^{-1}\right]$-modules which are finite dimensional as $\bar{k}(t)$-vector spaces. To every $t$-motive $M$ one can associate a matrix $\Phi \in \mathrm{GL}_{r}(\bar{k}(t))$ representing multiplication by $\sigma$ and a rigid analytic trivialization $\Psi \in \mathrm{GL}_{r}(\mathbb{L})$ so that $\Psi^{(-1)}=\Phi \Psi$. Here recall that $\mathbb{L}$ is the fraction field of the Tate algebra $\mathbb{T}$. Thus the columns of $\Psi$ satisfy a system of $\sigma$-semilinear difference equations in the sense of [25], where $\sigma=\left(f \mapsto f^{(-1)}\right): \mathbb{L} \stackrel{\sim}{\rightarrow} \mathbb{L}$, and we develop the theory of such equations in this context in 4 . In spirit this theory is close to the Galois theory of differential equations and difference equations in characteristic 0 [4, 6], 10], 20, 24, 25], 26].

In $\$ 4$ we develop the Picard-Vessiot theory for certain kinds of difference equations for $\sigma$ and construct their difference Galois groups (see Theorem 4.2.11). However, careful attention must be paid to the fact that the fixed field of $\sigma$ in $\bar{k}(t)$ is $\mathbb{F}_{q}(t)$. The Galois 
theory of difference equations developed by M. van der Put and M. F. Singer 25 is quite useful here, but it does not completely apply because they fundamentally use that the field of fixed elements under the difference automorphism is algebraically closed. On the one hand, because the fixed field of $\sigma$ in $\mathbb{L}$ is also $\mathbb{F}_{q}(t)$, the Galois groups we construct are themselves defined over $\mathbb{F}_{q}(t)$. However, that $\mathbb{F}_{q}(t)$ is not algebraically closed nor even perfect presents several difficulties because in general the $\mathbb{F}_{q}(t)$-valued points of the Galois group need not be dense and the group itself need not be a priori smooth.

1.3.2. $t$-motives and difference Galois groups. Given a $t$-motive $M$ of dimension $r$ over $\bar{k}(t)$, the difference Galois group $\Gamma$ is a subgroup of $\mathrm{GL}_{r}$ over $\mathbb{F}_{q}(t)$. Let $\Sigma$ be the $\bar{k}(t)$ subalgebra of $\mathbb{L}$ generated by the entries of $\Psi$ and $\operatorname{det}(\Psi)^{-1}$, and let $\Lambda$ be its fraction field. The field $\mathbb{L}$ is naturally a left $\bar{k}(t)\left[\boldsymbol{\sigma}, \boldsymbol{\sigma}^{-1}\right]$-module via the automorphism $\sigma$, and $\Sigma$ and $\Lambda$ are both $\sigma$-invariant. Then

$$
\Gamma\left(\mathbb{F}_{q}(t)\right) \cong \operatorname{Aut}_{\sigma}(\Sigma / \bar{k}(t)),
$$

where the right-hand side is the group of automorphisms of $\Sigma$ over $\bar{k}(t)$ that commute with $\sigma$. Moreover, this identification is compatible with base extensions of $\mathbb{F}_{q}(t)$ (see 4.4.14.4.3).

We work out an explicit description of $\Gamma\left(\overline{\mathbb{F}_{q}(t)}\right)$ in 4.4 , and, using crucially that $\mathbb{L}$ is a separable extension of $\bar{k}(t)$ and that $\bar{k}(t)$ is algebraically closed in $\Lambda$, we show that $\Gamma$ has the following properties:

- $\Gamma$ is smooth over $\mathbb{F}_{q}(t)$ (Theorem 4.3.1(b));

- $\operatorname{dim} \Gamma=$ tr. $\operatorname{deg}_{\bar{k}(t)} \Lambda$, (Theorem 4.3.1(c));

- The elements of $\Lambda$ fixed by $\Gamma\left(\overline{\mathbb{F}_{q}(t)}\right)$ are precisely $\bar{k}(t)$ (Theorem 4.4 .6 ).

These properties are essential for proving in Theorem 4.5.10 that

$$
\Gamma \cong \Gamma_{M},
$$

where $\Gamma_{M}$ is the Galois group associated to $M$ by Tannakian duality.

1.3.3. The proof of Theorem 1.1.7. The primary vehicle for proving this theorem is a $\bar{k}$-linear independence criterion from [2, Thm. 3.1.1]. It is stated here in Theorem 5.1.1. We apply this criterion to the rigid analytic trivializations of tensor powers of $M$ so as to compare the dimensions of the $\bar{k}$-span of monomials of the entries of $\Psi(\theta)$ of a given degree and the $\bar{k}(t)$-span of monomials in the entries of $\Psi$. Ultimately we show that

$$
\text { tr. } \operatorname{deg}_{\bar{k}} L=\text { tr. } \operatorname{deg}_{\bar{k}(t)} \Lambda,
$$

the latter of which is the same as the dimension of $\Gamma_{M}$.

1.3.4. Carlitz logarithms. For $\alpha_{1}, \ldots, \alpha_{r} \in \bar{k}^{\times}$with $\left|\alpha_{i}\right|_{\infty}<|\theta|_{\infty}^{q /(q-1)}$ for $i=1, \ldots, r$, we define a $t$-motive $X$ so that the field generated over $\bar{k}$ by the entries of its rigid analytic trivialization $\Psi$ evaluated at $t=\theta$ is precisely

$$
L=\bar{k}(\Psi(\theta))=\bar{k}\left(\tilde{\pi}, \log _{C}\left(\alpha_{1}\right), \ldots, \log _{C}\left(\alpha_{r}\right)\right) .
$$

Moreover, we show that arbitrary logarithms are $k$-linear combinations of logarithms of this form in a precise way. We determine a set of defining equations of the Galois group $\Gamma_{X}$ of $X$ in Theorem 6.3.2 each of which is a linear polynomial over $\bar{k}(t)$. These linear relations each produce a $k$-linear relation on the logarithms and $\widetilde{\pi}$. We then use Theorem 1.1.7 to show that all algebraic relations must arise from these relations. 
1.4. Acknowledgements. The author thanks D. Brownawell, L. Denis, C.-Y. Chang, D. Goss, L.-C. Hsia, M. van der Put, D. Thakur, and J. Yu for many helpful discussions on the contents of this paper. He further thanks the National Center for Theoretical Sciences in Hsinchu, Taiwan, where many of the results in this paper were proved. The author especially thanks G. Anderson and N. Ramachandran for their indispensable advice throughout this project. Finally the author thanks the referee for several useful suggestions.

\section{Notation AND PRELiminaries}

\subsection{Table of symbols.}

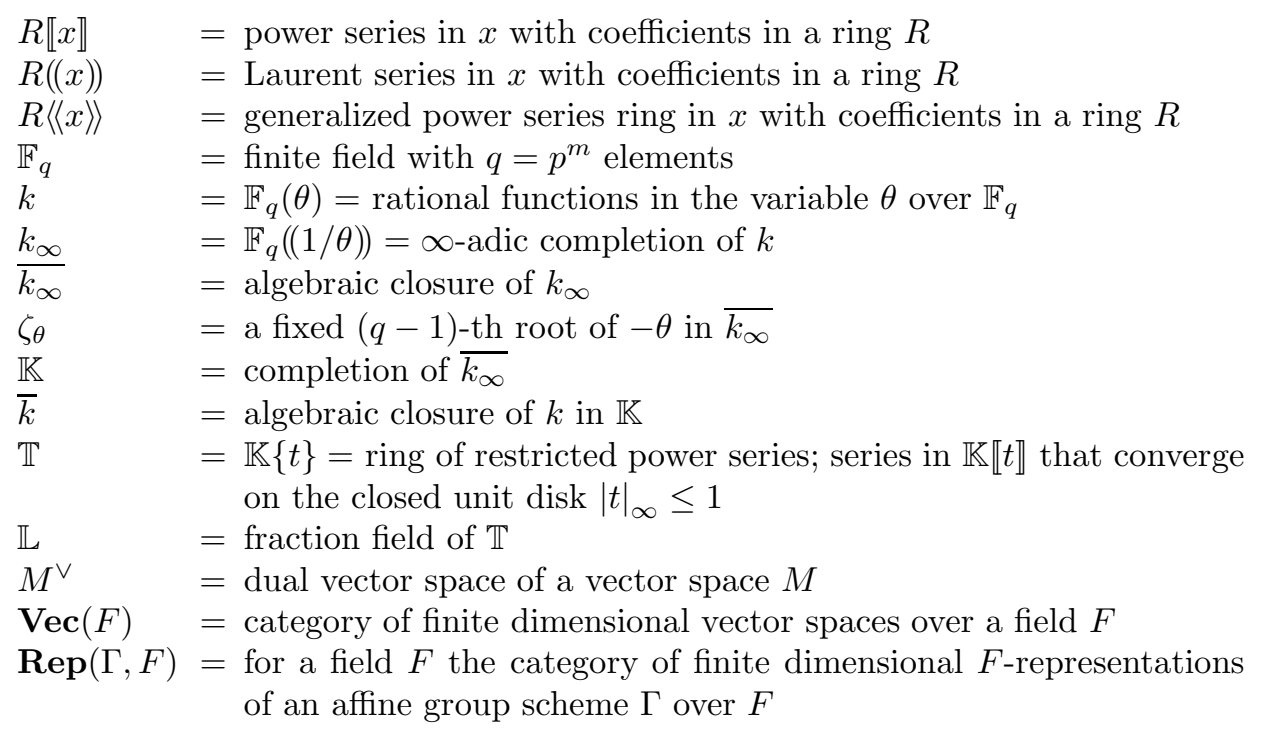

\subsection{Preliminaries.}

2.2.1. Norms. We let $|\cdot|_{\infty}$ denote a fixed $\infty$-adic norm on $\mathbb{K}$. For a matrix $E \in$ $\operatorname{Mat}_{r \times s}(\mathbb{K})$, we set $|E|_{\infty}=\sup \left|E_{i j}\right|_{\infty}$. For matrices $E$ and $F$, we observe that $|E+F|_{\infty} \leq \max \left(|E|_{\infty},|F|_{\infty}\right)$ and $|E F|_{\infty} \leq|E|_{\infty} \cdot|F|_{\infty}$.

2.2.2. Generalized power series. Let $F$ be a field of characteristic $p$. For a formal series $f:=\sum_{i \in \mathbb{Q}} a_{i} t^{i}$ with $a_{i} \in F$, we let $\operatorname{Supp}(f):=\left\{i \in \mathbb{Q} \mid a_{i} \neq 0\right\}$. We let $F\langle\langle t\rangle\rangle$ be the set of such series for which $\operatorname{Supp}(f)$ is a well-ordered subset of $\mathbb{Q}$. This condition implies that $F\langle\langle t\rangle\rangle$ is a field under the natural addition and multiplication of these series so that $t^{i} t^{j}=t^{i+j}$ (see P. Ribenboim [27, §2]). If $F$ is algebraically closed, then $F\langle\langle t\rangle\rangle$ is algebraically closed [27, §5]. If $F$ is a perfect field, then $F\langle\langle t\rangle\rangle$ is also perfect.

It should be noted that, when $F$ is algebraically closed, $F\langle\langle t\rangle\rangle$ is not the algebraic closure of the Laurent series field $F((t))$. For an explicit description of the field $\overline{F((t))} \subseteq$ $F\langle\langle t\rangle\rangle$, the reader is directed to K. Kedlaya [17].

By considering the inclusions

$$
\mathbb{F}_{q}(t) \subseteq \bar{k}(t) \subseteq \mathbb{K}((t)) \subseteq \mathbb{K}\langle\langle t\rangle\rangle,
$$

we fix once and for all the inclusions of algebraically closed fields

$$
\overline{\mathbb{F}_{q}(t)} \subseteq \overline{k(t)} \subseteq \overline{\mathbb{K}((t))} \subseteq \mathbb{K}\langle\langle t\rangle\rangle
$$


2.2.3. Entire functions. A power series $f=\sum_{i=0}^{\infty} a_{i} t^{i} \in \mathbb{K} \llbracket t \rrbracket$ that satisfies

$$
\lim _{i \rightarrow \infty} \sqrt[i]{\left|a_{i}\right|_{\infty}}=0
$$

and

$$
\left[k_{\infty}\left(a_{0}, a_{1}, a_{2}, \ldots\right): k_{\infty}\right]<\infty,
$$

is an entire power series. As a function of $t$, such a power series $f$ converges on all of $\mathbb{K}$, and, when restricted to $\overline{k_{\infty}}, f$ takes values in $\overline{k_{\infty}}$. The ring of entire power series is denoted $\mathbb{E}$.

2.2.4. Restricted Laurent series. A power series $\sum_{i=0}^{\infty} a_{i} t^{i} \in \mathbb{K} \llbracket t \rrbracket$ that satisfies

$$
\lim _{i \rightarrow \infty}\left|a_{i}\right|_{\infty}=0 \text {, }
$$

is called a restricted power series. As functions of $t$, these power series converge on the closed unit disk in $\mathbb{K}$. The restricted power series form a subring $\mathbb{T}=\mathbb{K}\{t\}$ of $\mathbb{K} \llbracket t \rrbracket$, and $\mathbb{E}$ is a subring of $\mathbb{T}$. The fraction field of $\mathbb{T}$, denoted $\mathbb{L}$, is the field of restricted Laurent series.

Now at each point $a \in \mathbb{K}$ with $|a|_{\infty} \leq 1$, a function $f \in \mathbb{L}$ has a well-defined order of vanishing $\operatorname{ord}_{a}(f)$, and for all but finitely many $|a|_{\infty} \leq 1$, we have $\operatorname{ord}_{a}(f)=0$. Also each $f \in \mathbb{L}$ has a unique factorization

$$
f=\lambda\left[\prod_{|a|_{\infty} \leq 1}(t-a)^{\operatorname{ord}_{a}(f)}\right]\left[1+\sum_{i=1}^{\infty} b_{i} t^{i}\right]
$$

where $0 \neq \lambda \in \mathbb{K}$, sup $\left|b_{i}\right|_{\infty}<1$, and $\left|b_{i}\right|_{\infty} \rightarrow 0$ (see [13. Cor. 2.2.4]). The series $1+\sum b_{i} t^{i}$ is a unit in $\mathbb{T}$, and it follows that $\mathbb{T}$ is a principal ideal domain with maximal ideals generated by each $t-a,|a|_{\infty} \leq 1$ (see [13, Thm. 2.2.9]).

For $f=\sum_{i=0}^{\infty} a_{i} t^{i} \in \mathbb{T}$, we define its norm $\|f\|$ to be

$$
\|f\|:=\sup _{i}\left|a_{i}\right|_{\infty}=\max _{i}\left|a_{i}\right|_{\infty}
$$

If $f \in \mathbb{T}$ is written as in (2.2.4.1), then $\|f\|=|\lambda|_{\infty}$. The norm $\|\cdot\|$ is a complete ultrametric norm on $\mathbb{T}$ and satisfies

$$
\begin{aligned}
\|c f\| & =|c|_{\infty}\|f\|, & & \forall c \in \mathbb{K}, f \in \mathbb{T}, \\
\|f g\| & =\|f\| \cdot\|g\|, & & \forall f, g \in \mathbb{T} .
\end{aligned}
$$

2.2.5. Twisting. We define an automorphism $\sigma: \mathbb{K}\langle\langle t\rangle\rangle \rightarrow \mathbb{K}\langle\langle t\rangle\rangle$ by setting

$$
\sigma\left(\sum_{i \in \mathbb{Q}} a_{i} t^{i}\right):=\sum_{i \in \mathbb{Q}} a_{i}^{1 / q} t^{i}
$$

If $f \in \mathbb{K}\langle\langle t\rangle\rangle$ and $n \in \mathbb{Z}$, the $n$-fold twist of $f$ is defined to be

$$
f^{(n)}:=\sigma^{-n}(f)
$$

The automorphism $\sigma$ of $\mathbb{K}\langle\langle t\rangle\rangle$ induces automorphisms of several subrings, notably $\bar{k}[t]$, $\bar{k}(t), \mathbb{E}, \mathbb{T}, \mathbb{L}, \mathbb{K} \llbracket t \rrbracket, \mathbb{K}((t))$. Moreover, $\sigma$ also leaves $\overline{\mathbb{F}_{q}(t)}, \overline{k(t)}, \overline{\mathbb{L}}$, and $\overline{\mathbb{K}((t))}$ invariant.

If $F$ is a subring of $\mathbb{K}\langle\langle t\rangle\rangle$ that is invariant under $\sigma$, we set

$$
F^{\sigma}:=\{f \in F \mid \sigma(f)=f\}
$$


to be the elements of $F$ fixed by $\sigma$. It is clear that $F^{\sigma^{n}}$ is a subring of $F$ and that $F^{\sigma^{m}} \subseteq F^{\sigma^{n}}$ if $m \mid n$. For example,

$$
\begin{array}{ll}
\mathbb{K}\langle\langle t\rangle\rangle^{\sigma}=\mathbb{F}_{q}\langle\langle t\rangle\rangle, & \overline{k(t)}=\overline{\mathbb{F}}_{q}^{\sigma}\left({ }^{\sigma}=\overline{\mathbb{F}_{q}(t)} \cap \mathbb{F}_{q}\langle\langle t\rangle\rangle,\right. \\
\mathbb{K}((t))^{\sigma}=\mathbb{F}_{q}((t)), & \bar{k}(t)^{\sigma}=\mathbb{F}_{q}(t) .
\end{array}
$$

The only item that requires any explanation here is the description of $\overline{k(t)}^{\sigma}$. For $\alpha \in \overline{k(t)}^{\sigma}$, let $x^{m}+b_{m-1} x^{m-1}+\cdots+b_{0} \in \bar{k}(t)[x]$ be the minimal polynomial of $\alpha$ over $\bar{k}(t)$. Since $\sigma(\alpha)=\alpha$, we have that $\alpha$ is also a root of $x^{m}+\sigma\left(b_{m-1}\right) x^{m-1}+\cdots+\sigma\left(b_{0}\right)$. Taking the difference of these two relations, we see that $\sigma\left(b_{i}\right)=b_{i}$ for each $i$, and so the minimal polynomial of $\alpha$ has coefficients in $\bar{k}(t)^{\sigma}=\mathbb{F}_{q}(t)$.

If $F$ is a matrix with entries in $\mathbb{K}\langle\langle t\rangle\rangle$, then $\sigma^{-n}(F):=F^{(n)}$ is defined by the rule $\left(F^{(n)}\right)_{i j}:=\left(F_{i j}^{(n)}\right)$. If $F \in \operatorname{Mat}_{r \times s}(\mathbb{L})$, we set $\|F\|:=\max _{i, j}\left\|F_{i j}\right\|$, in which case $\left\|F^{(n)}\right\|=\|F\|^{q^{n}}$.

Lemma 2.2.6. For any $\alpha \in \mathbb{K}$, there is a positive integer $s$ so that with respect to $|\cdot|_{\infty}$ on $\mathbb{K}$,

$$
\lim _{n \rightarrow \infty} \alpha^{(n s)}= \begin{cases}0 & \text { if }|\alpha|_{\infty}<1 \\ c \in \overline{\mathbb{F}}_{q}^{\times} & \text {if }|\alpha|_{\infty}=1 \\ \infty & \text { if }|\alpha|_{\infty}>1\end{cases}
$$

Proof. If $|\alpha|_{\infty} \neq 1$, then the result is clear. Otherwise, there is a unique $c \in \overline{\mathbb{F}}_{q}^{\times}$so that $|\alpha-c|_{\infty}<1$. (See [28, Lem. 2.4.4].) Then $c \in \mathbb{F}_{q^{s}}$ for some $s \geq 1$, and the result follows.

Lemma 2.2.7. For any $f \in \mathbb{T}$ with $\|f\| \leq 1$, there is a positive integer $s$ so that with respect to $\|\cdot\|$ on $\mathbb{T}$,

$$
\lim _{n \rightarrow \infty} f^{(n s)} \in \overline{\mathbb{F}}_{q}[t]
$$

Also $\|f\|=1$ if and only if $\lim _{n \rightarrow \infty} f^{(n s)} \neq 0$.

Proof. We use the factorization of $f$ in (2.2.4.1). For each $a$, with $|a|_{\infty} \leq 1$, if $\operatorname{ord}_{a}(f) \neq$ 0 , then as in Lemma 2.2.6 choose $s_{a} \geq 1$ and $c_{a} \in \overline{\mathbb{F}}_{q}$ so that $\lim _{n \rightarrow \infty} a^{\left(n s_{a}\right)}=c_{a}$. Likewise, since $\|f\| \leq 1$, we have $|\lambda|_{\infty} \leq 1$, and so we can choose $s_{\lambda} \geq 1$ and $c_{\lambda} \in \overline{\mathbb{F}}_{q}$ with $\lambda^{\left(n s_{\lambda}\right)} \rightarrow c_{\lambda}$. Then we let $s$ be the least common multiple of all the $s_{a}$ 's and $s_{\lambda}$. From (2.2.4.1), with respect to $\|\cdot\|$,

$$
\lim _{n \rightarrow \infty}\left[1+\sum_{i=1}^{\infty} b_{i} t^{i}\right]^{(n s)}=1,
$$

since $\sup \left|b_{i}\right|_{\infty}<1$. Therefore,

$$
\lim _{n \rightarrow \infty} f^{(n s)}=\lim _{n \rightarrow \infty} \lambda^{(n s)} \prod_{|a|_{\infty} \leq 1}\left(t-a^{(n s)}\right)^{\operatorname{ord}_{a}(f)}=c_{\lambda} \prod_{|a|_{\infty} \leq 1}\left(t-c_{a}\right)^{\operatorname{ord}_{a}(f)},
$$

which is in $\overline{\mathbb{F}}_{q}[t]$. Furthermore, $\|f\|=1$ if and only if $|\lambda|_{\infty}=1$, which holds if and only if $c_{\lambda} \neq 0$. Thus $\|f\|=1$ if and only if $\lim _{n \rightarrow \infty} f^{(n s)} \neq 0$. 


\section{3. $t$-MOTIVES AND TANNAKIAN CATEGORIES}

Here we will define a category $\mathcal{T}$ of $t$-motives that is a neutral Tannakian category over $\mathbb{F}_{q}(t)$. For all definitions of tensor categories and Tannakian categories, we follow Deligne and J. S. Milne [11, §II]. Other useful references include [8, 10, 26, App. B].

As mentioned in 11.1.6. Tannakian categories for $t$-motives have been considered previously by Pink 23], though through a different construction. Parts of the theory of $t$-motives defined below have been considered by Y. Taguchi [29] and A. Tamagawa [30] in their study of the Tate conjecture for $t$-modules. Also our theory has similarities with the theory of $\sigma$-bundles defined by U. Hartl and Pink [16].

\subsection{The rings $\bar{k}[t ; \boldsymbol{\sigma}]$ and $\bar{k}(t)\left[\boldsymbol{\sigma}, \boldsymbol{\sigma}^{-1}\right]$.}

3.1.1. Definition. The $\operatorname{ring} \bar{k}(t)\left[\boldsymbol{\sigma}, \boldsymbol{\sigma}^{-1}\right]$ is the noncommutative ring of Laurent polynomials in the variable $\boldsymbol{\sigma}$ with coefficients in $\bar{k}(t)$, subject to the relation

$$
\boldsymbol{\sigma} f=\sigma(f) \boldsymbol{\sigma}=f^{(-1)} \boldsymbol{\sigma}
$$

for all $f \in \bar{k}(t)$. Thus every element of $\bar{k}(t)\left[\boldsymbol{\sigma}, \boldsymbol{\sigma}^{-1}\right]$ has the form $\sum_{i=-m}^{m} f_{i} \boldsymbol{\sigma}^{i}$, where $f_{i} \in \bar{k}(t)$.

3.1.2. Ring-theoretic properties. The polynomials in $\sigma$ with coefficients in $\bar{k}[t]$ comprise the subring $\bar{k}[t ; \boldsymbol{\sigma}]$ of $\bar{k}(t)\left[\boldsymbol{\sigma}, \boldsymbol{\sigma}^{-1}\right]$. The $\operatorname{ring} \bar{k}[\boldsymbol{\sigma}]$ is the subring of polynomials with coefficients in $\bar{k}$. Both $\bar{k}[t ; \boldsymbol{\sigma}]$ and $\bar{k}(t)\left[\boldsymbol{\sigma}, \boldsymbol{\sigma}^{-1}\right]$ are domains. The center of $\bar{k}[t ; \boldsymbol{\sigma}]$ is $\mathbb{F}_{q}[t]$, and the center of $\bar{k}(t)\left[\boldsymbol{\sigma}, \boldsymbol{\sigma}^{-1}\right]$ is $\mathbb{F}_{q}(t)$. The fundamental properties of the ring $\bar{k}[t ; \boldsymbol{\sigma}]$ are covered in $[2, \S 4]$.

3.2. Pre-t-motives. Here we define the category $\mathcal{P}$ of pre-t-motives and explore its basic properties. In particular we show in Theorem 3.2.13 that $\mathcal{P}$ is a rigid abelian $\mathbb{F}_{q}(t)$-linear tensor category.

3.2.1. The category $\mathcal{P}$. We let $\mathcal{P}$ be the category of left $\bar{k}(t)\left[\boldsymbol{\sigma}, \boldsymbol{\sigma}^{-1}\right]$-modules that are finite dimensional over $\bar{k}(t)$. Morphisms in $\mathcal{P}$ are left $\bar{k}(t)\left[\boldsymbol{\sigma}, \boldsymbol{\sigma}^{-1}\right]$-module homomorphisms. We call $\mathcal{P}$ the category of pre-t-motives, though it is worth noting that $\mathcal{P}$ is a category of difference modules with respect to the automorphism $\sigma: \bar{k}(t) \rightarrow \bar{k}(t)$ in the sense of [25].

3.2.2. Preliminary properties of $\mathcal{P}$. The category of pre- $t$-motives is an abelian category. For two objects $P$ and $Q$ in $\mathcal{P}$, it follows that $\operatorname{Hom}_{\mathcal{P}}(P, Q)$ is an $\mathbb{F}_{q}(t)$-vector space. A straightforward adaptation of the proof of [1, Thm. 2] shows that the map

$$
\operatorname{Hom}_{\mathcal{P}}(P, Q) \otimes_{\mathbb{F}_{q}(t)} \bar{k}(t) \rightarrow \operatorname{Hom}_{\bar{k}(t)}(P, Q)
$$

is injective. Thus $\operatorname{Hom}_{\mathcal{P}}(P, Q)$ is a finite dimensional $\mathbb{F}_{q}(t)$-vector space.

3.2.3. Representations of pre-t-motives. Given a $\bar{k}(t)$-vector space $P$ and $p_{1}, \ldots, p_{r} \in P$, we call the vector

$$
\mathbf{p}=\left[\begin{array}{c}
p_{1} \\
\vdots \\
p_{r}
\end{array}\right] \in \operatorname{Mat}_{r \times 1}(P)
$$

a basis for $P$ if $p_{1}, \ldots, p_{r}$ form a $\bar{k}(t)$-basis for $P$. If $P$ is a pre- $t$-motive, then there is a unique matrix $\Phi=\Phi_{\mathbf{p}} \in \mathrm{GL}_{r}(\bar{k}(t))$ such that

$$
\sigma \mathbf{p}=\Phi \mathbf{p}
$$


We say that $\Phi$ represents multiplication by $\sigma$ on $P$. Moreover, the matrix $\Phi \in \mathrm{GL}_{r}(\bar{k}(t))$ uniquely determines the left $\bar{k}(t)\left[\boldsymbol{\sigma}, \boldsymbol{\sigma}^{-1}\right]$-module structure on $P$ with respect to $\mathbf{p}$.

Now suppose that $\phi: P \rightarrow Q$ is a morphism of pre- $t$-motives and that $\mathbf{p} \in \operatorname{Mat}_{r \times 1}(P)$ and $\mathbf{q} \in \operatorname{Mat}_{s \times 1}(Q)$ are bases for $P$ and $Q$ respectively. If $B \in \operatorname{Mat}_{r \times s}(\bar{k}(t))$ represents $\phi$ as a map of $\bar{k}(t)$-vector spaces such that

$$
\phi(\mathbf{f} \cdot \mathbf{p})=\mathbf{f} \cdot B \cdot \mathbf{q}, \quad \mathbf{f} \in \operatorname{Mat}_{1 \times r}(\bar{k}(t)),
$$

then

$$
B^{(-1)} \Phi_{\mathbf{q}}=\Phi_{\mathbf{p}} B
$$

In particular, if $\mathbf{q}$ is simply another basis of $P$, and $B \in \mathrm{GL}_{r}(\bar{k}(t))$ is the change of basis matrix, then $\Phi_{\mathbf{p}}=B^{(-1)} \Phi_{\mathbf{q}} B^{-1}$.

3.2.4. Tensor products of pre-t-motives. Let $P$ and $Q$ be pre-t-motives. Then the $\bar{k}(t)-$ vector space $P \otimes_{\bar{k}(t)} Q$ is made into a $\bar{k}(t)\left[\boldsymbol{\sigma}, \boldsymbol{\sigma}^{-1}\right]$-module by defining

$$
\boldsymbol{\sigma}(m \otimes n):=(\boldsymbol{\sigma} m) \otimes(\boldsymbol{\sigma} n) .
$$

It is clear that then multiplication by $\boldsymbol{\sigma}$ is bijective on $P \otimes_{\bar{k}(t)} Q$ and that $P \otimes_{\bar{k}(t)} Q$ is a pre-t-motive. Likewise we define arbitrary finite tensor products of pre- $t$-motives with diagonal $\sigma$-action. For a fixed pre-t-motive $P$ and $n \geq 1$, we set $P^{\otimes n}:=\bigotimes_{i=1}^{n} P$ to be the $n$-th tensor power of $P$.

3.2.5. Representations of tensor products. Let $\mathbf{p}=\left[p_{1}, \ldots, p_{r}\right]^{\operatorname{tr}}$ and $\mathbf{q}=\left[q_{1}, \ldots, q_{s}\right]^{\operatorname{tr}}$ be $\bar{k}(t)$-bases for pre-t-motives $P$ and $Q$ respectively. Then, with respect to the basis

$$
\mathbf{p} \otimes \mathbf{q}:=\left[p_{1} \otimes q_{1}, p_{1} \otimes q_{2}, \ldots, p_{r} \otimes q_{s}\right]^{\mathrm{tr}},
$$

on $P \otimes Q$, the Kronecker product, $\Phi_{\mathbf{p} \otimes \mathbf{q}}=\Phi_{\mathbf{p}} \otimes \Phi_{\mathbf{q}}$, represents multiplication by $\boldsymbol{\sigma}$ on $P \otimes Q$. Similarly these conventions extend to arbitrary finite tensor products of pre- $t$-motives.

3.2.6. The Carlitz motive. We define the Carlitz motive to be the pre- $t$-motive $C$ whose underlying $\bar{k}(t)$-vector space is $\bar{k}(t)$ itself and on which $\boldsymbol{\sigma}$ acts by

$$
\boldsymbol{\sigma} f:=(t-\theta) f^{(-1)}, \quad f \in C .
$$

For $n \geq 1$, the underlying $\bar{k}(t)$-vector space of $C^{\otimes n}$ is also $\bar{k}(t)$, and multiplication by $\boldsymbol{\sigma}$ on $C^{\otimes n}$ is given by

$$
\boldsymbol{\sigma} f=(t-\theta)^{n} f^{(-1)}, \quad f \in C^{\otimes n} .
$$

See also [3].

3.2.7. Internal Hom. Let $P$ and $Q$ be pre- $t$-motives, and set

$$
R:=\operatorname{Hom}_{\bar{k}(t)}(P, Q) .
$$

Then $R$ is a $\bar{k}(t)$-vector space. We define a $\bar{k}(t)\left[\boldsymbol{\sigma}, \boldsymbol{\sigma}^{-1}\right]$-module structure on $R$ by setting

$$
\boldsymbol{\sigma} \cdot \rho:=\boldsymbol{\sigma} \circ \rho \circ \boldsymbol{\sigma}^{-1}, \quad \rho \in R .
$$

It is straightforward to check that $\boldsymbol{\sigma} \cdot \rho: P \rightarrow Q$ is $\bar{k}(t)$-linear, and so $\boldsymbol{\sigma}: R \rightarrow R$, and that this action of $\boldsymbol{\sigma}$ extends naturally to a left $\bar{k}(t)\left[\boldsymbol{\sigma}, \boldsymbol{\sigma}^{-1}\right]$-module structure on $R$. We write $\operatorname{Hom}(P, Q)$ for the $\bar{k}(t)\left[\boldsymbol{\sigma}, \boldsymbol{\sigma}^{-1}\right]$-module $R$ just defined. It is also a pre- $t$-motive. 
3.2.8. Identity object. Let $\mathbf{1}:=\bar{k}(t)$ together with a $\boldsymbol{\sigma}$-action defined by

$$
\boldsymbol{\sigma} f=\sigma(f)=f^{(-1)}, \quad f \in \mathbf{1} .
$$

Then 1 is a pre-t-motive. Moreover, for any pre- $t$-motive $P$, the natural isomorphisms, $P \otimes \mathbf{1} \cong \mathbf{1} \otimes P \cong P$, are isomorphisms of pre-t-motives. Thus $\mathbf{1}$ is an identity object with respect to tensor products in $\mathcal{P}$.

Lemma 3.2.9. $\operatorname{End}_{\mathcal{P}}(1) \cong \mathbb{F}_{q}(t)$.

Proof. Suppose $\phi: \mathbf{1} \rightarrow \mathbf{1}$ is a morphism in $\mathcal{P}$. As a map of $\bar{k}(t)$-vector spaces, there is some $a \in \bar{k}(t)$ so that $\phi(f)=a f$ for all $f \in \bar{k}(t)$. Since $\phi$ is also $\bar{k}(t)\left[\boldsymbol{\sigma}, \boldsymbol{\sigma}^{-1}\right]$-linear, we must have $\boldsymbol{\sigma} a=a \boldsymbol{\sigma}$, and so $a$ is in the center of $\bar{k}(t)\left[\boldsymbol{\sigma}, \boldsymbol{\sigma}^{-1}\right]$. Thus $a \in \mathbb{F}_{q}(t)$.

3.2.10. Duals. Let $P$ be a pre-t-motive. Then set

$$
P^{\vee}:=\operatorname{Hom}(P, \mathbf{1}) \text {. }
$$

The pre-t-motive $P^{\vee}$ is called the dual of $P$. As a $\bar{k}(t)$-vector space, $P^{\vee}$ is the dual vector space of $P$. If $\mathbf{p}$ forms a basis for $P$, let $\mathbf{p}^{\vee}$ be the dual basis. We find easily that

$$
\Phi_{\mathbf{p} v}=\left(\Phi_{\mathbf{p}}^{-1}\right)^{\operatorname{tr}}
$$

If $\phi: P \rightarrow Q$ is a morphism of pre-t-motives, then the dual morphism of $\bar{k}(t)$-vector spaces, $\phi^{\vee}: Q^{\vee} \rightarrow P^{\vee}$, is also $\bar{k}(t)\left[\boldsymbol{\sigma}, \boldsymbol{\sigma}^{-1}\right]$-linear. These constructions are functorial in $P$ and $Q$, and thus $P \mapsto P^{\vee}: \mathcal{P} \rightarrow \mathcal{P}$ defines a contravariant $\mathbb{F}_{q}(t)$-linear functor.

3.2.11. Dual of the Carlitz motive. Using the definition of the Carlitz motive in \$3.2.6. we see that $C^{\vee}$ is isomorphic to $\bar{k}(t)$ as a $\bar{k}(t)$-vector space and that

$$
\boldsymbol{\sigma} f=\frac{1}{t-\theta} \cdot f^{(-1)}, \quad f \in C^{\vee}(=\bar{k}(t)) .
$$

Furthermore, we see that $C^{\vee} \otimes C \cong \mathbf{1}$ and that $C$ is an invertible object in $\mathcal{P}$. Thus the functor

$$
P \mapsto P \otimes C: \mathcal{P} \rightarrow \mathcal{P}
$$

is an equivalence of categories. We define for $n \in \mathbb{Z}$,

$$
C(n):= \begin{cases}C^{\otimes n} & \text { if } n>0, \\ \mathbf{1} & \text { if } n=0, \\ \left(C^{\vee}\right)^{\otimes-n} & \text { if } n<0 .\end{cases}
$$

3.2.12. Rigid abelian tensor category. In the language of [11, §II.1], it is easily shown that the category of pre- $t$-motives is an abelian $\mathbb{F}_{q}(t)$-linear tensor category. We omit the details, but we observe that

- each $\operatorname{Hom}_{\mathcal{P}}(P, Q)$ is a finite dimensional vector space over $\mathbb{F}_{q}(t)$;

- $\otimes$ is compatibly associative and commutative;

- $\otimes$ is $\mathbb{F}_{q}(t)$-bilinear;

- $\mathbf{1}$ is an identity object with respect to tensor products.

Furthermore, it is straightforward to check that

- the pre-t-motive $\operatorname{Hom}(P, Q)$ defines an internal Hom in $\mathcal{P}$ that is compatible with tensor products;

- for each pre-t-motive $P$, there is a natural isomorphism $P \cong P^{\vee \vee}$.

Therefore, $\mathcal{P}$ is also rigid. We record this information in the following theorem.

Theorem 3.2.13. The category $\mathcal{P}$ of pre-t-motives is a rigid abelian $\mathbb{F}_{q}(t)$-linear tensor category. 


\subsection{Rigid analytic triviality.}

3.3.1. The category $\mathcal{R}$. Let $P$ be a pre- $t$-motive. We set

$$
P^{\dagger}:=\mathbb{L} \otimes_{\bar{k}(t)} P,
$$

and give $P^{\dagger}$ a left $\bar{k}(t)\left[\boldsymbol{\sigma}, \boldsymbol{\sigma}^{-1}\right]$-module structure by setting

$$
\boldsymbol{\sigma}(f \otimes m):=f^{(-1)} \otimes \boldsymbol{\sigma} m .
$$

Let

$$
P^{\mathrm{B}}:=\left(P^{\dagger}\right)^{\boldsymbol{\sigma}}=\left\{\mu \in P^{\dagger} \mid \boldsymbol{\sigma} \mu=\mu\right\} .
$$

Then $P^{\mathrm{B}}$ is an $\mathbb{F}_{q}(t)$-vector space, and $P \mapsto P^{\mathrm{B}}$ is a covariant functor from $\mathcal{P}$ to the category of $\mathbb{F}_{q}(t)$-vector spaces. (The "B" in $P^{\mathrm{B}}$ stands for "Betti.") It is straightforward to check that $P \mapsto P^{\mathrm{B}}$ is left exact.

We say that $P$ is rigid analytically trivial if the natural map

$$
\mathbb{L} \otimes_{\mathbb{F}_{q}(t)} P^{\mathrm{B}} \rightarrow P^{\dagger}
$$

is an isomorphism. If $P \cong Q$ as pre-t-motives and $P$ is rigid analytically trivial, then so is $Q$. We let $\mathcal{R}$ denote the strictly full subcategory of $\mathcal{P}$ whose objects are the rigid analytically trivial pre- $t$-motives. Clearly the zero object is rigid analytically trivial, and so $\mathcal{R}$ is non-empty. We shall see momentarily that $\mathbf{1}$ and $C$ are also rigid analytically trivial.

Lemma 3.3.2. We have $\mathbb{L}^{\sigma}=\mathbb{F}_{q}(t)$.

Proof. By definition, for $f \in \mathbb{L}^{\sigma}$ we have $f^{(-1)}=f$, and so by (2.2.4.1) the polar divisor $D$ of $f$ on the closed unit disk in $\mathbb{K}$ must also satisfy $D^{(-1)}=D$. Therefore $D$ is the divisor of zeros of a polynomial $c$ in $\mathbb{F}_{q}[t]$. Then $c f \in \mathbb{T}$, and $(c f)^{(-1)}=c f$, from which we have $c f \in \mathbb{T} \cap \mathbb{F}_{q} \llbracket t \rrbracket=\mathbb{F}_{q}[t]$.

Proposition 3.3.3. The pre-t-motive 1 is rigid analytically trivial.

Proof. It is clear that $\mathbf{1}^{\dagger}=\mathbb{L}$ with $\boldsymbol{\sigma} f:=f^{(-1)}$ for $f \in \mathbb{L}$. Therefore, by Lemma 3.3.2, $\mathbf{1}^{\mathrm{B}}=\mathbb{L}^{\sigma}=\mathbb{F}_{q}(t)$. Thus $\mathbb{L} \otimes_{\mathbb{F}_{q}(t)} \mathbf{1}^{\mathrm{B}} \cong \mathbf{1}^{\dagger}$.

3.3.4. The power series $\Omega$. Consider the power series

$$
\Omega=\Omega(t):=\zeta_{\theta}^{-q} \prod_{i=1}^{\infty}\left(1-t / \theta^{(i)}\right) \in k_{\infty}\left(\zeta_{\theta}\right) \llbracket t \rrbracket \subseteq \mathbb{K} \llbracket t \rrbracket .
$$

It is not difficult to show that $\Omega(t)$ has an infinite radius of convergence, and so $\Omega \in$ $\mathbb{E} \subseteq \mathbb{T}$. Since $\Omega$ has infinitely many zeros in $\mathbb{K}$, it follows that $\Omega \notin \overline{\mathbb{K}(t)}$. Since $\Omega$ has no zeros inside the unit disk, it follows that $\Omega \in \mathbb{T}^{\times}$. It also satisfies the functional equation

The number

$$
\Omega^{(-1)}=(t-\theta) \Omega .
$$

$$
\widetilde{\pi}=-\frac{1}{\Omega(\theta)}=\theta \zeta_{\theta} \prod_{i=1}^{\infty}\left(1-\theta^{1-q^{i}}\right)^{-1} \in k_{\infty}\left(\zeta_{\theta}\right)
$$

is the Carlitz period, which figures prominently in our transcendence considerations later on (see also [3, Cor. 5.2.8], [15, §3.2], [31, §2.5]).

Lemma 3.3.5. Suppose $f \in \mathbb{L}$ satisfies $(t-\theta)^{n} f^{(-1)}=f$ for some $n \in \mathbb{Z}$. Then $f=c / \Omega^{n}$ for some $c \in \mathbb{F}_{q}(t)$.

Proof. Let $c=f \Omega^{n}$. Then $c$ satisfies $c^{(-1)}=c$, and so by Lemma 3.3.2, $c \in \mathbb{F}_{q}(t)$. 
Proposition 3.3.6. The Carlitz motive $C$ is rigid analytically trivial.

Proof. We see that $C^{\dagger}=\mathbb{L}$ with $\boldsymbol{\sigma} f=(t-\theta) f^{(-1)}$ for $f \in \mathbb{L}$. Therefore, by Lemma 3.3.5

$$
C^{\mathrm{B}}=\left\{f \in \mathbb{L} \mid(t-\theta) f^{(-1)}=f\right\}=\frac{1}{\Omega} \cdot \mathbb{F}_{q}(t) .
$$

Therefore $\mathbb{L} \otimes_{\mathbb{F}_{q}(t)} C^{\mathrm{B}} \cong C^{\dagger}$.

Lemma 3.3.7. Let $P$ be a pre-t-motive, and let $\mu_{1}, \ldots, \mu_{m} \in P^{\mathrm{B}}$. If $\mu_{1}, \ldots, \mu_{m}$ are linearly independent over $\mathbb{F}_{q}(t)$, then they are linearly independent over $\mathbb{L}$ in $P^{\dagger}$.

Proof. Suppose that $m \geq 2$ is minimal such that $\mu_{1}, \ldots, \mu_{m}$ are linearly independent over $\mathbb{F}_{q}(t)$ but that $\sum_{i=1}^{m} f_{i} \mu_{i}=0$, with $f_{i} \in \mathbb{L}, f_{1}=1$. Now,

$$
\boldsymbol{\sigma} \sum_{i=1}^{m} f_{i} \mu_{i}=\sum_{i=1}^{m} f_{i}^{(-1)} \mu_{i}=0 .
$$

Therefore, $\sum_{i=2}^{m}\left(f_{i}-f_{i}^{(-1)}\right) \mu_{i}=0$. By the minimality of $m$ and Lemma 3.3.2, each $f_{i}$ is in $\mathbb{F}_{q}(t)$. However, this violates the $\mathbb{F}_{q}(t)$-linear independence of $\mu_{1}, \ldots, \mu_{m}$.

Proposition 3.3.8. If $P$ is a pre-t-motive, then $\operatorname{dim}_{\mathbb{F}_{q}(t)} P^{\mathrm{B}} \leq \operatorname{dim}_{\bar{k}(t)} P$. Equality holds if and only if $P$ is rigid analytically trivial.

Proof. From Lemma 3.3.7 the map $\mathbb{L} \otimes_{\mathbb{F}_{q}(t)} P^{\mathrm{B}} \rightarrow P^{\dagger}$ is injective. The inequality in the statement of the proposition follows from the equality $\operatorname{dim}_{\bar{k}(t)} P=\operatorname{dim}_{\mathbb{L}} P^{\dagger}$. By the definition of rigid analytic triviality, equality holds if and only if the map above is also surjective.

Proposition 3.3.9. Suppose that $P$ is a pre-t-motive and that $\Phi$ represents multiplication by $\boldsymbol{\sigma}$ on $P$ with respect to the basis $\mathbf{p}$ of $P$.

(a) $P$ is rigid analytically trivial if and only if there is a matrix $\Psi \in \mathrm{GL}_{r}(\mathbb{L})$ satisfying

$$
\sigma(\Psi)=\Psi^{(-1)}=\Phi \Psi
$$

Such a matrix $\Psi$ is called a rigid analytic trivialization of $\Phi$ (cf. [1, Thm. 5], [2, Lem. 4.4.13]).

(b) If $\Psi$ is a rigid analytic trivialization of $\Phi$, then the entries of $\Psi^{-1} \mathbf{p}$ form an $\mathbb{F}_{q}(t)$-basis for $P^{\mathrm{B}}$.

(c) If $P$ is rigid analytically trivial, $\Phi \in \operatorname{Mat}_{r}(\bar{k}[t])$, and $\operatorname{det}(\Phi)=d(t-\theta)^{s}$ for some $s \geq 0$ and $d \in \bar{k}^{\times}$, then there is a rigid analytic trivialization $\Psi$ of $\Phi$ with $\Psi \in \mathrm{GL}_{r}(\mathbb{T})$.

Proof. The proofs of parts (a) and (b) are essentially the same as the proof of 2 , Lem. 4.4.13] with minor modifications. We provide a sketch for completeness. ((a) $\Leftarrow$; (b)): Certainly if we have such a $\Psi$, then the entries of $\Psi^{-1} \mathbf{p}$ are both an $\mathbb{L}$-basis of $P^{\dagger}$ and also an $\mathbb{F}_{q}(t)$-linearly independent set in $P^{\mathrm{B}}$. By Proposition 3.3.8 the entries of $\Psi^{-1} \mathbf{p}$ must be an $\mathbb{F}_{q}(t)$-basis of $P^{\mathrm{B}}$, and thus $P$ is rigid analytically trivial. $(($ a) $\Rightarrow)$ : On the other hand, if $P$ is rigid analytically trivial, then there is a matrix $\Theta \in G_{r}(\mathbb{L})$ so that the entries of $\Theta \mathbf{p}$ are both an $\mathbb{L}$-basis of $P^{\dagger}$ and an $\mathbb{F}_{q}(t)$-basis of $P^{\mathrm{B}}$. Setting $\Psi:=\Theta^{-1}$ gives the desired matrix.

For part (c), we first let $\mathrm{P}$ be the $\bar{k}[t]$-span of the entries of $\mathbf{p}$, and set

$$
\mathrm{P}^{\dagger}:=\mathbb{T} \otimes_{\bar{k}[t]} \mathrm{P}, \quad \mathrm{P}^{\mathrm{B}}:=\left\{\mu \in \mathrm{P}^{\dagger} \mid \boldsymbol{\sigma} \mu=\mu\right\} .
$$


For $\mu \in P^{\mathrm{B}}$, write $\mu=\sum f_{i} p_{i}=\mathbf{f} \cdot \mathbf{p}$ with $\mathbf{f} \in \operatorname{Mat}_{1 \times r}(\mathbb{L})$. We claim that for some $c \in \mathbb{F}_{q}[t]$, we have $c \mu \in \mathrm{P}^{\mathrm{B}}$. Let $\operatorname{den}(\mathbf{f}) \in \mathbb{K}[t]$ denote the monic least common multiple of the denominators of $\mathbf{f}$, which is well-defined by (2.2.4.1). Then since $\boldsymbol{\sigma} \mu=\mu$, we have

$$
\mathbf{f} \cdot \mathbf{p}=\boldsymbol{\sigma}(\mathbf{f} \cdot \mathbf{p})=\mathbf{f}^{(-1)} \cdot \Phi \mathbf{p} .
$$

Therefore, $\operatorname{den}(\mathbf{f})=\operatorname{den}\left(\mathbf{f}^{(-1)} \cdot \Phi\right)$. But $\Phi \in \operatorname{Mat}_{r}(\bar{k}[t])$, so $\operatorname{den}\left(\mathbf{f}^{(-1)} \cdot \Phi\right)$ divides $\operatorname{den}\left(\mathbf{f}^{(-1)}\right)$. Degree considerations force $\operatorname{den}(\mathbf{f})=\operatorname{den}\left(\mathbf{f}^{(-1)}\right)$. Therefore take $c=$ $\operatorname{den}(\mathbf{f}) \in \mathbb{F}_{q}[t]$. This proves the claim, and moreover we have shown that

$$
P^{\mathrm{B}} \cong \mathbb{F}_{q}(t) \otimes_{\mathbb{F}_{q}[t]} \mathrm{P}^{\mathrm{B}}
$$

Furthermore, it follows that as $\mathbb{L}$-vector spaces, $P^{\dagger} \cong \mathbb{L} \otimes_{\mathbb{T}} \mathrm{P}^{\dagger} \cong \mathbb{L} \otimes_{\mathbb{F}_{q}[t]} \mathrm{P}^{\mathrm{B}}$. Let $\boldsymbol{\nu}=$ $\left[\nu_{1}, \ldots, \nu_{r}\right]^{\text {tr }}$ be an $\mathbb{F}_{q}[t]$-basis for $\mathrm{P}^{\mathrm{B}}$. Then for some $\Theta \in \mathrm{GL}_{r}(\mathbb{L}) \cap \operatorname{Mat}_{r}(\mathbb{T})$, we have $\boldsymbol{\nu}=\Theta \mathbf{p}$. Since $\boldsymbol{\sigma} \boldsymbol{\nu}=\boldsymbol{\nu}$, it follows that $\Theta^{(-1)} \Phi=\Theta$. By our initial hypotheses, $d(t-$ $\theta)^{s} \operatorname{det}(\Theta)^{(-1)}=\operatorname{det}(\Theta)$. Choose $b \in \bar{k}^{\times}$so that $d=b^{(-1)} / b$. Then from Lemmas 3.3 .2 and 3.3 .5 (and the fact that $\Theta \in \operatorname{Mat}_{r}(\mathbb{T})$ ), we see that

$$
b \operatorname{det}(\Theta)=\frac{\gamma}{\Omega^{s}}, \quad \gamma \in \mathbb{F}_{q}[t] .
$$

We claim that $\gamma \in \mathbb{F}_{q}^{\times}$. If not, then $\operatorname{det}(\Theta) \equiv 0(\bmod \gamma)$ in $\mathbb{T}$, and so there is a $\mathbf{f}=\left[f_{1}, \ldots, f_{r}\right] \in \operatorname{Mat}_{1 \times r}(\mathbb{T})$ so that

$$
\mathbf{f} \cdot \Theta \equiv 0 \quad(\bmod \gamma)
$$

Since $\mathbb{T} / \gamma \mathbb{T} \cong \mathbb{K}[t] / \gamma \mathbb{K}[t]$, without loss of generality we can assume that each $f_{i}$ is a polynomial in $\mathbb{K}[t]$ of degree strictly less than the degree of $\gamma$, that $\left\|f_{i}\right\| \leq 1$ for all $i$, and that at least one $f_{i}$ satisfies $\left\|f_{i}\right\|=1$. Now define a norm $\|\cdot\|_{\dagger}$ on $\mathrm{P}^{\dagger}$ by

$$
\left\|\sum h_{i} p_{i}\right\|_{\dagger}:=\sup \left\|h_{i}\right\|, \quad h_{1}, \ldots, h_{r} \in \mathbb{T} .
$$

Then $\|\cdot\|_{\dagger}$ defines a complete ultrametic norm on $\mathrm{P}^{\dagger}$ that satisfies

$$
\|h \mu\|_{\dagger}=\|h\| \cdot\|\mu\|_{\dagger}, \quad h \in \mathbb{T}, \mu \in \mathrm{P}^{\dagger} .
$$

Consider

$$
\mathbf{f} \cdot \Theta^{(-1)} \Phi=\mathbf{f} \cdot \Theta \equiv 0 \quad(\bmod \gamma) .
$$

Since $\gamma$ is relatively prime to $\operatorname{det}(\Phi)$, it follows that $\Phi$ is invertible modulo $\gamma$, and so

$$
\mathbf{f} \cdot \Theta^{(-1)} \equiv 0 \quad(\bmod \gamma) .
$$

Repeating this argument we find that $\mathbf{f} \cdot \Theta^{(-n)} \equiv 0(\bmod \gamma)$ for all $n \geq 0$. Now, by choice of $\mathbf{f}$,

$$
\frac{1}{\gamma} \mathbf{f} \cdot \boldsymbol{\nu}=\frac{1}{\gamma} \mathbf{f} \cdot \Theta \cdot \mathbf{p} \in \mathbf{P}^{\dagger},
$$

and for each $n$, the above congruences for $\mathbf{f} \cdot \Theta^{(-n)}$ imply that $\gamma^{-1} \mathbf{f}^{(n)} \cdot \boldsymbol{\nu}=\gamma^{-1} \mathbf{f}^{(n)} \cdot \Theta \cdot \mathbf{p} \in$ $\mathrm{P}^{\dagger}$. Now by Lemma 2.2.7 there is an $m>0$ so that with respect to the $\|\cdot\|_{\dagger}$ metric,

$$
\lim _{n \rightarrow \infty} \frac{1}{\gamma} \sum f_{i}^{(m n)} \nu_{i}=\frac{1}{\gamma} \sum c_{i} \nu_{i} \in \mathrm{P}^{\dagger},
$$

where $c_{i} \in \overline{\mathbb{F}}_{q}[t]$ and at least one $c_{i} \neq 0$, say $c_{a} \neq 0$. Now for some $l \geq 1$, we have every $c_{i} \in \mathbb{F}_{q^{l}}[t]$. Since the trace map $\mathbb{F}_{q^{l}} \rightarrow \mathbb{F}_{q}$ is not trivial, by dividing each $c_{i}$ by a fixed 
element in $\mathbb{F}_{q^{l}}^{\times}$, we can assume that $c_{a}+c_{a}^{(-1)}+\cdots+c_{a}^{(1-l)} \neq 0$. Therefore,

$$
\sum_{j=0}^{l-1} \boldsymbol{\sigma}^{j}\left(\frac{1}{\gamma} \sum_{i=1}^{r} c_{i} \nu_{i}\right)=\frac{1}{\gamma} \sum_{i=1}^{r}\left(\sum_{j=0}^{l-1} c_{i}^{(-j)}\right) \nu_{i} \in \mathrm{P}^{\dagger}
$$

Thus we obtain $\mu:=\gamma^{-1} \sum d_{i} \nu_{i} \in \mathrm{P}^{\dagger}, d_{i} \in \mathbb{F}_{q}[t], d_{a} \neq 0$. Easily we see that $\mu \in \mathrm{P}^{\mathrm{B}}$ and $\mu \neq 0$. Since $\operatorname{deg} f_{i}<\operatorname{deg} \gamma$ for each $i$, we have $\operatorname{deg} d_{i}<\operatorname{deg} \gamma$ for each $i$. In particular, $\gamma$ does not divide $d_{a}$. Thus $\mu \in \mathrm{P}^{\mathrm{B}}$ but $\mu$ is not in the $\mathbb{F}_{q}[t]$-span of $\boldsymbol{\nu}$, which contradicts that $\boldsymbol{\nu}$ is an $\mathbb{F}_{q}[t]$-basis of $\mathrm{P}^{\mathrm{B}}$. Therefore, it follows that $\gamma \in \mathbb{F}_{q}^{\times}$, and since $\Omega \in \mathbb{T}^{\times}$, we have $\operatorname{det}(\Theta) \in \mathbb{T}^{\times}$. Taking $\Psi=\Theta^{-1}$ provides the desired rigid analytic trivialization.

3.3.10. Remark. It is worth noting that multiplication by $\Theta$ induces the isomorphism of $\mathbb{L}$-vector spaces,

$$
\mathbb{L} \otimes_{\mathbb{T}} \mathrm{P}^{\dagger} \cong \mathbb{L} \otimes_{\mathbb{T}}\left(\mathbb{T} \otimes_{\mathbb{F}_{q}[t]} \mathrm{P}^{\mathrm{B}}\right) .
$$

Since $\Theta \in \mathrm{GL}_{r}(\mathbb{T})$, this then implies $\mathrm{P}^{\dagger} \cong \mathbb{T} \otimes_{\mathbb{F}_{q}[t]} \mathrm{P}^{\mathrm{B}}$ as $\mathbb{T}$-modules.

Proposition 3.3.11. Let

$$
0 \rightarrow P \rightarrow Q \rightarrow R \rightarrow 0
$$

be an exact sequence of pre-t-motives.

(a) If $Q$ is rigid analytically trivial, then both $P$ and $R$ are rigid analytically trivial.

(b) If $P, Q$, and $R$ are rigid analytically trivial, then the sequence

$$
0 \rightarrow P^{\mathrm{B}} \rightarrow Q^{\mathrm{B}} \rightarrow R^{\mathrm{B}} \rightarrow 0
$$

is an exact sequence of $\mathbb{F}_{q}(t)$-vector spaces.

Proof. The sequence $0 \rightarrow P^{\mathrm{B}} \rightarrow Q^{\mathrm{B}} \rightarrow R^{\mathrm{B}}$ is exact. Now suppose that $Q$ is rigid analytically trivial. Let $\kappa: \mathbb{L} \otimes_{\mathbb{F}_{q}(t)} Q^{\mathrm{B}} \rightarrow \mathbb{L} \otimes_{\mathbb{F}_{q}(t)} R^{\mathrm{B}}$ be the natural map. Then we have a commutative diagram with exact rows,

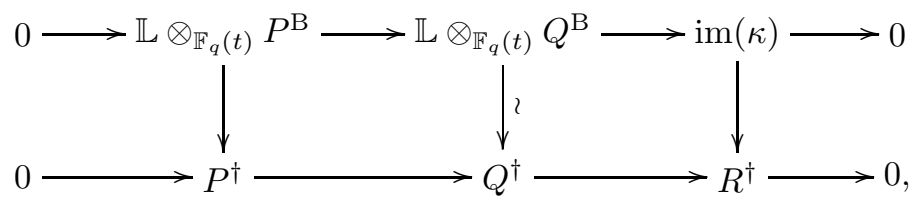

where the central vertical map is an isomorphism by hypothesis, and the other two are injective by Lemma 3.3.7. The injectivity of all three maps then implies that each is an isomorphism. Thus we see immediately that $P$ is rigid analytically trivial. Also we see that

$$
\operatorname{dim}_{\mathbb{F}_{q}(t)} R^{\mathrm{B}}=\operatorname{dim}_{\mathbb{L}} \mathbb{L} \otimes_{\mathbb{F}_{q}(t)} R^{\mathrm{B}} \geq \operatorname{dim}_{\mathbb{L}} \operatorname{im}(\kappa)=\operatorname{dim}_{\mathbb{L}} R^{\dagger}=\operatorname{dim}_{\bar{k}(t)} R,
$$

which by Proposition 3.3 .8 must be a string of equalities. Therefore $R$ is rigid analytically trivial, which completes part (a).

Now suppose that $P, Q$, and $R$ are all rigid analytically trivial. Then

$$
\operatorname{dim}_{\mathbb{F}_{q}(t)} Q^{\mathrm{B}}=\operatorname{dim}_{\bar{k}(t)} Q=\operatorname{dim}_{\bar{k}(t)} P+\operatorname{dim}_{\bar{k}(t)} R=\operatorname{dim}_{\mathbb{F}_{q}(t)} P^{\mathrm{B}}+\operatorname{dim}_{\mathbb{F}_{q}(t)} R^{\mathrm{B}},
$$

which proves part (b). 
3.3.12. Remark. In particular, it follows from Proposition 3.3.11 that kernels and cokernels exist in $\mathcal{R}$, which implies that $\mathcal{R}$ is an abelian $\mathbb{F}_{q}(t)$-linear category. We also see that

$$
P \rightarrow P^{\mathrm{B}}: \mathcal{R} \rightarrow \operatorname{Vec}\left(\mathbb{F}_{q}(t)\right)
$$

where $\operatorname{Vec}\left(\mathbb{F}_{q}(t)\right)$ is the category of finite dimensional vector spaces over $\mathbb{F}_{q}(t)$, is an exact $\mathbb{F}_{q}(t)$-linear functor.

Proposition 3.3.13. Let $P$ and $Q$ be rigid analytically trivial pre-t-motives. Then the natural map

$$
\operatorname{Hom}_{\mathcal{R}}(P, Q) \rightarrow \operatorname{Hom}_{\mathbb{F}_{q}(t)}\left(P^{\mathrm{B}}, Q^{\mathrm{B}}\right)
$$

is injective.

Proof. Suppose $\phi: P \rightarrow Q$ is a morphism in $\operatorname{Hom}_{\mathcal{R}}(P, Q)$. Then we have an exact sequence in $\mathcal{R}$,

$$
0 \rightarrow \operatorname{ker} \phi \rightarrow P \stackrel{\phi}{\rightarrow} Q \rightarrow Q / \phi(P) \rightarrow 0,
$$

which leads then to an exact sequence of $\mathbb{F}_{q}(t)$-vector spaces,

$$
0 \rightarrow(\operatorname{ker} \phi)^{\mathrm{B}} \rightarrow P^{\mathrm{B}} \stackrel{\phi^{\mathrm{B}}}{\rightarrow} Q^{\mathrm{B}} \rightarrow(Q / \phi(P))^{\mathrm{B}} \rightarrow 0 .
$$

Since the dimension over $\bar{k}(t)$ of each term in the first sequence is the same as the dimension over $\mathbb{F}_{q}(t)$ of the corresponding term in the second sequence, we see that $\phi^{\mathrm{B}}=0$ if and only if $\phi=0$.

Proposition 3.3.14. If pre-t-motives $P$ and $Q$ are rigid analytically trivial, then

(a) $P \otimes Q$ is rigid analytically trivial, and the natural map $P^{\mathrm{B}} \otimes_{\mathbb{F}_{q}(t)} Q^{\mathrm{B}} \rightarrow(P \otimes Q)^{\mathrm{B}}$ is an isomorphism of $\mathbb{F}_{q}(t)$-vector spaces;

(b) $P^{\vee}$ is rigid analytically trivial, and the natural map $\left(P^{\mathrm{B}}\right)^{\vee} \rightarrow\left(P^{\vee}\right)^{\mathrm{B}}$ is an isomorphism of $\mathbb{F}_{q}(t)$-vector spaces.

Proof. Here we make use of Proposition 3.3.9. We first note that

$$
(P \otimes Q)^{\dagger}=\mathbb{L} \otimes_{\bar{k}(t)}\left(P \otimes_{\bar{k}(t)} Q\right) \cong\left(\mathbb{L} \otimes_{\bar{k}(t)} P\right) \otimes_{\mathbb{L}}\left(\mathbb{L} \otimes_{\bar{k}(t)} Q\right)=P^{\dagger} \otimes_{\mathbb{L}} Q^{\dagger},
$$

where the middle isomorphism is an isomorphism of $\mathbb{L}$-vector spaces that commutes with the action of $\boldsymbol{\sigma}$. We observe that we can choose $\bar{k}(t)$-bases for $P, Q$, and $P \otimes Q$ so that multiplication by $\boldsymbol{\sigma}$ is represented by matrices $\Phi_{P}, \Phi_{Q}$, and $\Phi_{P \otimes Q}$ satisfying

$$
\Phi_{P \otimes Q}=\Phi_{P} \otimes \Phi_{Q}
$$

By Proposition 3.3.9 we can choose $\Psi_{P}, \Psi_{Q} \in \mathrm{GL}_{r}(\mathbb{L})$ that are rigid analytic trivializations of $\Phi_{P}$ and $\Phi_{Q}$. Then we note that $\Psi_{P \otimes Q}:=\Psi_{P} \otimes \Psi_{Q}$ is a rigid analytic trivialization of $\Phi_{P \otimes Q}$. Now note that $\Phi_{P^{\vee}}:=\left(\Phi_{P}^{-1}\right)^{\operatorname{tr}}$ represents multiplication by $\boldsymbol{\sigma}$ with respect to the dual basis and that $\Psi_{P}^{\vee}:=\left(\Psi_{P}^{-1}\right)^{\text {tr }}$ is a rigid analytic trivialization. The second parts of (a) and (b) are straightforward.

Theorem 3.3.15. The category $\mathcal{R}$ of rigid analytically trivial pre-t-motives is a neutral Tannakian category over $\mathbb{F}_{q}(t)$ with fiber functor $P \mapsto P^{\mathrm{B}}: \mathcal{R} \rightarrow \operatorname{Vec}\left(\mathbb{F}_{q}(t)\right)$.

Proof. We have seen that

- 1 is in $\mathcal{R}$ (Proposition 3.3.3);

- $\mathcal{R}$ is an abelian category (Proposition 3.3.11 and \$3.3.12);

- $\mathcal{R}$ is closed under tensor products and duals (Proposition 3.3.14).

Thus $\mathcal{R}$ is a rigid abelian $\mathbb{F}_{q}(t)$-linear tensor subcategory of $\mathcal{P}$ (see [11, Defs. II.1.14-15]). We have also shown that 
- $\operatorname{End}_{\mathcal{R}}(\mathbf{1})=\mathbb{F}_{q}(t)($ Lemma 3.2.9) ;

- For each $P$ in $\mathcal{R}$, the $\mathbb{F}_{q}(t)$-vector space $P^{\mathrm{B}}$ is finite dimensional (Proposition 3.3.8);

- $P \mapsto P^{\mathrm{B}}$ is $\mathbb{F}_{q}(t)$-linear and exact (Proposition 3.3.11 and \$3.3.12);

- $P \mapsto P^{\mathrm{B}}$ is faithful (Proposition 3.3.13);

- $P \mapsto P^{\mathrm{B}}$ is a tensor functor (Proposition 3.3.14).

Thus $\mathcal{R}$ is a neutral Tannakian category over $\mathbb{F}_{q}(t)$ with fiber functor $P \mapsto P^{\mathrm{B}}$ (see [11, Def. II.2.19]).

3.4. Anderson $t$-motives. Here we recall the definitions and essential properties of "dual $t$-motives" from [2. So as not to confuse these objects with the duals of $t$-motives to be used later on, we call these objects Anderson t-motives, since they are simply the dual notion of the objects studied in 1 .

3.4.1. Definition. An Anderson $t$-motive $\mathrm{M}$ is a left $\bar{k}[t ; \boldsymbol{\sigma}]$-module such that

- $\mathrm{M}$ is free and finitely generated over $\bar{k}[t]$;

- $\mathrm{M}$ is free and finitely generated over $\bar{k}[\boldsymbol{\sigma}]$;

- $(t-\theta)^{n} \mathrm{M} \subseteq \boldsymbol{\sigma} \mathrm{M}$ for all $n \gg 0$.

A morphism of Anderson $t$-motives is a left $\bar{k}[t ; \boldsymbol{\sigma}]$-module homomorphism. In this way Anderson $t$-motives form a category.

As in $\$ 3.2 .3$, if $\mathrm{m} \in \mathrm{Mat}_{r \times 1}(\mathrm{M})$ is a $\bar{k}[t]$-module basis for $\mathrm{M}$, then there is a matrix $\Phi=\Phi_{\mathrm{m}} \in \operatorname{Mat}_{r \times 1}(\bar{k}[t])$ so that

$$
\sigma \mathrm{m}=\Phi \mathrm{m} .
$$

Since a power of $t-\theta$ annihilates $\mathrm{M} / \sigma \mathrm{M}$, we have

$$
\operatorname{det} \Phi=c(t-\theta)^{s}
$$

for some $c \in \bar{k}^{\times}$, where $s$ is the rank of $\mathrm{M}$ as a $\bar{k}[\boldsymbol{\sigma}]$-module.

3.4.2. Anderson t-motives to pre-t-motives. Given an Anderson $t$-motive $\mathrm{M}$ we obtain a pre-t-motive $M$ by setting

$$
M:=\bar{k}(t) \otimes_{\bar{k}[t]} \mathrm{M}
$$

and defining

$$
\boldsymbol{\sigma}(f \otimes m):=f^{(-1)} \otimes \boldsymbol{\sigma} m .
$$

It is straightforward to check that $M$ is a left $\bar{k}(t)\left[\boldsymbol{\sigma}, \boldsymbol{\sigma}^{-1}\right]$-module, and it is of course finite dimensional as a $\bar{k}(t)$-vector space. Moreover, $\mathrm{M} \mapsto M$ is a functor from the category of Anderson $t$-motives to the category of pre- $t$-motives.

3.4.3. The Carlitz motive. Let $\mathrm{C}$ be the Anderson $t$-motive whose underlying $\bar{k}[t]$ module is $\bar{k}[t]$ itself. Then the action of $\boldsymbol{\sigma}$ on $\mathrm{C}$ is defined by

$$
\boldsymbol{\sigma}(f)=(t-\theta) f^{(-1)}, \quad f \in \mathrm{C} .
$$

It is not difficult to check that $C$ is an Anderson $t$-motive, and that its image in $\mathcal{P}$ is the Carlitz motive. For any $n \geq 1$, we also have the $n$-th tensor power of $\mathrm{C}$,

$$
\mathrm{C}(n):=\mathrm{C} \otimes_{\bar{k}[t]} \cdots \otimes_{\bar{k}[t]} \mathrm{C},
$$

with diagonal $\boldsymbol{\sigma}$-action. It is an Anderson $t$-motive sent to $C(n)$ in $\mathcal{P}$. 
3.4.4. The Carlitz module. The Carlitz module $\mathfrak{C}$ over $\bar{k}$ is defined to be the $\mathbb{F}_{q}$-algebra $\bar{k}$ together with an $\mathbb{F}_{q}[t]$-module structure defined by

$$
\mathfrak{C}_{t}(x):=\theta x+x^{q}, \quad x \in \bar{k} .
$$

That is the $\mathbb{F}_{q^{-}}$algebra homomorphism $a \mapsto \mathfrak{C}_{a}: \mathbb{F}_{q}[t] \rightarrow \bar{k}\left[\boldsymbol{\sigma}^{-1}\right]$ defined by $t \mapsto \theta+\boldsymbol{\sigma}^{-1}$ induces an $\mathbb{F}_{q}[t]$-module structure on $\bar{k}$. See [15, Ch. 3] or [31, §2.5] for more details. To see the relationship with the Carlitz motive, we note that there is an isomorphism

$$
\mathfrak{C}(\bar{k}) \cong \frac{\mathrm{C}}{(\boldsymbol{\sigma}-1) \mathrm{C}}
$$

of $\mathbb{F}_{q}[t]$-modules. Indeed if $x \in \bar{k}$, then

$$
t x=\theta x+(t-\theta) x=\theta x+\boldsymbol{\sigma}\left(x^{q}\right)=\theta x+x^{q}+(\boldsymbol{\sigma}-1) x^{q} .
$$

Similarly $a x \equiv \mathfrak{C}_{a}(x)(\bmod \sigma-1)$ for all $a \in \mathbb{F}_{q}[t]$. It is a simple matter to check that there is a natural isomorphism of $\mathbb{F}_{q}$-vector spaces $\mathrm{C} /(\boldsymbol{\sigma}-1) \mathrm{C} \cong \bar{k}$. Thus $\mathrm{C} /(\boldsymbol{\sigma}-1) \mathrm{C}$ presents the Carlitz module directly.

Proposition 3.4.5. For Anderson t-motives $\mathrm{M}$ and $\mathrm{N}$, the natural map

$$
\operatorname{Hom}_{\bar{k}[t ; \sigma]}(\mathrm{M}, \mathrm{N}) \otimes_{\mathbb{F}_{q}[t]} \mathbb{F}_{q}(t) \rightarrow \operatorname{Hom}_{\mathcal{P}}(M, N)
$$

is an isomorphism of $\mathbb{F}_{q}(t)$-vector spaces.

Proof. Let $\Theta$ denote the map in question. It is clearly $\mathbb{F}_{q}(t)$-linear. To see that it is injective, we first observe that if $\alpha \in \operatorname{Hom}_{\bar{k}[t ; \sigma]}(\mathrm{M}, \mathrm{N}) \otimes_{\mathbb{F}_{q}[t]} \mathbb{F}_{q}(t)$ then $\alpha=\underline{\phi} \otimes \frac{1}{v}$, for some $\underline{\phi} \in \operatorname{Hom}_{\bar{k}[t ; \sigma]}(\mathrm{M}, \mathrm{N})$ and $v \in \mathbb{F}_{q}[t], v \neq 0$. Then $v \Theta(\alpha)=\Theta(v \alpha)=\Theta(\underline{\phi} \otimes 1)=: \phi$. But

$$
\begin{aligned}
& \underline{\phi} \in \operatorname{Hom}_{\bar{k}[t ; \boldsymbol{\sigma}]}(\mathrm{M}, \mathrm{N}) \subseteq \operatorname{Hom}_{\bar{k}[t]}(\mathrm{M}, \mathrm{N}), \\
& \phi \in \operatorname{Hom}_{\bar{k}(t)\left[\boldsymbol{\sigma}, \boldsymbol{\sigma}^{-1}\right]}(M, N) \subseteq \operatorname{Hom}_{\bar{k}(t)}(M, N),
\end{aligned}
$$

and so $\phi=0$ if and only if $\underline{\phi}=0$. Thus $\Theta(\alpha)=0$ if and only if $\alpha=0$.

For surjectivity, suppose that $\phi \in \operatorname{Hom}_{\mathcal{P}}(M, N)$. Fix $\bar{k}[t]$-bases $\mathrm{m}$ and $\mathrm{n}$ for $\mathrm{M}$ and $\mathrm{N}$ respectively, and extend these to bases $\mathbf{m}$ and $\mathbf{n}$ of $M$ and $N$. Then the map $\phi: M \rightarrow N$ is represented by a matrix $F \in \operatorname{Mat}_{r \times s}(\bar{k}(t))$ so that $F^{(-1)} \Phi_{\mathbf{n}}=\Phi_{\mathbf{m}} F$ as in 3.2 .3 . By choice of $\mathrm{m}$ and $\mathrm{n}, \Phi_{\mathbf{m}}$ and $\Phi_{\mathbf{n}}$ have entries in $\bar{k}[t]$, and it suffices to show that $F$ has entries with denominators in $\mathbb{F}_{q}[t]$.

For a matrix $B$ with entries in $\bar{k}(t)$, let $\operatorname{den}(B) \in \bar{k}[t]$ be the monic least common multiple of the denominators of the entries of $B$. Since $\operatorname{det}\left(\Phi_{\mathbf{n}}\right)=c(t-\theta)^{s}$ for some $s \geq 0$ and $c \in \bar{k}^{\times}$, we see that

$$
\operatorname{den}(F)(t-\theta)^{s} \cdot F^{(-1)}=\operatorname{den}(F)(t-\theta)^{s} \cdot \Phi_{\mathbf{m}} F \Phi_{\mathbf{n}}^{-1} \in \operatorname{Mat}_{r \times s}(\bar{k}[t]) .
$$

Therefore, $\operatorname{den}\left(F^{(-1)}\right)$ divides $\operatorname{den}(F)(t-\theta)^{s}$. However, $\operatorname{den}\left(F^{(-1)}\right)=\operatorname{den}(F)^{(-1)}$ and so $\operatorname{deg}\left(\operatorname{den}\left(F^{(-1)}\right)\right)=\operatorname{deg}(\operatorname{den}(F))$. Thus, it suffices to show that $\operatorname{den}\left(F^{(-1)}\right)$ is relatively prime to $t-\theta$, since then $\operatorname{den}(F)^{(-1)}=\operatorname{den}(F)$ whence all of the denominators of $F$ are in $\mathbb{F}_{q}[t]$.

Suppose that $t-\theta$ divides $\operatorname{den}\left(F^{(-1)}\right)$, and so $t-\theta^{q} \operatorname{divides} \operatorname{den}(F)$. Then $t-\theta^{q}$ divides $\operatorname{den}\left(\Phi_{\mathbf{m}} F\right)$, because otherwise $t-\theta^{q}$ would $\operatorname{divide} \operatorname{det}\left(\Phi_{\mathbf{m}}\right)$ which is a power of $t-\theta$. Likewise, $t-\theta^{q}$ divides $\operatorname{den}\left(\Phi_{\mathbf{m}} F \Phi_{\mathbf{n}}^{-1}\right)=\operatorname{den}\left(F^{(-1)}\right)$. By repeating the same argument we see that $\operatorname{den}\left(F^{(-1)}\right)$ is divisible by each of

$$
t-\theta, t-\theta^{q}, t-\theta^{q^{2}}, \ldots
$$

contradicting that $\operatorname{den}\left(F^{(-1)}\right) \in \bar{k}[t]$. 
3.4.6. Rigid analytic triviality. Similar to 33.3 .1 if $\mathrm{M}$ is an Anderson $t$-motive, then we set

$$
\mathrm{M}^{\dagger}:=\mathbb{T} \otimes_{\bar{k}[t]} \mathrm{M}
$$

We provide $\mathrm{M}^{\dagger}$ with a $\bar{k}[t ; \boldsymbol{\sigma}]$-module structure by setting $\boldsymbol{\sigma}(f \otimes m)=f^{(-1)} \otimes \boldsymbol{\sigma} m$, and we set

$$
\mathrm{M}^{\mathrm{B}}:=\left(\mathrm{M}^{\dagger}\right)^{\sigma}=\left\{\mu \in \mathrm{M}^{\dagger} \mid \sigma \mu=\mu\right\}
$$

We say that $\mathrm{M}$ is rigid analytically trivial if the natural map $\mathbb{T} \otimes_{\mathbb{F}_{q}[t]} \mathrm{M}^{\mathrm{B}} \rightarrow \mathrm{M}^{\dagger}$ is an isomorphism. The following proposition is a companion to Proposition 3.3 .9

Proposition 3.4.7. Let $\mathrm{M}$ be an Anderson t-motive, and let $M$ be its corresponding pre-t-motive. Suppose $\mathrm{m} \in \mathrm{Mat}_{r \times 1}(\mathrm{M})$ is a $\bar{k}[t]$-basis for $\mathrm{M}$, and let $\Phi \in \operatorname{Mat}_{r \times 1}(\bar{k}[t])$ represent multiplication by $\boldsymbol{\sigma}$ on $\mathrm{M}$ with respect to $\mathrm{m}$.

(a) $\mathrm{M}$ is rigid analytically trivial if and only if it admits a rigid analytic trivialization $\Psi$ with $\Psi \in \mathrm{GL}_{r}(\mathbb{T})$.

(b) If $\Psi \in \mathrm{GL}_{r}(\mathbb{T})$ is a rigid analytic trivialization of $\Phi$, then the entries of $\Psi^{-1} \mathrm{~m}$ form an $\mathbb{F}_{q}[t]$-basis of $\mathrm{M}^{\mathrm{B}}$.

(c) $\mathrm{M}$ is rigid analytically trivial if and only if $M$ is rigid analytically trivial.

Proof. The proofs of parts (a) and (b) are in [2, Lem. 4.4.13] and follow the same lines as their counterparts in Proposition 3.3.9. Part (c) is then a consequence of Proposition 3.3.9. (c).

3.4.8. Definition. We define the category $\mathcal{A}^{I}$ of Anderson t-motives up to isogeny as follows:

- Objects of $\mathcal{A}^{I}$ : Anderson $t$-motives;

- Morphisms of $\mathcal{A}^{I}$ : For Anderson $t$-motives $\mathrm{M}$ and $\mathrm{N}$,

$$
\operatorname{Hom}_{\mathcal{A}^{I}}(\mathrm{M}, \mathrm{N}):=\operatorname{Hom}_{\bar{k}[t ; \boldsymbol{\sigma}]}(\mathrm{M}, \mathrm{N}) \otimes_{\mathbb{F}_{q}[t]} \mathbb{F}_{q}(t) .
$$

We also define the full subcategory $\mathcal{A} \mathcal{R}^{I}$ of rigid analytically trivial Anderson $t$-motives up to isogeny by restriction. We sum up the results of this section in the following theorem.

Theorem 3.4.9. Let $\mathcal{P}$ be the category of pre-t-motives, and let $\mathcal{R}$ be the category of rigid analytically trivial pre-t-motives.

(a) The functor $\mathrm{M} \mapsto M: \mathcal{A}^{I} \rightarrow \mathcal{P}$ is fully faithful.

(b) The functor $\mathrm{M} \mapsto M: \mathcal{A R} \mathcal{R}^{I} \rightarrow \mathcal{R}$ is fully faithful.

Proof. Part (a) is simply a restatement of Proposition 3.4.5. That the functor in part (b) is well-defined follows from Proposition 3.4 .7 (c), and its full faithfulness follows from Proposition 3.4.5

3.4.10. The category $\mathcal{T}$. We define the category $\mathcal{T}$ of $t$-motives to be the strictly full Tannakian subcategory of $\mathcal{R}$ generated by the essential image of the functor

$$
\mathrm{M} \mapsto M: \mathcal{A R} \mathcal{R}^{I} \rightarrow \mathcal{R} .
$$

The category of $t$-motives can further be described as follows:

- Objects of $\mathcal{T}$ : rigid analytically trivial pre- $t$-motives that can be constructed from Anderson $t$-motives using direct sums, subquotients, tensor products, duals, and internal Hom's.

- Morphisms of $\mathcal{T}$ : morphisms of left $\bar{k}(t)\left[\boldsymbol{\sigma}, \boldsymbol{\sigma}^{-1}\right]$-modules.

It is worth noting that Proposition 3.4.7(c) says that the category of $t$-motives is the strictly full Tannakian subcategory of $\mathcal{R}$ generated by the intersection in $\mathcal{P}$ of $\mathcal{R}$ and the image of all Anderson $t$-motives. 
3.5. Galois groups of $t$-motives. Having defined a Tannakian category of $t$-motives, it is now possible to assign to each $t$-motive a linear algebraic group over $\mathbb{F}_{q}(t)$, which we call the Galois group of the $t$-motive. For essential facts about Tannakian categories and their associated groups, we refer to [8], [11, 26, App. B].

3.5.1. Fiber functors. The functor

$$
\begin{aligned}
\omega: \mathcal{T} & \rightarrow \operatorname{Vec}\left(\mathbb{F}_{q}(t)\right) \\
M & \mapsto M^{\mathrm{B}}
\end{aligned}
$$

is the fiber functor of $\mathcal{T}$. For any commutative $\mathbb{F}_{q}(t)$-algebra $R$, we let $\omega^{(R)}: \mathcal{T} \rightarrow$ $\operatorname{Mod}(R)$ be the extension of $\omega$ defined by

$$
\omega^{(R)}(M):=R \otimes_{\mathbb{F}_{q}(t)} M^{\mathrm{B}},
$$

where $\operatorname{Mod}(R)$ is the category of finitely generated left $R$-modules. Now fix a $t$-motive $M$. We let $\mathcal{T}_{M}$ be the strictly full Tannakian subcategory of $\mathcal{T}$ generated by $M$. That is, $\mathcal{T}_{M}$ consists of all objects of $\mathcal{T}$ isomorphic to subquotients of finite direct sums of $M^{\otimes u} \otimes\left(M^{\vee}\right)^{\otimes v}$ for various $u, v$. The fiber functor of $\mathcal{T}_{M}$ is $\omega_{M}: \mathcal{T}_{M} \rightarrow \operatorname{Vec}\left(\mathbb{F}_{q}(t)\right)$, the restriction of $\omega$ to $\mathcal{T}_{M}$, and similarly we restrict $\omega_{M}^{(R)}$ to $\mathcal{T}_{M}$ for an $\mathbb{F}_{q}(t)$-algebra $R$.

3.5.2. Galois groups. As $\mathcal{T}$ is a neutral Tannakian category over $\mathbb{F}_{q}(t)$, there is an affine group scheme $\Gamma_{\mathcal{T}}$ over $\mathbb{F}_{q}(t)$ so that $\mathcal{T}$ is equivalent to the category $\operatorname{Rep}\left(\Gamma_{\mathcal{T}}, \mathbb{F}_{q}(t)\right)$ of finite dimensional representations of $\Gamma_{\mathcal{T}}$ over $\mathbb{F}_{q}(t)$ :

$$
\mathcal{T} \approx \operatorname{Rep}\left(\Gamma_{\mathcal{T}}, \mathbb{F}_{q}(t)\right) .
$$

The group $\Gamma_{\mathcal{T}}$ is defined to be the group of tensor automorphisms of the fiber functor $\omega$; that is, if $R$ is any $\mathbb{F}_{q}(t)$-algebra, then

$$
\Gamma_{\mathcal{T}}(R)=\operatorname{Aut}_{\mathcal{T}}^{\otimes}\left(\omega^{(R)}\right) .
$$

Now for any $t$-motive $M$, there is a linear algebraic group $\Gamma_{M}:=\Gamma_{\mathcal{T}_{M}}$ over $\mathbb{F}_{q}(t)$ so that $\mathcal{T}_{M}$ is equivalent to $\operatorname{Rep}\left(\Gamma_{M}, \mathbb{F}_{q}(t)\right)$. As such, for any $\mathbb{F}_{q}(t)$-algebra $R, \Gamma_{M}(R)=$ $\operatorname{Aut}_{\mathcal{T}_{M}}^{\otimes}\left(\omega_{M}^{(R)}\right)$. In this way we find that we have a naturally defined faithful representation

$$
\Gamma_{M} \hookrightarrow \mathrm{GL}\left(M^{\mathrm{B}}\right)
$$

over $\mathbb{F}_{q}(t)$, which provides the basis for constructing the equivalence of categories,

$$
\mathcal{T}_{M} \approx \operatorname{Rep}\left(\Gamma_{M}, \mathbb{F}_{q}(t)\right) .
$$

The group $\Gamma_{M}$ is called the Galois group of $M$. Furthermore, there is a surjective group homomorphism,

$$
\Gamma_{\mathcal{T}} \rightarrow \Gamma_{M} .
$$

If $N$ is another $t$-motive in $\mathcal{T}_{M}$, then there is a natural surjective homomorphism, $\Gamma_{M} \rightarrow \Gamma_{N}$. In 44 , we will show that $\Gamma_{M}$ can be calculated using systems of $\sigma$-semilinear equations. For now we will calculate the Galois group of the Carlitz motive $C$.

Lemma 3.5.3. For $m, n \in \mathbb{Z}$,

$$
\operatorname{Hom}_{\mathcal{T}}(C(m), C(n)) \cong \begin{cases}\mathbb{F}_{q}(t) & \text { if } m=n, \\ 0 & \text { if } m \neq n .\end{cases}
$$

Proof. By tensoring with $C(-m)$, we see that $\operatorname{Hom}_{\mathcal{T}}(C(m), C(n)) \cong \operatorname{Hom}_{\mathcal{T}}(\mathbf{1}, C(n-$ $m)$ ). Thus it suffices to assume that $m=0$. If $\phi: \mathbf{1} \rightarrow C(n)$ is a morphism in $\mathcal{T}$, then $\phi$ is represented by some $a \in \bar{k}(t)^{\times}$such that $a=a^{(-1)}(t-\theta)^{n}$. By Lemma 3.3.5, this equation has no non-zero solutions $a \in \bar{k}(t)$ unless $n=0$, in which case $a$ can be anything in $\mathbb{F}_{q}(t)$. 
Theorem 3.5.4. For the Carlitz motive $C$, there is an isomorphism $\Gamma_{C} \cong \mathbb{G}_{\mathrm{m}}$ over $\mathbb{F}_{q}(t)$.

Proof. It is easy enough to check this theorem directly. However, by Lemma $3.5 .3 \mathcal{T}_{C}$ is equivalent to a $\mathbb{Z}$-graded category of vector spaces over $\bar{k}(t)$ with a fiber functor to $\operatorname{Vec}\left(\mathbb{F}_{q}(t)\right)$, and so its Galois group is $\mathbb{G}_{\mathrm{m}}$ over $\mathbb{F}_{q}(t)$ [11, Ex. II.2.30].

\section{Galois theory of Systems of $\sigma$-Semilinear equations}

In this section we demonstrate how to calculate the Galois group of a $t$-motive as the Galois group of a system of difference equations with respect to the automorphism $\sigma: \bar{k}(t) \rightarrow \bar{k}(t)$. These systems of equations and their Galois groups are similar to systems of linear differential equations and their Galois groups, and one should compare our constructions with [25, Ch. 1], 26, Chs. 1-2], which we have used as guides, as well as [4], 6], 10], 18, 20], 24]. For an example of a Galois group of this type in the context of $t$-motives, see also the proof of [7, Prop. 7.1].

Van der Put and Singer 25] have developed the theory of Picard-Vessiot rings for linear difference equations which is quite useful in our context. However, their treatment generally assumes that the field of constants is algebraically closed. In our case the field of constants is $\mathbb{F}_{q}(t)$, which presents several difficulties. On the other hand, the Picard-Vessiot rings treated in 25 are not always domains, whereas our central Picard-Vessiot rings are domains by construction, which provides several benefits for the characterization of their Galois groups. It is worth noting that some of what is covered here is covered by the theory of Y. André 4, but we present everything from scratch for completeness. We thank the referee for making several useful suggestions for improving the clarity of this section.

\subsection{Solutions of $\sigma$-semilinear equations.}

4.1.1. Fields of definition. Let $F \subseteq K \subseteq L$ be fields together with an automorphism $\sigma: L \rightarrow L$. We say that the triple $(F, K, L)$ is $\sigma$-admissible if

- $\sigma$ restricts to automorphisms of $F$ and $K$;

- $F=F^{\sigma}=K^{\sigma}=L^{\sigma}$;

- $L$ is a separable extension of $K$.

The primary example of $\sigma$-admissible fields that we have in mind is

$$
(F, K, L)=\left(\mathbb{F}_{q}(t), \bar{k}(t), \mathbb{L}\right),
$$

with automorphism $\sigma$ defined as in $\$ 2.2 .5$ by $\sigma(f)=f^{(-1)}$. This example will be important for applications to $t$-motives in $\$ 4.5$. To see that this triple is $\sigma$-admissible, we know that $\mathbb{F}_{q}(t)=\mathbb{F}_{q}(t)^{\sigma}=\bar{k}(t)^{\sigma}$ by definition and that $\mathbb{L}^{\sigma}=\mathbb{F}_{q}(t)$ by Lemma3.3.2 Also, since $\mathbb{L}$ is linearly disjoint from $\bar{k}\left(t^{1 / p}\right)$, it is therefore separable over $\bar{k}(t) 22$, Thm. 26.3]. Henceforth we shall assume that a $\sigma$-admissible triple $(F, K, L)$ has been chosen.

4.1.2. Convention. If $\rho: S \rightarrow R$ is a homomorphism of modules or rings, and $B \in$ $\operatorname{Mat}_{r \times s}(S)$, we let $\rho(B) \in \operatorname{Mat}_{r \times s}(R)$ be the matrix obtained by applying $\rho$ to the entries of $B$.

4.1.3. Definition. Given a matrix $\Phi \in \operatorname{GL}_{r}(K)$, we consider vectors $\psi \in \operatorname{Mat}_{r \times 1}(L)$ that satisfy

$$
\sigma(\psi)=\Phi \psi
$$

In this way, we define a system of $\sigma$-semilinear equations, and $\psi$ is a solution. The set of solutions

$$
\operatorname{Sol}(\Phi):=\left\{\psi \in \operatorname{Mat}_{r \times 1}(L) \mid \sigma(\psi)=\Phi \psi\right\}
$$


is an $F$-vector space.

Lemma 4.1.4. Let $\Phi \in \mathrm{GL}_{r}(K)$. Suppose that $\psi_{1}, \ldots, \psi_{m} \in \operatorname{Sol}(\Phi)$ are linearly independent over $F$. Then they are linearly independent over $L$.

Proof. The proof is in the same spirit as the one for Lemma 3.3.7 and we omit it.

Corollary 4.1.5. Let $\Phi \in \operatorname{Mat}_{r}(K)$. Then $\operatorname{Sol}(\Phi)$ is an F-vector space of dimension at most $r$.

4.1.6. Fundamental matrix of solutions. Given $\Phi \in \mathrm{GL}_{r}(K)$, suppose $\Psi \in \mathrm{GL}_{r}(L)$ satisfies

$$
\sigma(\Psi)=\Phi \Psi .
$$

Then by Lemma 4.1.4 and Corollary 4.1.5, the columns of $\Psi$ form an $F$-basis for $\operatorname{Sol}(\Phi)$. The matrix $\Psi$ is called a fundamental matrix for $\Phi$. It is useful to note that $\Psi^{\prime} \in \mathrm{GL}_{r}(L)$ is another fundamental matrix for $\Phi$ if and only if $\Psi^{-1} \Psi^{\prime}$ is fixed by $\sigma$. That is, if and only if $\Psi^{\prime}=\Psi \delta$ for some $\delta \in \mathrm{GL}_{r}(F)$.

4.2. The difference Galois group. Throughout this section we fix $\Phi \in \mathrm{GL}_{r}(K)$ and suppose that $\Psi \in \mathrm{GL}_{r}(L)$ is a fundamental matrix for $\Phi$ with respect to our $\sigma$-admissible triple $(F, K, L)$.

For a ring $R$, we let $\mathrm{GL}_{r / R}$ denote the $R$-group scheme of $r \times r$ invertible matrices. Its coordinate ring is $R[X, 1 / \operatorname{det} X]$, where $X=\left(X_{i j}\right)$ is an $r \times r$ matrix of independent variables. If $S$ is an $R$-algebra, we will as usual let $G L_{r}(S)$ denote the group of $S$ rational points on $\mathrm{GL}_{r / R}$. For any $R$-scheme $Z$, we let $Z_{S}:=S \times_{R} Z$ be its base extension to an $S$-scheme.

4.2.1. Construction of $\Gamma$. We define a $K$-algebra map $\nu: K[X, 1 / \operatorname{det} X] \rightarrow L$ by setting $\nu\left(X_{i j}\right):=\Psi_{i j}$. We let

$$
\mathfrak{p}:=\operatorname{ker} \nu, \quad \Sigma:=\operatorname{im} \nu=K[\Psi, 1 / \operatorname{det} \Psi] \subseteq L .
$$

We let $\Lambda$ be the fraction field of $\Sigma$. Finally, we let $Z=\operatorname{Spec} \Sigma$. In this way $Z$ is the small closed subscheme of $\mathrm{GL}_{r / K}$ such that $\Psi \in Z(L)$.

Now set $\Psi_{1}, \Psi_{2} \in \mathrm{GL}_{r}\left(L \otimes_{K} L\right)$ to be the matrices such that $\left(\Psi_{1}\right)_{i j}=\Psi_{i j} \otimes 1$ and $\left(\Psi_{2}\right)_{i j}=1 \otimes \Psi_{i j}$, and let $\widetilde{\Psi}:=\Psi_{1}^{-1} \Psi_{2} \in \mathrm{GL}_{r}\left(L \otimes_{K} L\right)$. We define an $F$-algebra map $\mu: F[X, 1 / \operatorname{det} X] \rightarrow L \otimes_{K} L$ by $\mu\left(X_{i j}\right)=\widetilde{\Psi}_{i j}$. We let

$$
\mathfrak{q}:=\operatorname{ker} \mu, \quad \Delta:=\operatorname{im} \mu,
$$

and finally we set $\Gamma=\operatorname{Spec} \Delta$. In this way $\Gamma$ is the smallest closed subscheme of $\mathrm{GL}_{r / F}$ such that $\widetilde{\Psi} \in \Gamma\left(L \otimes_{K} L\right)$. If we wish denote the dependence on $\Psi$, we will write $Z_{\Psi}$ and $\Gamma_{\Psi}$ for these spaces.

Among other things, we will see in Theorem 4.2.11 that $\Gamma$ is a closed subgroup of $\mathrm{GL}_{r / F}$ and that $Z$ is a $\Gamma_{K}$-torsor under right-multiplication.

4.2.2. The automorphisms $\boldsymbol{\sigma}_{0}$ and $\boldsymbol{\sigma}_{1}$. We define a natural $\sigma$-linear automorphisms

$$
\boldsymbol{\sigma}_{0}, \boldsymbol{\sigma}_{1}: L[X, 1 / \operatorname{det} X] \rightarrow L[X, 1 / \operatorname{det} X],
$$

by setting $\boldsymbol{\sigma}_{0} X:=X$ and $\boldsymbol{\sigma}_{1} X:=\Phi X$. We note that $\boldsymbol{\sigma}_{0}$ restricts to an automorphism of $R[X, 1 / \operatorname{det} X]$ for any $F$-subalgebra $R$ of $L$, and that $\boldsymbol{\sigma}_{1}$ induces automorphisms of $K[X, 1 / \operatorname{det} X]$ and $\Sigma[X, 1 / \operatorname{det} X]$. We see that

$$
\sigma_{0} \mathfrak{q}=\mathfrak{q}, \quad \sigma_{1} \mathfrak{p}=\mathfrak{p} .
$$


The first equality is clear since $\mathfrak{q} \subseteq F[X, 1 / \operatorname{det} X]$. For the second, we note that for $h(X) \in K[X, 1 / \operatorname{det} X]$, we have $\boldsymbol{\sigma}_{1}(h)(X)=\boldsymbol{\sigma}_{0}(h)(\Phi X)$, and so

$$
\boldsymbol{\sigma}_{1}(h)(\Psi)=\boldsymbol{\sigma}_{0}(h)(\Phi \Psi)=\boldsymbol{\sigma}_{0}(h)(\sigma(\Psi))=\sigma(h(\Psi)) .
$$

Thus, $\boldsymbol{\sigma}_{1} \mathfrak{p}=\mathfrak{p}$. This equality implies further that $\nu \boldsymbol{\sigma}_{1}=\sigma \nu$. The following lemma provides a correspondence between the contraction and extension of ideals in $L[X, 1 / \operatorname{det} X]$. See also [26, Lem. 1.23].

Lemma 4.2.3. The functions between sets of ideals,

$$
\begin{array}{ccc}
\{\mathfrak{a} \subseteq F[X, 1 / \operatorname{det} X]\} & \longleftrightarrow & \left\{\mathfrak{b} \subseteq L[X, 1 / \operatorname{det} X] \mid \boldsymbol{\sigma}_{0} \mathfrak{b}=\mathfrak{b}\right\}, \\
\mathfrak{a} & \rightarrow & (\mathfrak{a}) \\
\mathfrak{b} \cap F[X, 1 / \operatorname{det} X] & \leftarrow & \mathfrak{b}
\end{array}
$$

are bijections.

Proof. Since $\sigma_{0}$ is trivial on $F[X, 1 / \operatorname{det} X] \subseteq L[X, 1 / \operatorname{det} X]$, these maps are welldefined. One knows already that $(\mathfrak{a}) \cap F[X, 1 / \operatorname{det} X]=\mathfrak{a}$ for all ideals $\mathfrak{a} \subseteq F[X, 1 / \operatorname{det} X]$ (see [34, §VII.11]). Now let $\mathfrak{b} \subseteq L[X, 1 / \operatorname{det} X]$ be an ideal with $\boldsymbol{\sigma}_{0} \mathfrak{b}=\mathfrak{b}$, and let $\mathfrak{a}:=\mathfrak{b} \cap F[X, 1 / \operatorname{det} X]$. Letting $\left\{g_{i}\right\}_{i \in I}$ be an $F$-basis of $F[X, 1 / \operatorname{det} X]$, we have that $\left\{g_{i}\right\}_{i \in I}$ is an $L$-basis of $L[X, 1 / \operatorname{det} X]$. For $h \in \mathfrak{b}$ we write $h=\sum b_{i} g_{i}, b_{i} \in L$, and we let $l(h)$ be the number of $i \in I$ for which $b_{i} \neq 0$. We show that $h \in(\mathfrak{a})$ by induction on $l(h)$. If $l(h)=0$ the result is clear. If $l(h)=1$, then $h=b g$ for some $b \in L^{\times}$and $g \in\left\{g_{i}\right\}$. Moreover, then $g \in \mathfrak{a}$. Now suppose that $l(h)>1$. By multiplying by an element of $L$ we can assume that $b_{i_{1}}=1$ and that $b_{i_{2}} \in L \backslash F$ for some $i_{1}, i_{2} \in I$. (If all $b_{i} \in F$, then $h \in \mathfrak{a}$.) One sees that

$$
l\left(\boldsymbol{\sigma}_{0} h-h\right)<l(h),
$$

and since $\boldsymbol{\sigma}_{0} \mathfrak{b}=\mathfrak{b}$, we have $\boldsymbol{\sigma}_{0} h-h \in \mathfrak{b}$. Therefore, $\boldsymbol{\sigma}_{0}(h)-h \in(\mathfrak{a})$. Similarly, $\boldsymbol{\sigma}_{0}\left(b_{i_{2}}^{-1} h\right)-b_{i_{2}}^{-1} h \in(\mathfrak{a})$. However,

$$
\left(\sigma\left(b_{i_{2}}^{-1}\right)-b_{i_{2}}^{-1}\right) h=\left(\boldsymbol{\sigma}_{0}\left(b_{i_{2}}^{-1} h\right)-b_{i_{2}}^{-1} h\right)-\sigma\left(b_{i_{2}}^{-1}\right) \cdot\left(\boldsymbol{\sigma}_{0} h-h\right) .
$$

The left-hand side is non-zero, and the right-hand side is in $(\mathfrak{a})$. Therefore $h \in(\mathfrak{a})$.

Proposition 4.2.4. Define a morphism of affine L-schemes $\phi:=Z_{L} \rightarrow \mathrm{GL}_{r / L}$ so that on points $u \mapsto \Psi^{-1} u$ for $u \in Z(\bar{L})$. Then $\phi$ factors through an isomorphism $\phi^{\prime}: Z_{L} \rightarrow \Gamma_{L}$ of affine L-schemes.

Proof. For commutative rings $R \subseteq S$ and for any ideal $I$ in $R[X, 1 / \operatorname{det} X]$, we let $I_{S}$ denote its extension to $S[X, 1 / \operatorname{det} X]$. Now the ideal $\mathfrak{p} \subseteq K[X, 1 / \operatorname{det} X]$ is the defining ideal of the $K$-scheme $Z$, and $\mathfrak{q} \subseteq F[X, 1 / \operatorname{det} X]$ is the defining ideal of of the $F$-scheme $\Gamma$. If we set

$$
\alpha: L[X, 1 / \operatorname{det} X] \rightarrow L[X, 1 / \operatorname{det} X]
$$

to be the $L$-algebra homomorphism determined by setting $\alpha(X)=\Psi^{-1} X$, then the map

$$
\bar{\alpha}: L[X, 1 / \operatorname{det} X] \rightarrow L[X, 1 / \operatorname{det} X] / \mathfrak{p}_{L},
$$

induced by $\alpha$, is the map $\phi$ on the level of coordinate rings. It then suffices to prove that $\mathfrak{q}_{L}=\alpha^{-1} \mathfrak{p}_{L}$.

As noted in 4.2.2, we have that $\boldsymbol{\sigma}_{0} \mathfrak{q}_{L}=\mathfrak{q}_{L}$ and $\boldsymbol{\sigma}_{1} \mathfrak{p}_{L}=\mathfrak{p}_{L}$. Furthermore,

$$
\boldsymbol{\sigma}_{1} \alpha X=\boldsymbol{\sigma}_{1}\left(\Psi^{-1} X\right)=(\sigma \Psi)^{-1}\left(\boldsymbol{\sigma}_{1} X\right)=\Psi^{-1} X=\alpha \boldsymbol{\sigma}_{0} X,
$$

and so $\boldsymbol{\sigma}_{1} \alpha=\alpha \boldsymbol{\sigma}_{0}$, which implies that

$$
\boldsymbol{\sigma}_{0} \alpha^{-1} \mathfrak{p}_{L}=\alpha^{-1} \mathfrak{p}_{L}
$$


By Lemma 4.2.3, it follows that $\alpha^{-1} \mathfrak{p}_{L}$ is generated by $\alpha^{-1} \mathfrak{p}_{L} \cap F[X, 1 / \operatorname{det} X]$.

Now we regard $L \otimes_{K} L$ as an $L$-algebra through the map $f \mapsto f \otimes 1$. If we let $\tilde{\mu}$ : $L[X, 1 / \operatorname{det} X] \rightarrow L \otimes_{K} L$ be the unique $L$-algebra homomorphism such that $\tilde{\mu} X=\Psi_{2}$, then we note that the composition

$$
F[X, 1 / \operatorname{det} X] \stackrel{\alpha}{\rightarrow} L[X, 1 / \operatorname{det} X] \stackrel{\tilde{\mu}}{\rightarrow} L \otimes_{K} L
$$

is in fact $\mu$. Since $L$ is a field, the map $L[X, 1 / \operatorname{det} X] / \mathfrak{p}_{L} \rightarrow L \otimes_{K} L$ induced by $\tilde{\mu}$ is injective. Therefore,

$$
\mathfrak{q}=\alpha^{-1} \mathfrak{p}_{L} \cap F[X, 1 / \operatorname{det} X],
$$

and by our argument in the previous paragraph, $\mathfrak{q}_{L}=\alpha^{-1} \mathfrak{p}_{L}$.

Corollary 4.2.5. The ideal $\mathfrak{p} \subseteq K[X, 1 / \operatorname{det} X]$ is maximal among proper $\boldsymbol{\sigma}_{1}$-invariant ideals.

Proof. Let $\mathfrak{m} \supseteq \mathfrak{p}$ be a proper ideal of $K[X, 1 / \operatorname{det} X]$ such that $\boldsymbol{\sigma}_{1} \mathfrak{m} \subseteq \mathfrak{m}$. Because $K[X, 1 / \operatorname{det} X]$ is noetherian, it follows that $\boldsymbol{\sigma}_{1} \mathfrak{m}=\mathfrak{m}$. Now $\alpha^{-1} \mathfrak{m}_{L} \supseteq \alpha^{-1} \mathfrak{p}_{L}=\mathfrak{q}_{L}$, and we see easily that $\sigma_{0} \alpha^{-1} \mathfrak{m}_{L}=\alpha^{-1} \mathfrak{m}_{L}$. Therefore, by Lemma 4.2.3,

$$
\alpha^{-1} \mathfrak{m}_{L}=\left(\alpha^{-1} \mathfrak{m}_{L} \cap F[X, 1 / \operatorname{det} X]\right)_{L} .
$$

Let $\mathfrak{a} \subseteq F[X, 1 / \operatorname{det} X]$ be a maximal ideal that contains $\alpha^{-1} \mathfrak{m}_{L} \cap F[X, 1 / \operatorname{det} X]$, and let $E:=F[X, 1 / \operatorname{det} X] / \mathfrak{a}$, which is a finite extension of $F$. By Lemma 4.2 .3 we see that $\mathfrak{a}=\mathfrak{a}_{L} \cap F[X, 1 / \operatorname{det} X]$, and it follows that there is an isomorphism $\beta$ : $L[X, 1 / \operatorname{det} X] / \mathfrak{a}_{L} \stackrel{\sim}{\rightarrow} L \otimes_{F} E$. Now if we consider the maps

$$
\Pi: L[X, 1 / \operatorname{det} X] \stackrel{\alpha^{-1}}{\rightarrow} L[X, 1 / \operatorname{det} X] \stackrel{\beta}{\rightarrow} L \otimes_{F} E,
$$

we see that $\mathfrak{m}_{L} \subseteq \operatorname{ker} \Pi$. If we let $\pi: K[X, 1 / \operatorname{det} X] \rightarrow L \otimes_{F} E$ be the restriction of $\Pi$, then easily $\mathfrak{m} \subseteq \operatorname{ker} \pi$ and $\operatorname{ker} \pi$ is a proper ideal. Moreover, since $\alpha^{-1} \boldsymbol{\sigma}_{1}=\boldsymbol{\sigma}_{0} \alpha^{-1}$, it follows that $\operatorname{ker} \pi$ is a $\sigma_{1}$-invariant ideal of $K[X, 1 / \operatorname{det} X]$. Therefore, the maximality of $\mathfrak{m}$ implies that $\mathfrak{m}=\operatorname{ker} \pi$.

Now let $\Psi^{\prime} \in \mathrm{GL}_{r}\left(L \otimes_{F} E\right)$ be defined by $\Psi_{i j}^{\prime}=\pi\left(X_{i j}\right)$. The automorphism $\sigma$ on $L$ extends to an automorphism of $L \otimes_{F} E$ by acting by the identity on $E$, and it is easily seen that $\left(L \otimes_{F} E\right)^{\sigma}=E$. In this way $\sigma\left(\Psi^{\prime}\right)=\Phi \Psi^{\prime}$, and this implies that the matrix $\delta:=\left(\Psi^{\prime}\right)^{-1} \Psi \in \mathrm{GL}_{r}(E)$. Now $\delta$ induces an automorphism on $\left(K \otimes_{F} E\right)[X, 1 / \operatorname{det} X]$ via

$$
\delta \cdot h(X):=h(X \delta)
$$

If we extend $\pi$ to $\pi^{\prime}:\left(K \otimes_{F} E\right)[X, 1 / \operatorname{det} X] \rightarrow L \otimes_{F} E$ by the identity on $E$, then we see that we have the extended ideals

$$
\mathfrak{p}_{K \otimes_{F} E}=\mathfrak{p} \otimes_{F} E \subseteq \mathfrak{m} \otimes_{F} E \subseteq \operatorname{ker} \pi^{\prime}=\delta \cdot\left(\mathfrak{p} \otimes_{F} E\right) .
$$

But $\left(K \otimes_{F} E\right)[X, 1 / \operatorname{det} X]$ is a noetherian ring, and so $\mathfrak{p} \otimes_{F} E \subseteq \delta \cdot\left(\mathfrak{p} \otimes_{F} E\right)$ implies that $\mathfrak{p} \otimes_{F} E=\delta \cdot\left(\mathfrak{p} \otimes_{F} E\right)$. Thus,

$$
\mathfrak{p} \otimes_{F} E=\mathfrak{m} \otimes_{F} E=\delta \cdot\left(\mathfrak{p} \otimes_{F} E\right) .
$$

Now $\left(K \otimes_{F} E\right)[X, 1 / \operatorname{det} X]$ is a free $K[X, 1 / \operatorname{det} X]$-module, since $E$ is a vector space over $F$, and is therefore faithfully flat over $K[X, 1 / \operatorname{det} X]$. So by intersecting with $K[X, 1 / \operatorname{det} X]$ we see that $\mathfrak{p}=\mathfrak{m}$ (see [22, Thm. 7.5$]$ ). 
4.2.6. Contracted ideals of $\Sigma[X, 1 / \operatorname{det} X]$. The following lemma is a companion to Lemma 4.2.3, and relies on the preceding corollary. See also [25, Lem. 1.11].

Lemma 4.2.7. Let $\mathfrak{b} \subset \Sigma[X, 1 / \operatorname{det} X]$ be an ideal that is $\boldsymbol{\sigma}_{0}$-invariant. Then $\mathfrak{b}$ is generated by $\mathfrak{b} \cap F[X, 1 / \operatorname{det} X]$.

Proof. Let $\mathfrak{a}:=\mathfrak{b} \cap F[X, 1 / \operatorname{det} X]$. Let $\left\{g_{i}\right\}_{i \in I}$ be an $F$-basis for $F[X, 1 / \operatorname{det} X]$ such that $I=I_{\mathfrak{a}} \cup I_{1}$, where $\left\{g_{i}\right\}_{i \in I_{\mathfrak{a}}}$ is an $F$-basis for $\mathfrak{a}$. Choose a subset $J \subseteq I_{1}$ minimal so that $\mathfrak{b} \cap \sum_{i \in J} \Sigma g_{i}$ contains a non-zero element of $\mathfrak{b} /(\mathfrak{a})$. Pick $j \in J$, and let

$$
\mathfrak{m}:=\left\{b \in \Sigma \mid \exists \sum_{i \in J} b_{i} g_{i} \in \mathfrak{b} /(\mathfrak{a}), b_{j}=b\right\}
$$

Since $\boldsymbol{\sigma}_{0} \mathfrak{b}=\mathfrak{b}$, and since each $g_{i}$ is fixed by $\boldsymbol{\sigma}_{0}$, it follows that $\mathfrak{m}$ is a non-zero $\sigma$ invariant ideal of $\Sigma$. However, by Corollary 4.2.5, $\Sigma$ has no $\sigma$-invariant ideals other than $\{0\}$ and $\Sigma$. Thus, $\mathfrak{m}=\Sigma$. Therefore there exists $h \in \mathfrak{b}$ so that $h=\sum_{i \in J} b_{i} g_{i}$ $\bmod (\mathfrak{a})$ and $b_{j}=1$. Now $\boldsymbol{\sigma}_{0}(h)-h$ is supported on a proper subset of $J$ modulo (a), and so it must be 0 modulo (a) by the minimality of $J$. Therefore each $b_{i} \in F$, and thus $\sum_{i \in J} b_{i} g_{i} \in(\mathfrak{a})$, which is a contradiction.

Proposition 4.2.8. Define a morphism of affine $K$-schemes $\psi: Z \times Z \rightarrow Z \times \mathrm{GL}_{r / K}$ so that on points $(u, v) \mapsto\left(u, u^{-1} v\right)$ for $u, v \in Z(\bar{K})$. Then $\psi$ factors through an isomorphism $Z \times Z \rightarrow Z \times \Gamma_{K}$ of affine $K$-schemes.

Proof. Again we work on the level of coordinate rings and maintain conventions and definitions in the proof of Proposition 4.2.4. The ring $\Sigma \subseteq L$ is isomorphic to the coordinate ring of $Z$ over $K$. Likewise, the ring $\Sigma[X, 1 / \operatorname{det} X] / \mathfrak{p}_{\Sigma}$ is the coordinate ring of $Z \times Z$, and $\Sigma[X, 1 / \operatorname{det} X] / \mathfrak{q}_{\Sigma}$ is the coordinate ring of $Z \times \Gamma_{K}$. The $L$-algebra automorphism $\alpha$ in the proof of the previous proposition restricts to an automorphism of $\Sigma[X, 1 / \operatorname{det} X]$, and in this way the homomorphism

$$
\bar{\alpha}: \Sigma[X, 1 / \operatorname{det} X] \rightarrow \Sigma[X, 1 / \operatorname{det} X] / \mathfrak{p}_{\Sigma}
$$

induced by $\alpha$ represents the morphism $\psi$ of affine $K$-schemes. We then need to show that $\mathfrak{q}_{\Sigma}=\alpha^{-1} \mathfrak{p}_{\Sigma}$.

Let $\mathfrak{a}=\alpha^{-1} \mathfrak{p}_{\Sigma} \cap F[X, 1 / \operatorname{det} X]$. By Lemma 4.2.7

$$
\alpha^{-1} \mathfrak{p}_{\Sigma}=\mathfrak{a}_{\Sigma}
$$

Then as in the proof of Proposition 4.2.4. $\mathfrak{q}_{L}=\alpha^{-1} \mathfrak{p}_{L}$ and both are now equal to $\mathfrak{a}_{L}$. Since $\mathfrak{q}$ and $\mathfrak{a}$ are both ideals in $F[X, 1 / \operatorname{det} X]$ and $F$ and $L$ are fields, it follows that $\mathfrak{q}=\mathfrak{a}$.

Lemma 4.2.9. Let $G$ be a group, and let $A$ and $B$ be subsets of $G, A$ non-empty, such that the map

$$
(u, v) \mapsto\left(u, u^{-1} v\right): A \times A \rightarrow A \times G
$$

factors through a bijection $\phi: A \times A \rightarrow A \times B$. Then $B$ is a subgroup of $G$ and $A$ is stable under right-multiplication by elements of $B$. Moreover, under the action of $B$ by right-multiplication, A becomes a principal homogeneous space for $B$.

Proof. This is a simple exercise.

4.2.10. The Galois group $\Gamma$. The previous propositions and lemmas culminate in the following theorem, saying that $\Gamma$ is in fact an affine group scheme. We call the group $\Gamma$, or $\Gamma_{\Psi}$ if we wish to recall the dependence on $\Psi$, the Galois group of the system $\sigma(\Psi)=\Phi \Psi$. 
Theorem 4.2.11. Let $(F, K, L)$ be a $\sigma$-admissible triple for an automorphism $\sigma: L \rightarrow$ L. Suppose we have $\Phi \in G L_{r}(K)$ and $\Psi \in G L_{r}(L)$ so that $\sigma(\Psi)=\Phi \Psi$. Then $\Gamma:=\Gamma_{\Psi}$ is a closed F-subgroup scheme of $\mathrm{GL}_{r / F}$, and the closed $K$-subscheme $Z:=Z_{\Psi}$ of $\mathrm{GL}_{r / K}$ is stable under right-multiplication by $\Gamma_{K}$ and is a $\Gamma_{K}$-torsor.

Proof. Since $Z(\Sigma)$ is non-empty, Propositions 4.2 .4 and 4.2 .8 imply that $(u, v) \mapsto$ $\left(u, u^{-1} v\right): Z(\Sigma) \times Z(\Sigma) \rightarrow Z(\Sigma) \times \Gamma(\Sigma)$ is a bijection. Lemma 4.2 .9 and the Yoneda lemma [32, $\S 1.2-1.4]$ imply that $\Gamma_{\Sigma}$ is a subgroup of $\mathrm{GL}_{r / \Sigma}$ and that $Z_{\Sigma}$ is a $\Gamma_{\Sigma}$-torsor. Since the inclusion $F \rightarrow \Sigma$ is faithfully flat, we see that $\Gamma$ is a closed $F$-subgroup scheme of $\mathrm{GL}_{r / F}$ by flat descent [32, §17.1-17.3]. Similarly, since the inclusion $K \rightarrow \Sigma$ is faithfully flat, $Z$ admits the structure of a $\Gamma_{K}$-torsor.

4.3. Criterion for smoothness. We continue with the notation of the previous section, and in particular have fixed a $\sigma$-admissible triple $(F, K, L)$ together with $\Phi \in$ $\mathrm{GL}_{r}(K), \Psi \in \mathrm{GL}_{r}(L)$ satisfying $\sigma(\Psi)=\Phi \Psi$. In this section, we explore when $\Gamma$ is smooth over $\bar{F}$, that is, when the coordinate ring of $\Gamma_{\bar{F}}$ is reduced.

Theorem 4.3.1. Suppose $K$ is algebraically closed in the fraction field $\Lambda$ of $\Sigma$. Then

(a) The $K$-scheme $Z$ is absolutely irreducible and is smooth over $\bar{K}$.

(b) The F-scheme $\Gamma$ is absolutely irreducible and is smooth over $\bar{F}$.

(c) The dimension of $\Gamma$ over $F$ is equal to the transcendence degree of $\Lambda$ over $K$.

Proof. The ideal $\mathfrak{p} \subseteq K[X, 1 / \operatorname{det} X]$ is prime. The field $\Lambda$ is separable over $K$, since it is a subfield of $L$. That $K$ is algebraically closed in $\Lambda$ then implies that $\mathfrak{p}_{\bar{K}} \subseteq$ $\bar{K}[X, 1 / \operatorname{det} X]$ is prime [34, VII.11, Thm. 39]. Thus $Z$ is smooth over $\bar{K}$. Because $\Gamma_{\bar{K}} \cong Z_{\bar{K}}, \Gamma$ must be smooth over $\bar{F}$. By construction the transcendence degree of $\Lambda$ over $K$ is equal to the dimension of $Z$, which is equal to the dimension of $\Gamma$.

4.3.2. The case $\left(\mathbb{F}_{q}(t), \bar{k}(t), \mathbb{L}\right)$. This case is of particular interest to our applications to $t$-motives. It turns out that in this case, all Galois groups are smooth, via the following proposition. We continue with our usual notation.

Proposition 4.3.3. Suppose $(F, K, L)=\left(\mathbb{F}_{q}(t), \bar{k}(t), \mathbb{L}\right)$, and suppose that $\Phi \in \mathrm{GL}_{r}(\bar{k}(t))$ and $\Psi \in \mathrm{GL}_{r}(\mathbb{L})$ satisfy $\Psi^{(-1)}=\Phi \Psi$. Then $\bar{k}(t)$ is algebraically closed in $\Lambda=\bar{k}(t)(\Psi)$.

Proof. Let $f \in \Lambda \cap \overline{k(t)}$, and consider the field $H:=\bar{k}\left(t ; f^{(i)}: i \in \mathbb{Z}\right)$ obtained by adjoining all of the twists of $f$ to $\bar{k}(t)$. Each $f^{(i)}$ is algebraic over $\bar{k}(t)$, and so $H / \bar{k}(t)$ is algebraic. Since $\Lambda$ is finitely generated as a field over $\bar{k}(t)$, so is $H$. Thus $[H: \bar{k}(t)]<\infty$. Furthermore, $H$ is invariant under $\sigma$ and $\sigma^{-1}$.

The field $H$ is the function field of a smooth projective curve $X$ over $\bar{k}$, and the inclusion $\bar{k}(t) \subseteq H$ provides a surjective morphism $X \rightarrow \mathbb{P} \frac{1}{k}$ over $\bar{k}$. Now $\sigma: H \rightarrow H$ induces an automorphism $\tau: X \rightarrow X$ as a scheme over $\mathbb{F}_{q}$. Because $\sigma$ leaves the integral closure of $\bar{k}[t]$ in $H$ invariant, the points $\infty_{1}, \ldots, \infty_{d}$ in $X$ above the point $\infty$ in $\mathbb{P} \frac{1}{k}$ are permuted by $\sigma$. Thus we can construct an effective divisor $I$ of $X$ such that $\tau(I)=I$ and $\operatorname{Supp}(I)=\left\{\infty_{1}, \ldots, \infty_{d}\right\}$. Now for $N \geq 1$ sufficiently large, the field $H$ is generated over $\bar{k}(t)$ by the functions in the finite dimensional $\bar{k}$-vector space

$$
S:=\Gamma(X, N \cdot I) \subseteq H .
$$

By our assumptions on $I$, this space is invariant under $\sigma$ and $\sigma^{-1}$. If the entries of $\mathbf{f}:=\left[f_{1}, \ldots, f_{m}\right]^{\text {tr }}$ form a $\bar{k}$-basis for $S$, then there is a matrix $A \in \mathrm{GL}_{m}(\bar{k})$ so that $\sigma(\mathbf{f})=A \mathbf{f}$. If $\mathbf{g} \in \operatorname{Mat}_{m \times 1}(S)$ and $\mathbf{g}=B \mathbf{f}$ for some $B \in \mathrm{GL}_{m}(\bar{k})$, then

$$
\sigma(\mathbf{g})=B^{(-1)} A B^{-1} \mathbf{g} \text {. }
$$


By the theory of Lang isogenies [19], we can pick a $B \in \mathrm{GL}_{m}(\bar{k})$ so that

$$
B^{-1} B^{(1)}=A^{(1)},
$$

and if we let $\mathbf{g}:=B \mathbf{f}$, then $\sigma(\mathbf{g})=\mathbf{g}$. Thus $S$ contains a $\bar{k}$-basis $\mathbf{g}$ that is fixed by $\sigma$, and $H=\bar{k}(t, \mathbf{g})$. Let $g$ be an entry of $\mathbf{g}$. Then $g \in \overline{k(t)} \cap \mathbb{L}^{\sigma}=\mathbb{F}_{q}(t)$. Thus $[H: \bar{k}(t)]=1$.

Corollary 4.3.4. Let $(F, K, L)=\left(\mathbb{F}_{q}(t), \bar{k}(t), \mathbb{L}\right)$, and suppose that $\Phi \in \mathrm{GL}_{r}(\bar{k}(t))$ and $\Psi \in \mathrm{GL}_{r}(\mathbb{L})$ satisfy $\sigma(\Psi)=\Phi \Psi$. Then the Galois group $\Gamma$ of $\Psi$ is smooth over $\overline{\mathbb{F}_{q}(t)}$.

4.4. The Galois action. In this section we will assume that $K$ is algebraically closed in $\Lambda$, and so in particular by Theorem 4.3.1, $\Gamma_{\bar{F}}$ and $Z_{\bar{K}}$ are reduced and irreducible.

4.4.1. $\sigma$-automorphisms of $\Sigma$ and $\Lambda$. Let $\operatorname{Aut}_{\sigma}(\Sigma / K)$ denote the group of automorphisms of $\Sigma$ over $K$ that commute with $\sigma$. Similarly we define $\operatorname{Aut}_{\sigma}(\Lambda / K)$. In fact, it is true that

$$
\operatorname{Aut}_{\sigma}(\Sigma / K)=\operatorname{Aut}_{\sigma}(\Lambda / K)
$$

Indeed every automorphism $\xi \in \operatorname{Aut}_{\sigma}(\Sigma / K)$ extends uniquely to an automorphism in $\operatorname{Aut}_{\sigma}(\Lambda / K)$. On the other hand, if $\eta \in \operatorname{Aut}_{\sigma}(\Lambda / K)$, then as matrices in $\operatorname{Mat}_{r}(L)$, $\sigma(\eta(\Psi))=\eta(\sigma(\Psi))=\eta(\Phi \Psi)=\Phi \eta(\Psi)$. Thus, $\eta(\Psi)=\Psi \gamma$ for some $\gamma \in \mathrm{GL}_{r}(F)$, and so $\eta(\Psi) \in \operatorname{Mat}_{r}(\Sigma)$. Therefore $\eta$ restricted to $\Sigma$ takes values in $\Sigma$.

4.4.2. The action of $\Gamma(F)$. For $\gamma \in \Gamma(F)$, we have an automorphism of $K$-schemes $\gamma: Z \rightarrow Z$ defined by right multiplication by $\gamma$. On the level of coordinate rings, the induced map is

$$
\gamma=(h(X) \mapsto h(X \gamma)): \Sigma \rightarrow \Sigma,
$$

which is a $K$-linear automorphism that commutes with the action of $\sigma$. Thus we have a group homomorphism,

$$
\kappa: \Gamma(F) \rightarrow \operatorname{Aut}_{\sigma}(\Lambda / K)
$$

which is easily seen to be injective. Now if $\delta \in \operatorname{Aut}_{\sigma}(\Lambda / K)$, then $\delta$ induces an automorphism of the non-empty $Z(\Lambda)$ that is right-multiplication by an element of $\gamma \in \Gamma(\Lambda)$. That $\delta$ commutes with $\sigma$ implies that $\gamma \in \Gamma\left(\Lambda^{\sigma}\right)=\Gamma(F)$. Thus $\kappa$ is an isomorphism.

4.4.3. Base extensions. Given our $\sigma$-admissible triple $(F, K, L)$, we choose an extension of $\sigma$ to an automorphism of $\bar{L}$. Then $\bar{L}^{\sigma}$ is an algebraic extension of $F$. Indeed, the monic irreducible polynomial of any $h \in \bar{L}^{\sigma}$ over $L$ must have coefficients in $L^{\sigma}=F$. Thus if we let $\mathbf{F}=\bar{L}^{\sigma}$, then $(\mathbf{F}, \bar{K}, \bar{L})$ is a $\sigma$-admissible triple. The Galois group $\Gamma^{\prime}$ defined by the system $\sigma(\Psi)=\Phi \Psi$ defined with respect to $(\mathbf{F}, \bar{K}, \bar{L})$ is seen to be $\Gamma_{\mathbf{F}}$ by Propositions 4.2.4 and 4.2.8. If we let $\widetilde{\Sigma}$ be the coordinate of $Z_{\bar{K}}$ and $\widetilde{\Lambda}$ be its fraction field, then we see that

$$
\Gamma(\mathbf{F}) \cong \operatorname{Aut}_{\sigma}(\widetilde{\Sigma} / \bar{K})=\operatorname{Aut}_{\sigma}(\widetilde{\Lambda} / \bar{K}) .
$$

Furthermore, for $n \geq 1$, let $\mathbf{F}_{n}=\bar{L}^{\sigma^{n}}$, and suppose that $\left(\mathbf{F}_{n}, \bar{K}, \bar{L}\right)$ is $\sigma^{n}$-admissible. Then $\Psi$ is a fundamental matrix for $\Phi_{n}:=\sigma^{n-1}(\Phi) \cdots \sigma(\Phi) \Phi$. Again by Propositions 4.2.4 and 4.2.8, we see that the Galois group of this system of equations is $\Gamma_{\mathbf{F}_{n}}$. And thus,

$$
\Gamma\left(\mathbf{F}_{n}\right) \cong \operatorname{Aut}_{\sigma^{n}}(\widetilde{\Sigma} / \bar{K})=\operatorname{Aut}_{\sigma^{n}}(\widetilde{\Lambda} / \bar{K}) .
$$

4.4.4. Galois action for $\Gamma(\bar{F})$. Continuing with the notation of the previous paragraphs, suppose that $\bar{F}=\cup \mathbf{F}_{n}$. Then every element of $\Gamma(\bar{F})$ induces an automorphism of $\widetilde{\Lambda} / \bar{K}$ that commutes with $\sigma^{n}$ for all $n \gg 0$. In this case, we will call this the induced action of $\Gamma(\bar{F})$ on $\widetilde{\Lambda}$. 
4.4.5. The case $\left(\mathbb{F}_{q}(t), \bar{k}(t), \mathbb{L}\right)$. It is worth pointing out that the situation is quite nice in our usual setting. For $n \geq 1$, the triple $\left(\mathbb{F}_{q^{n}}(t), \bar{k}(t), \mathbb{L}\right)$ is $\sigma^{n}$-admissible. As in $\$ 2.2 .5$, there is a canonical extension of $\sigma$ to $\mathbb{K}\langle\langle t\rangle \supseteq \supseteq \mathbb{L}$. Furthermore, we see that

$$
\overline{\mathbb{L}}^{\sigma^{n}}=\overline{k(t)}^{\sigma^{n}}={\overline{\mathbb{F}_{q}(t)}}^{\sigma^{n}}=: \mathbf{F}_{n},
$$

and so $\left(\mathbf{F}_{n}, \overline{k(t)}, \overline{\mathbb{L}}\right)$ is a $\sigma^{n}$-admissible triple. Every element of $\overline{\mathbb{F}_{q}(t)}$ is fixed by some power of $\sigma$, and so

$$
\overline{\mathbb{F}_{q}(t)}=\bigcup_{n \geq 1} \mathbf{F}_{n} .
$$

We now return to the general situation, but it is important to note that the following theorem applies to Galois groups in the usual $\left(\mathbb{F}_{q}(t), \bar{k}(t), \mathbb{L}\right)$ setting.

Theorem 4.4.6. Let $\Phi \in \mathrm{GL}_{r}(K)$, and suppose that $\Psi \in \mathrm{GL}_{r}(L)$ is a fundamental matrix for $\Phi$. Assume that $K$ is algebraically closed in $\Lambda=K(\Psi)$. Fix an extension of $\sigma$ to $\bar{L}$, and let $\mathbf{F}_{n}:=\bar{L}^{\sigma^{n}}$. Suppose that $\left(\mathbf{F}_{n}, \bar{K}, \bar{L}\right)$ is $\sigma^{n}$-admissible for each $n \geq 1$, and suppose that $\bar{F}=\cup \mathbf{F}_{n}$. Let $\widetilde{\Sigma}$ be the coordinate ring of $Z_{\bar{K}}$ and let $\widetilde{\Lambda}$ be its fraction field, both considered subrings of $\bar{L}$.

(a) The subfield of $\widetilde{\Lambda}$ fixed by $\Gamma(\bar{F})$ is $\bar{K}$.

(b) The elements of $\Lambda$ fixed by $\Gamma(\bar{F})$ are precisely $K$.

Proof. See [25, Lem. 1.28]. Suppose $f \in \widetilde{\Lambda}$ is fixed by $\Gamma(\bar{F})$. We consider $f \in \widetilde{\Lambda}$ to be a function $f: Z_{\bar{K}} \rightarrow \mathbb{P}_{\bar{K}}^{1}$. For $i=1,2$, we consider the two maps of $\bar{K}$-schemes

$$
g_{i}: Z_{\bar{K}} \times \Gamma_{\bar{K}} \rightarrow Z_{\bar{K}} \times Z_{\bar{K}} \stackrel{\pi_{i}}{\rightarrow} Z_{\bar{K}} \stackrel{f}{\rightarrow} \mathbb{P} \frac{1}{K},
$$

where $\pi_{i}$ is the $i$-th projection. Because $f$ is $\Gamma(\bar{F})$-invariant and because $\Gamma(\bar{F})$ is dense in $\Gamma_{\bar{K}}$ since $\Gamma$ is smooth over $\bar{F}$, we must have $g_{1}=g_{2}$. Therefore, $f \circ \pi_{1}=f \circ \pi_{2}$, which implies that $f$ is constant. This proves part (a). Part (b) follows from part (a) and the assumption that $K$ is algebraically closed in $\Lambda$.

4.4.7. Remark. If $\Gamma(F)$ is Zariski dense in $\Gamma$, then it follows that $\Lambda^{\Gamma(F)}=\bar{k}(t)$.

4.5. The group $\Gamma$ and $t$-motives. Given a $t$-motive $M$, we defined the Galois group $\Gamma_{M}$ of $M$ in 33.5.2. Associated to $M$ we can also choose a matrix $\Phi \in \mathrm{GL}_{r}(\bar{k}(t))$ that represents multiplication by $\boldsymbol{\sigma}$ on $M$. Let $\Psi \in \mathrm{GL}_{r}(\mathbb{L})$ be a rigid analytic trivialization of $\Phi$. We will show that $\Gamma_{M}$ is isomorphic to $\Gamma:=\Gamma_{\Psi}$ over $\mathbb{F}_{q}(t)$.

4.5.1. $t$-motives and $\sigma$-semilinear equations. Let $M$ be a $t$-motive. We fix the following notation throughout this section. Let $\mathbf{m}$ be a basis for $M$, and let $\Phi \in \mathrm{GL}_{r}(\bar{k}(t))$ represent multiplication by $\boldsymbol{\sigma}$ on $M$. We pick a rigid analytic trivialization $\Psi \in \mathrm{GL}_{r}(\mathbb{L})$ for $M$, which is at the same time a fundamental matrix for $\Phi$.

Let $M_{v}^{u}:=M^{\otimes u} \otimes\left(M^{\vee}\right)^{\otimes v}$. Because $\mathcal{T}_{M}$ is Tannakian, if $N$ is any $t$-motive in $\mathcal{T}_{M}$, then $N$ is the subquotient of a direct sum of various $M_{v}^{u}$, and vice versa. It follows from Propositions 3.3.9(b) and 3.3.11(b) that the entries of a fundamental matrix $\Psi_{N}$ for $N$ are in $\Sigma$, and in fact we can take $\Psi_{N} \in \mathrm{GL}_{s}(\Sigma)$ for some $s$.

For an $\mathbb{F}_{q}(t)$-algebra $R$, we let $\Sigma^{(R)}:=R \otimes_{\mathbb{F}_{q}(t)} \Sigma$.

Lemma 4.5.2. For any t-motive $N$ in $\mathcal{T}_{M}$ and $\mathbb{F}_{q}(t)$-algebra $R$, the natural map,

$$
\Sigma^{(R)} \otimes_{\mathbb{F}_{q}(t)} N^{\mathrm{B}} \rightarrow \Sigma^{(R)} \otimes_{\bar{k}(t)} N
$$

is bijective. 
Proof. Let $\kappa$ be the map defined in the statement of the lemma. Thus as above we can pick a basis $\mathbf{n}$ for $N$ and a rigid analytic trivialization $\Psi_{N} \in \mathrm{GL}_{s}(\Sigma)$ with respect to $\mathbf{n}$. By Proposition 3.3.9(b), $\Psi_{N}^{-1} \mathbf{n}$ is an $\mathbb{F}_{q}(t)$-basis for $N^{\mathrm{B}}$. Now $1 \otimes\left(\Psi_{N}^{-1} \mathbf{n}\right)$ is a $\Sigma^{(R)}$-basis of $\Sigma^{(R)} \otimes_{\mathbb{F}_{q}(t)} N^{\mathrm{B}}$ (here and elsewhere $1 \otimes A$ for a matrix $A$ is the matrix of the same dimension whose entries are each tensored by 1 on the left). If $\mathbf{f} \in \operatorname{Mat}_{1 \times s}\left(\Sigma^{(R)}\right)$, then

$$
\kappa\left((\mathbf{f} \otimes 1) \cdot\left(1 \otimes\left(\Psi_{N}^{-1} \mathbf{n}\right)\right)\right)=\left(\mathbf{f} \Psi_{N}^{-1} \otimes 1\right) \cdot(1 \otimes \mathbf{n}) .
$$

The entries of $\left(\Psi_{N}^{-1} \otimes 1\right) \cdot(1 \otimes \mathbf{n})$ are in the image of $\kappa$, and

$$
\Psi_{N} \cdot\left(\Psi_{N}^{-1} \otimes 1\right) \cdot(1 \otimes \mathbf{n})=1 \otimes \mathbf{n} .
$$

Thus $\kappa$ is surjective. Since $\Psi_{N} \in \mathrm{GL}_{s}(\Sigma)$, the map $\kappa$ is bijective.

Theorem 4.5.3. Let $M$ be a t-motive, and let $N$ be a t-motive in $\mathcal{T}_{M}$. If we consider $N^{\mathrm{B}}$ to be an algebraic group over $\mathbb{F}_{q}(t)$, then there is a natural representation

$$
\xi_{N}: \Gamma \rightarrow \operatorname{GL}\left(N^{\mathrm{B}}\right)
$$

over $\mathbb{F}_{q}(t)$ that is functorial in $N$.

Proof. Since every $t$-motive $N$ in $\mathcal{T}_{M}$ is constructed from $M$ via tensor products, duals, and subquotients, to define this representation it suffices to define it on $M^{\mathrm{B}}$ itself. Functoriality in $N$ will be automatic.

To define the representation on $M^{\mathrm{B}}$, it suffices by the Yoneda lemma [32, $\left.\$ 1.2-1.4\right]$ to define a representation

$$
\xi_{M}^{(R)}: \Gamma(R) \rightarrow \mathrm{GL}\left(R \otimes_{\mathbb{F}_{q}(t)} M^{\mathrm{B}}\right)
$$

for every $\mathbb{F}_{q}(t)$-algebra $R$ and show that it is functorial in $R$. Let $R$ be an $\mathbb{F}_{q}(t)$-algebra, and let $\gamma \in \Gamma(R)$. Define

$$
\Xi^{(R)}(\gamma):=\gamma \otimes 1=(h(\Psi) \otimes m \mapsto h(\Psi \gamma) \otimes m): \Sigma^{(R)} \otimes_{\bar{k}(t)} M \rightarrow \Sigma^{(R)} \otimes_{\bar{k}(t)} M,
$$

which is an isomorphism of $\bar{k}(t)$-vector spaces. Now by Lemma 4.5.2, $R \otimes_{\mathbb{F}_{q}(t)} M^{\mathrm{B}}$ spans $\Sigma^{(R)} \otimes_{\bar{k}(t)} M$ as a $\Sigma^{(R)}$-module. Let $\xi^{(R)}(\gamma)$ be the restriction of $\Xi^{(R)}(\gamma)$ to $R \otimes_{\mathbb{F}_{q}(t)} M^{\mathrm{B}}$.

We claim that the image of $\xi^{(R)}(\gamma)$ is $R \otimes_{\mathbb{F}_{q}(t)} M^{\mathrm{B}}$. Indeed, since $\Psi^{-1} \mathbf{m}$ forms an $\mathbb{F}_{q}(t)$-basis of $M^{\mathrm{B}}$ by Proposition 3.3.9(b), for $\mathbf{f} \in \operatorname{Mat}_{1 \times r}(R)$, we have

$$
\xi^{(R)}(\gamma): \mathbf{f} \cdot\left(1 \otimes \Psi^{-1}\right) \mathbf{m} \mapsto \mathbf{f} \cdot \gamma^{-1}\left(1 \otimes \Psi^{-1}\right) \mathbf{m} .
$$

Thus $\xi^{(R)}(\gamma)$ is an $R$-linear automorphism of $R \otimes_{\mathbb{F}_{q}(t)} M^{\mathrm{B}}$. It is straightforward to check that this is construction is functorial in $R$, and so we have defined a homomorphism $\xi_{M}: \Gamma \rightarrow \mathrm{GL}\left(M^{\mathrm{B}}\right)$

Corollary 4.5.4. Let $M$ be a $t$-motive. The representation $\xi_{M}: \Gamma \rightarrow \operatorname{GL}\left(M^{\mathrm{B}}\right)$ is faithful.

Proof. As defined in the the proof of the previous theorem we see easily that $\xi^{(R)}$ : $\Gamma(R) \rightarrow \mathrm{GL}\left(R \otimes_{\mathbb{F}_{q}(t)} M^{\mathrm{B}}\right)$ is injective for all $\mathbb{F}_{q}(t)$-algebras $R$.

4.5.5. The functor $\xi_{M}$. For a $t$-motive $M$, if $\Phi \in \mathrm{GL}_{r}(\bar{k}(t))$ represents multiplication by $\sigma$ on $M$ and if $\Psi \in \mathrm{GL}_{r}(\mathbb{L})$ is a rigid analytic trivialization of $\Phi$, then Theorem 4.5.3 defines a functor

$$
\xi_{M}: \mathcal{T}_{M} \rightarrow \boldsymbol{\operatorname { R e p }}\left(\Gamma, \mathbb{F}_{q}(t)\right) .
$$

It is straightforward to check that $\xi_{M}$ is a tensor functor. Let

$$
\eta_{M}: \operatorname{Rep}\left(\Gamma_{M}, \mathbb{F}_{q}(t)\right) \stackrel{\sim}{\rightarrow} \mathcal{T}_{M}
$$


be the equivalence of categories defined in $\$ 3.5 .2$. Letting

$$
F: \operatorname{Rep}\left(\Gamma, \mathbb{F}_{q}(t)\right) \rightarrow \operatorname{Vec}\left(\mathbb{F}_{q}(t)\right)
$$

be the forgetful functor, we see immediately that $\omega_{M}=F \circ \xi_{M}$. Thus by [11, Cor. II.2.9], there is a unique homomorphism $\pi_{M}: \Gamma \rightarrow \Gamma_{M}$ over $\mathbb{F}_{q}(t)$ so that the natural functor $\tau_{M}: \operatorname{Rep}\left(\Gamma_{M}, \mathbb{F}_{q}(t)\right) \rightarrow \boldsymbol{R e p}\left(\Gamma, \mathbb{F}_{q}(t)\right)$ induced by $\pi_{M}$ satisfies

$$
\xi_{M} \circ \eta_{M}=\tau_{M} .
$$

Proposition 4.5.6. Let $M$ be a t-motive. Suppose that $\Phi \in \mathrm{GL}_{r}(\bar{k}(t))$ represents multiplication by $\boldsymbol{\sigma}$ on $M$ and that $\Psi \in \mathrm{GL}_{r}(\mathbb{L})$ is a rigid analytic trivialization for $\Phi$. Then the functor

$$
\xi_{M}: \mathcal{T}_{M} \rightarrow \operatorname{Rep}\left(\Gamma, \mathbb{F}_{q}(t)\right)
$$

is fully faithful.

Proof. For any $t$-motives $N$ and $P$ in $\mathcal{T}_{M}$, there is a natural isomorphism of $\mathbb{F}_{q}(t)$ vector spaces, $\operatorname{Hom}_{\mathcal{T}_{M}}(P, N) \cong \operatorname{Hom}_{\mathcal{T}_{M}}(\mathbf{1}, \operatorname{Hom}(P, N))$. Thus it suffices to prove full faithfulness when $P=\mathbf{1}$. Now $\operatorname{Hom}_{\mathcal{T}_{M}}(\mathbf{1}, N) \cong N \cap N^{\mathrm{B}}=\{n \in N \mid \boldsymbol{\sigma} n=n\}$, and this provides an injection $\operatorname{Hom}_{\mathcal{T}_{M}}(\mathbf{1}, N) \hookrightarrow \operatorname{Hom}_{\Gamma}\left(\mathbf{1}^{\mathrm{B}}, N^{\mathrm{B}}\right)$.

Conversely suppose that $\phi: \mathbf{1}^{\mathrm{B}} \rightarrow N^{\mathrm{B}}$ is a $\Gamma$-morphism. Pick a $\bar{k}(t)$-basis $\mathbf{n}$ for $N$. Then $\phi(1)=\mathbf{h}(\Psi) \cdot \mathbf{n}$ for some $\mathbf{h}(\Psi) \in \operatorname{Mat}_{1 \times s}(\Sigma)$ by Lemma 4.5.2, Let $E / \mathbb{F}_{q}(t)$ be a finite extension of fields. We see that for $\gamma \in \Gamma(E)$, the action of $\xi^{(E)}(\gamma):=$ $\xi_{M}^{(E)}(N)(\gamma)$ on $E \otimes_{\mathbb{F}_{q}(t)} N^{\mathrm{B}}$ is simply the restriction of the natural map $\Xi^{(E)}(\gamma)=$ $1 \otimes \gamma: \Sigma^{(E)} \otimes_{\bar{k}(t)} N \rightarrow \Sigma^{(E)} \otimes_{\bar{k}(t)} N$ to $E \otimes_{\mathbb{F}_{q}(t)} N^{\mathrm{B}}$. Since $\phi$ is a $\Gamma$-morphism, it follows that $\xi^{(E)}(\gamma)(\phi(1))=\phi(1)$ for all $\gamma \in \Gamma(E)$. Thus,

$$
\mathbf{h}(\Psi) \cdot \mathbf{n}=\phi(1)=\xi^{(E)}(\gamma)(\phi(1))=\mathbf{h}(\Psi \gamma) \cdot \mathbf{n}, \quad \gamma \in \Gamma(E) .
$$

Because $\mathbf{n}$ is a $\Sigma^{(E)}$-basis of $\Sigma^{(E)} \otimes_{\bar{k}(t)} N$, the entries of $\mathbf{h}(\Psi)$ must each be fixed by every $\gamma \in \Gamma(E)$. By varying over all $E / \mathbb{F}_{q}(t)$ finite, Theorem 4.4 .6 implies that $\mathbf{h}(\Psi) \in \operatorname{Mat}_{1 \times s}(\bar{k}(t))$. Thus $\phi(1) \in N \cap N^{\mathrm{B}}$.

Lemma 4.5.7. Let $\Phi \in \mathrm{GL}_{r}(\bar{k}(t))$, and suppose that $\Psi \in \mathrm{GL}_{r}(\mathbb{L})$ is a fundamental matrix for $\Phi$. Suppose that $W \subseteq \Lambda^{\oplus s}$ is a vector subspace over $\Lambda$ such that for every finite extension of fields $E / \mathbb{F}_{q}(t)$,

$$
\Gamma(E) \cdot\left(E \otimes_{\mathbb{F}_{q}(t)} W\right) \subseteq E \otimes_{\mathbb{F}_{q}(t)} W .
$$

Then $W$ has a system of defining equations over $\bar{k}(t)$.

Proof. Suppose that $W$ has dimension $s-m$, and let $A(\Psi) \in \operatorname{Mat}_{m \times s}(\Lambda)$ be a coefficient matrix for a system of defining equations for $W$. By changing the order of the variables if necessary, we can use Gaussian elimination on $A(\Psi)$ to obtain

$$
G(\Psi)=\left[I_{m}, C(\Psi)\right]
$$

where $C(\Psi) \in \operatorname{Mat}_{m \times(s-m)}(\Lambda)$. Both $A(\Psi)$ and $G(\Psi)$ provide coefficient matrices for equations for $W$, and so it suffices to show that $C(\Psi)$ has entries in $\bar{k}(t)$.

Let $E / \mathbb{F}_{q}(t)$ be a finite extension of fields. Since $E \otimes_{\mathbb{F}_{q}(t)} W$ is invariant under $\Gamma(E)$, it follows that, for every $\gamma \in \Gamma(E)$, the matrix $G\left(\Psi \gamma^{-1}\right)$ is also the coefficient matrix of a defining set of equations for $E \otimes_{\mathbb{F}_{q}(t)} W$. Now the columns of the matrix $\left[-C(\Psi), I_{s-m}\right]^{\text {tr }} \in \operatorname{Mat}_{m \times s}(\Lambda)$ form a basis for $W$. Thus,

$$
\left[\begin{array}{ll}
I_{m} & C\left(\Psi \gamma^{-1}\right)
\end{array}\right] \cdot\left[\begin{array}{c}
-C(\Psi) \\
I_{s-m}
\end{array}\right]=0, \quad \forall \gamma \in \Gamma(E),
$$


and so $C(\Psi \gamma)=C(\Psi), \quad \forall \gamma \in \Gamma(E)$. After varying over all $E / \mathbb{F}_{q}(t)$ finite, it follows from Theorem 4.4.6 that $C(\Psi) \in \operatorname{Mat}_{m \times(s-m)}(\bar{k}(t))$.

Proposition 4.5.8. Let $M$ be a t-motive. Suppose that $\Phi \in \mathrm{GL}_{r}(\bar{k}(t))$ represents multiplication by $\boldsymbol{\sigma}$ on $M$ and that $\Psi \in \mathrm{GL}_{r}(\mathbb{L})$ is a rigid analytic trivialization for $\Phi$. For every $t$-motive $N$ in $\mathcal{T}_{M}$ and every $\Gamma$-subrepresentation $V$ of $N^{\mathrm{B}}$, there is a sub-t-motive $P \subseteq N$ so that $\xi_{M}(P)=V$.

Proof. Pick a $\bar{k}(t)$-basis $\mathbf{n} \in$ Mat $_{s \times 1}$ for $N$ with $\boldsymbol{\sigma} \mathbf{n}=\Phi_{N} \mathbf{n}$, and let $\Psi_{N} \in \mathrm{GL}_{s}(\mathbb{L})$ be a rigid analytic trivialization for $\Phi_{N}$. Let $\mathbf{v} \in \operatorname{Mat}_{v \times 1}\left(N^{\mathrm{B}}\right)$ be an $\mathbb{F}_{q}(t)$-basis for $V$, and extend $\mathbf{v}$ to a basis $\mathbf{u}$ of $N^{\mathrm{B}}, \mathbf{u}=[\mathbf{v}, \mathbf{w}]^{\text {tr }}$. By Lemma 4.5.2, there is a $H(\Psi) \in \mathrm{GL}_{s}(\Sigma)$ so that $\mathbf{u}=H(\Psi) \cdot \mathbf{n}$. We note that $H(\Psi)=\delta^{-1} \Psi_{N}^{-1}$ for some $\delta \in \mathrm{GL}_{s}\left(\mathbb{F}_{q}(t)\right)$ by Proposition 3.3.9(b).

Let $E / \mathbb{F}_{q}(t)$ be a finite extension of fields, and let $\gamma \in \Gamma(E)$. The action of $\gamma$ on $E \otimes_{\mathbb{F}_{q}(t)} N^{\mathrm{B}}$ is given by the restriction of $\Xi^{(E)}$ as in the proof of Proposition 4.5.6 to $E \otimes_{\mathbb{F}_{q}(t)} N^{\mathrm{B}}$. Thus,

$$
\xi^{(E)}(\gamma)(\mathbf{u})=H(\Psi \gamma) \mathbf{n}=H(\Psi \gamma) H(\Psi)^{-1} \mathbf{u} .
$$

Since $V$ is invariant under $\Gamma$, it follows that the upper right $v \times(s-v)$ block of $H(\Psi \gamma) H(\Psi)^{-1}$ is 0 for every $\gamma \in \Gamma(E)$. Let $D(\Psi) \in \operatorname{Mat}_{s \times(s-v)}(\Lambda)$ be the $s-v$ right-most columns of $H(\Psi)^{-1}$, and consider the subspace $W \subseteq \operatorname{Mat}_{1 \times s}(\Lambda)$,

$$
W=\left\{\mathbf{x} \in \operatorname{Mat}_{1 \times s}(\Lambda) \mid \mathbf{x} \cdot D(\Psi)=0\right\} .
$$

By our considerations on $H(\Psi)$ at the end of the preceding paragraph, we see from Lemma 4.5.7 that $W$ has a set of defining equations over $\bar{k}(t)$. Thus there is a $C \in$ $\operatorname{Mat}_{v \times s}(\bar{k}(t))$ of maximal rank so that $C \cdot D(\Psi)=0$. Extend $C$ to a matrix $B \in$ $\mathrm{GL}_{s}(\bar{k}(t))$ such that $C$ forms the top rows of $B$. Now let $\mathbf{n}^{\prime}=B \cdot \mathbf{n}=[\mathbf{p}, \mathbf{q}]^{\text {tr }}$, with $\boldsymbol{\sigma} \mathbf{n}^{\prime}=\Phi^{\prime} \mathbf{n}^{\prime}$, and let $P$ be the $\bar{k}(t)$-span of $\mathbf{p}=C \cdot \mathbf{n}$. Then

$$
\boldsymbol{\sigma}\left[\begin{array}{l}
\mathbf{p} \\
\mathbf{q}
\end{array}\right]=\boldsymbol{\sigma}(B \cdot \mathbf{n})=\boldsymbol{\sigma}\left(B H(\Psi)^{-1} H(\Psi) \mathbf{n}\right)=\left(B \cdot H(\Psi)^{-1}\right)^{(-1)} H(\Psi) \cdot B^{-1} \cdot\left[\begin{array}{l}
\mathbf{p} \\
\mathbf{q}
\end{array}\right] .
$$

By construction, the upper right-hand $v \times(s-v)$ block of $B \cdot H(\Psi)^{-1}$ is 0 . Thus,

$$
\boldsymbol{\sigma}\left[\begin{array}{l}
\mathbf{p} \\
\mathbf{q}
\end{array}\right]=\left[\begin{array}{cc}
\Phi_{P} & 0 \\
* & *
\end{array}\right] \cdot\left[\begin{array}{l}
\mathbf{p} \\
\mathbf{q}
\end{array}\right]=\Phi^{\prime} \cdot\left[\begin{array}{l}
\mathbf{p} \\
\mathbf{q}
\end{array}\right] .
$$

Since $\Phi^{\prime} \in \mathrm{GL}_{s}(\bar{k}(t))$, it follows that $\Phi_{P} \in \mathrm{GL}_{v}(\bar{k}(t))$. Thus $P$ is a sub-t-motive of $N$. Furthermore, as $H(\Psi)^{-1}=\Psi_{N} \delta, \delta \in \mathrm{GL}_{s}\left(\mathbb{F}_{q}(t)\right)$, it follows that $B \cdot H(\Psi)^{-1}$ is a rigid analytic trivialization of $\Phi^{\prime}$. If we set take $\Psi_{P}$ to be the upper left-hand block of $B \cdot H(\Psi)^{-1}$, then $\Psi_{P}$ is a rigid analytic trivialization for $\Phi_{P}$. Moreover, it follows that $P^{\mathrm{B}}=V$ by Proposition 3.3 .9 (b).

Proposition 4.5.9. Let $M$ be a t-motive. Suppose that $\Phi \in \mathrm{GL}_{r}(\bar{k}(t))$ represents multiplication by $\sigma$ on $M$ and that $\Psi \in \mathrm{GL}_{r}(\mathbb{L})$ is a rigid analytic trivialization for $\Phi$. To every representation $W$ in $\operatorname{Rep}\left(\Gamma, \mathbb{F}_{q}(t)\right)$ there is a t-motive $N$ in $\mathcal{T}_{M}$ so that $W$ is isomorphic to a subquotient of $\xi_{M}(N)$.

Proof. The representation $M^{\mathrm{B}}$ is faithful by Corollary 4.5.4. Thus any object in $\operatorname{Rep}\left(\Gamma, \mathbb{F}_{q}(t)\right)$ is isomorphic to a subquotient of a direct sum of representations of the form $\left(M^{\mathrm{B}}\right)_{v}^{u}:=\left(M^{\mathrm{B}}\right)^{\otimes u} \otimes\left(\left(M^{\mathrm{B}}\right)^{\vee}\right)^{\otimes v}$. Since $\xi_{M}\left(M_{v}^{u}\right)=\left(M_{v}^{u}\right)^{\mathrm{B}} \cong\left(M^{\mathrm{B}}\right)_{v}^{u}$, the proposition follows. 
Theorem 4.5.10. Let $M$ be a t-motive. Suppose that $\Phi \in \mathrm{GL}_{r}(\bar{k}(t))$ represents multiplication by $\boldsymbol{\sigma}$ on $M$ and that $\Psi \in \mathrm{GL}_{r}(\mathbb{L})$ is a rigid analytic trivialization for $\Phi$. Then the functor

$$
\xi_{M}: \mathcal{T}_{M} \rightarrow \operatorname{Rep}\left(\Gamma, \mathbb{F}_{q}(t)\right)
$$

is an equivalence of Tannakian categories. Equivalently, the homomorphism $\pi_{M}: \Gamma \rightarrow$ $\Gamma_{M}$ is an isomorphism over $\mathbb{F}_{q}(t)$.

Proof. By Propositions 4.5.6 and 4.5.8 the map $\pi_{M}$ is faithfully flat [11, Prop. II.2.21(a)]. By Proposition 4.5.9, $\pi_{M}$ is a closed immersion [11, Prop. II.2.21(b)]. Thus $\pi_{M}$ is an isomorphism of affine group schemes over $\mathbb{F}_{q}(t)$.

4.5.11. Remark. Although we have focused on objects in the category $\mathcal{T}$, the above theorem is true (with the same proof) if $M$ is replaced by simply a rigid analytically trivial pre- $t$-motive.

\section{Galois Groups And transcendence}

In this section we first recall the linear independence criterion introduced in [2] by Anderson, Brownawell, and the author, and one of its applications to $t$-motives. We then link this together with our study of the Galois groups of certain $t$-motives, whose matrices representing multiplication by $\boldsymbol{\sigma}$ have entries in $\bar{k}[t]$ and whose fundamental matrices have entries in $\mathbb{E}$. These $t$-motives include as a subset rigid analytically trivial Anderson $t$-motives. In what follows our primary goal will be to consider the fundamental matrix $\Psi$ associated to such a $t$-motive $M$ and to equate the transcendence degree over $\bar{k}$ of $\Psi(\theta)$ and the dimension of the Galois group of $M$.

\subsection{Linear independence criterion.}

Theorem 5.1.1 ([2, Thm. 3.1.1]). Let $\Phi \in \operatorname{Mat}_{r}(\bar{k}[t])$ be given such that $\operatorname{det} \Phi=$ $c(t-\theta)^{s}, c \in \bar{k}^{\times}$, and suppose that $\psi \in \operatorname{Mat}_{r \times 1}(\mathbb{E})$ satisfies

$$
\psi^{(-1)}=\Phi \psi \text {. }
$$

For every $\rho \in \operatorname{Mat}_{1 \times r}(\bar{k})$ such that $\rho \psi(\theta)=0$, there is a $P \in \operatorname{Mat}_{1 \times r}(\bar{k}[t])$ so that $P(\theta)=\rho$ and $P \psi=0$.

5.1.2. Connection with solutions of $\sigma$-semilinear equations. At first glance at the above theorem, the solutions $\psi$ of the $\sigma$-semilinear equation associated to $\Phi$ are quite special in that their entries are assumed to be in $\mathbb{E}$. However, the following proposition demonstrates that this situation is not unusual.

Proposition 5.1.3 ([2, Prop. 3.1.3]). Suppose we are given $\Phi \in \operatorname{Mat}_{r}(\bar{k}[t])$ and $\psi \in$ $\operatorname{Mat}_{r \times 1}(\mathbb{T})$ so that

$$
\operatorname{det} \Phi(0) \neq 0, \quad \psi^{(-1)}=\Phi \psi .
$$

Then we necessarily have $\psi \in \operatorname{Mat}_{r \times 1}(\mathbb{E})$.

5.1.4. Connection with left $\bar{k}[t ; \boldsymbol{\sigma}]$-modules. The following is a variation on [2, Prop. 4.4.3] with slightly milder hypotheses. We do not assume that the representing matrix $\Phi$ is one directly associated to an Anderson $t$-motive. However, we do obtain the same equality of dimensions (with the same proof).

Proposition 5.1.5 ([2, Prop. 4.4.3]). Let $\Phi \in \operatorname{Mat}_{r}(\bar{k}[t])$ and $\psi \in \operatorname{Mat}_{r \times 1}(\mathbb{E})$ be given as in Theorem 5.1.1. Let $N$ be the $\bar{k}[t]$-span in $\mathbb{E}$ of the entries of $\psi$, and let $V$ be the $\bar{k}$-span in $\overline{k_{\infty}}$ of the entries of $\psi(\theta)$. Then $\operatorname{rk}_{\bar{k}[t]} N=\operatorname{dim}_{\bar{k}} V$. 
Proof. Let $N_{1}:=\left\{P \in \operatorname{Mat}_{1 \times r}(\bar{k}[t]) \mid P \psi=0\right\}$. We then obtain an exact sequence of $\bar{k}[t]$-modules,

$$
0 \rightarrow N_{1} \rightarrow \operatorname{Mat}_{1 \times r}(\bar{k}[t]) \rightarrow N \rightarrow 0,
$$

where the second map is given by $P \mapsto P \psi$. It is easy to check that this is an exact sequence of left $\bar{k}[t ; \boldsymbol{\sigma}]$-modules. Every $\bar{k}[t]$-basis for $N_{1}$ can be extended to a basis of $\operatorname{Mat}_{1 \times r}(\bar{k}[t])$, and so the number of $\bar{k}$-linearly independent relations of $\bar{k}$-linear dependence among the entries of $\psi(\theta)$ is at least as great $\operatorname{as~}_{\bar{k}[t]} N_{1}$. Thus $\operatorname{rk}_{\bar{k}[t]} N \geq \operatorname{dim}_{\bar{k}} V$. Moreover, Theorem 5.1.1 implies that every $\bar{k}$-linear relation among the entries of $\psi(\theta)$ lifts to a $\bar{k}[t]$-linear relation among the entries of $\psi$. Thus $\operatorname{rk}_{\bar{k}[t]} N \leq \operatorname{dim}_{\bar{k}} V$.

\subsection{Dimensions and transcendence degrees.}

5.2.1. Rigid analytic trivializations over $\mathbb{E}$. Let $M$ be a $t$-motive. Suppose that $\Phi \in$ $\mathrm{GL}_{r}(\bar{k}(t)) \cap \operatorname{Mat}_{r}(\bar{k}[t])$ represents multiplication by $\boldsymbol{\sigma}$ on $M$ and that $\operatorname{det} \Phi=c(t-\theta)^{s}$, $c \in \bar{k}^{\times}$. An important observation is that by Propositions 3.3.9(c) and 5.1.3 there is a rigid analytic trivialization $\Psi$ for $\Phi$ such that $\Psi \in \mathrm{GL}_{r}(\mathbb{T}) \cap \operatorname{Mat}_{r}(\mathbb{E})$.

Theorem 5.2.2. Let $M$ be a t-motive, and let $\Gamma_{M}$ be its Galois group. Suppose that $\Phi \in \mathrm{GL}_{r}(\bar{k}(t)) \cap \operatorname{Mat}_{r}(\bar{k}[t])$ represents multiplication by $\boldsymbol{\sigma}$ on $M$ and that $\operatorname{det} \Phi=$ $c(t-\theta)^{s}, c \in \bar{k}^{\times}$. Let $\Psi$ be a rigid analytic trivialization of $\Phi$ in $\mathrm{GL}_{r}(\mathbb{T}) \cap \operatorname{Mat}_{r}(\mathbb{E})$. Finally, let $L$ be the subfield of $\overline{k_{\infty}}$ generated over $\bar{k}$ by the entries of $\Psi(\theta)$. Then

$$
\text { tr. } \operatorname{deg}_{\bar{k}} L=\operatorname{dim} \Gamma_{M} \text {. }
$$

Proof. By Theorem 4.5.10, the groups $\Gamma_{M}$ and $\Gamma_{\Psi}$ are isomorphic. Moreover, by Theorem 4.3.1, their dimension is the same as tr. $\operatorname{deg}_{\bar{k}(t)} \Lambda$, where $\Lambda=\bar{k}(t)(\Psi) \subseteq \mathbb{L}$. Now let $Q=\bar{k}[\Psi(\theta)] \subseteq L$, and let $S=\bar{k}(t)[\Psi] \subseteq \Lambda$. Then as rings,

$$
Q \cong \bar{k}\left[X_{i j}\right] / \mathfrak{a}, \quad S \cong \bar{k}(t)\left[X_{i j}\right] / \mathfrak{b},
$$

for ideals $\mathfrak{a}$ and $\mathfrak{b}$. For $d \geq 1$, let $\bar{k}\left[X_{i j}\right]_{d}$ and $\mathfrak{a}_{d}$ denote the elements of $\bar{k}\left[X_{i j}\right]$ and $\mathfrak{a}$ of total degree $\leq d$, and let $Q_{d} \subseteq Q$ correspond to their quotient. Similarly define $\bar{k}(t)\left[X_{i j}\right]_{d}, \mathfrak{b}_{d}$, and $S_{d}$.

Fix $d \geq 1$. Now for any $n \geq 1$, the entries of $\Psi^{\otimes n}$ comprise all monomials of total degree $n$ in the $\Psi_{i j}$. If $\psi$ is a column of $\Psi^{\otimes n}$, then $\psi^{(-1)}=\Phi^{\otimes n} \psi$. Thus let $\bar{\psi} \in \operatorname{Mat}_{N \times 1}(\mathbb{E})$ be the column vector whose entries are the concatenation of 1 and each of the columns of $\Psi^{\otimes n}$ for $n \leq d$. (Here $N=\left(r^{2 d+2}-1\right) /\left(r^{2}-1\right)$.) Then if $\bar{\Phi} \in \operatorname{Mat}_{N}(\bar{k}[t]) \cap \mathrm{GL}_{N}(\bar{k}(t))$ is the block diagonal matrix

$$
\bar{\Phi}:=[1] \oplus \Phi^{\oplus r} \oplus\left(\Phi^{\otimes 2}\right)^{\oplus r^{2}} \oplus \cdots\left(\Phi^{\otimes d}\right)^{\oplus r^{d}},
$$

it follows that $\bar{\psi}^{(-1)}=\bar{\Phi} \bar{\psi}$. Now it is easy to see that $Q_{d}$ is the $\bar{k}$-span of the columns of $\bar{\psi}(\theta)$ and that $S_{d}$ is the $\bar{k}(t)$-span of the columns of $\bar{\psi}$. Since $\bar{\Phi}$ and $\bar{\psi}$ satisfy the hypotheses for Proposition 5.1.5, we see that for all $d \geq 1$,

$$
\operatorname{dim}_{\bar{k}} Q_{d}=\operatorname{dim}_{\bar{k}(t)} S_{d}
$$

Thus the homogenizations of $Q$ and $S$ have the same Hilbert series (see 34, Ch. VII, $\S 12])$, and so tr. $\operatorname{deg}_{\bar{k}} L=\operatorname{tr} \cdot \operatorname{deg}_{\bar{k}(t)} \Lambda$.

\section{Application to Carlitz logarithms}

\subsection{Carlitz logarithms and $t$-motives.}


6.1.1. The power series $L_{\alpha}$. For $\alpha \in \bar{k}^{\times}$with $|\alpha|_{\infty}<|\theta|_{\infty}^{q /(q-1)}$, define the power series

$$
L_{\alpha}(t):=\alpha+\sum_{i=1}^{\infty} \frac{\alpha^{q^{i}}}{\left(t-\theta^{q}\right)\left(t-\theta^{q^{2}}\right) \cdots\left(t-\theta^{q^{i}}\right)} .
$$

It is easy to show that $L_{\alpha} \in \mathbb{T}$ and that moreover, $L_{\alpha}(z)$ converges for all $z \in \mathbb{K}$ with $|z|_{\infty}<|\theta|_{\infty}^{q}$. By $\$ 1.2 .2$ we see that

$$
L_{\alpha}(\theta)=\log _{C}(\alpha) .
$$

Furthermore, as a power series in $\mathbb{T}, L_{\alpha}$ also satisfies the functional equation

$$
L_{\alpha}^{(-1)}=\alpha^{(-1)}+\frac{L_{\alpha}}{t-\theta} .
$$

6.1.2. $t$-motives for Carlitz logarithms. Fix $\alpha_{1}, \ldots, \alpha_{r} \in \bar{k}^{\times}$with $\left|\alpha_{i}\right|_{\infty}<|\theta|_{\infty}^{q /(q-1)}$ for $i=1, \ldots, r$. Set

$$
\Phi:=\Phi\left(\alpha_{1}, \ldots, \alpha_{r}\right):=\left[\begin{array}{cccc}
t-\theta & 0 & \cdots & 0 \\
\alpha_{1}^{(-1)}(t-\theta) & 1 & \cdots & 0 \\
\vdots & \vdots & \ddots & \vdots \\
\alpha_{r}^{(-1)}(t-\theta) & 0 & \cdots & 1
\end{array}\right] \in \operatorname{Mat}_{r+1}(\bar{k}[t]) .
$$

Note that $\Phi$ defines a pre-t-motive $X:=X\left(\alpha_{1}, \ldots, \alpha_{r}\right)$ that is an extension of $\mathbf{1}^{r}$ by the Carlitz motive $C$ :

$$
0 \rightarrow C \rightarrow X \rightarrow \mathbf{1}^{r} \rightarrow 0 .
$$

In spite of the restrictions on $\alpha_{1}, \ldots, \alpha_{r}$, we will be able to use the objects $X\left(\alpha_{1}, \ldots, \alpha_{r}\right)$ to accommodate all Carlitz logarithms using Lemma 6.4.1.

Proposition 6.1.3. Let $\alpha_{1}, \ldots, \alpha_{r} \in \bar{k}^{\times}$with $\left|\alpha_{i}\right|_{\infty}<|\theta|_{\infty}^{q /(q-1)}$ for $i=1, \ldots, r$. The pre-t-motive $X=X\left(\alpha_{1}, \ldots, \alpha_{r}\right)$ is a t-motive.

Proof. We prove first that $X$ is rigid analytically trivial and then that $X$ is an object in $\mathcal{T}$. Define

$$
\Psi:=\Psi\left(\alpha_{1}, \ldots, \alpha_{r}\right):=\left[\begin{array}{cccc}
\Omega & 0 & \cdots & 0 \\
\Omega L_{\alpha_{1}} & 1 & \cdots & 0 \\
\vdots & \vdots & \ddots & \vdots \\
\Omega L_{\alpha_{r}} & 0 & \cdots & 1
\end{array}\right] \in \mathrm{GL}_{r+1}(\mathbb{T})
$$

It is a simple matter to check that $\Psi$ is a rigid analytic trivialization for $\Phi$ using (6.1.1.1). We note by Proposition 5.1 .3 that the entries of $\Psi$ are in $\mathbb{E}$.

Consider the pre- $t$-motive $C \otimes X$. We claim that $C \otimes X$ is in the essential image of the functor $\mathrm{M} \mapsto M: \mathcal{A R}^{I} \rightarrow \mathcal{R}$ of Theorem 3.4.9, By the definition of the category $\mathcal{T}$ in 3.4 .10 , it will follow that $X$ is a $t$-motive.

Let $\mathrm{M}:=\bar{k}[t]^{r+1}$ with standard $\bar{k}[t]$-basis $m_{0}, \ldots, m_{r}$. Letting $\mathrm{m}:=\left[m_{1}, \ldots, m_{r}\right]^{\operatorname{tr}}$, we give $\mathrm{M}$ the structure of a left $\bar{k}[t ; \boldsymbol{\sigma}]$-module by setting

$$
\boldsymbol{\sigma m}:=(t-\theta) \Phi \mathrm{m} .
$$

Now $\mathrm{M}$ sits in an exact sequence of left $\bar{k}[t ; \boldsymbol{\sigma}]$-modules,

$$
0 \rightarrow \mathrm{C}^{\otimes 2} \rightarrow \mathrm{M} \rightarrow \mathrm{C}^{r} \rightarrow 0,
$$

where $C$ is the Carlitz motive in the category of Anderson $t$-motives of $\$ 3.4 .3$. Since $\mathrm{C}$ and $\mathrm{C}^{\otimes}$ are finitely generated as left $\bar{k}[\sigma]$-modules, so is $\mathrm{M}$, and it follows from [2. Prop. 4.3.2] that $\mathrm{M}$ is free and finitely generated as a left $\bar{k}[\boldsymbol{\sigma}]$-module. Without 
much difficulty, one shows that $\boldsymbol{\sigma} \mathrm{M}=\left\langle(t-\theta)^{2} m_{0},(t-\theta) m_{1}, \ldots,(t-\theta) m_{r}\right\rangle_{\bar{k}[t]}$. Thus $(t-\theta)^{n} \mathrm{M} \subseteq \sigma \mathrm{M}$ for all $n \geq 2$, and $\mathrm{M}$ is an Anderson $t$-motive by 3.4 .1

6.2. The Galois group $\Gamma_{X}$. We continue with the notations of the previous section, including choices of $\alpha_{1}, \ldots, \alpha_{r} \in \bar{k}^{\times}$with $\left|\alpha_{i}\right|_{\infty}<|\theta|_{\infty}^{q /(q-1)}$ for $i=1, \ldots, r$.

6.2.1. The group $G$. Let $G$ be the algebraic subgroup of $\mathrm{GL}_{r+1}$ over $\mathbb{F}_{q}(t)$ such that for all $\mathbb{F}_{q}(t)$-algebras $R$,

$$
G(R)=\left\{\left[\begin{array}{cc}
* & 0 \\
* & I_{r}
\end{array}\right] \in \mathrm{GL}_{r+1}(R)\right\} .
$$

6.2.2. Preliminary calculations. We claim that $\Gamma_{X} \subseteq G$. As in $\$ 4.2$, we can construct the coordinate ring as the image of $\mu: \mathbb{F}_{q}(t)[X, 1 / \operatorname{det} X] \rightarrow \mathbb{L} \otimes_{\bar{k}(t)} \mathbb{L}$, the $\mathbb{F}_{q}(t)$-algebra homomorphism that sends $X$ to $\widetilde{\Psi}=\Psi_{1}^{-1} \Psi_{2}$. As before let $\mathfrak{q}=\operatorname{ker} \mu$. Direct calculation verifies that $X_{i j}-\delta_{i j} \in \mathfrak{q}$ for all $i \geq 1$ and $j \geq 2$, where $\delta_{i j}$ is the usual Kronecker delta. Thus, $\Gamma_{X} \subseteq G$. It will be convenient henceforth to label the non-trivial coordinates of $G \subseteq \mathrm{GL}_{r+1}$ as $X_{0}, \ldots, X_{r}$.

Because the Carlitz motive $C$ is contained in $X$, it is an object in $\mathcal{T}_{X}$, and hence there is a surjection $\pi: \Gamma_{X} \rightarrow \mathbb{G}_{\mathrm{m}}$ over $\mathbb{F}_{q}(t)$ by Theorem 3.5.4 Now under $\nu$ : $\bar{k}(t)\left[X_{0}, X_{0}^{-1}, X_{1}, \ldots, X_{r}\right] \rightarrow \mathbb{L}$, which takes $X$ to $\Psi$, we have $\nu\left(X_{0}\right)=\Omega$. Thus the action of any $\gamma \in \Gamma_{X}\left(\overline{\mathbb{F}_{q}(t)}\right)$ on $\Omega$ agrees with the action of the $X_{0}$-coordinate of $\gamma$ on $\Omega$. That is, the surjection $\pi$ coincides with the natural projection on the $X_{0}$-coordinate of $G$. Let $V$ be the kernel of $\pi$ so that we have an exact sequence of algebraic groups over $\mathbb{F}_{q}(t)$,

$$
1 \rightarrow V \rightarrow \Gamma_{X} \rightarrow \mathbb{G}_{\mathrm{m}} \rightarrow 1 .
$$

The group $V$ is a subgroup of the group of unipotent matrices of $G$, which itself is naturally isomorphic to $\mathbb{G}_{\mathrm{a}}^{r}$. Thus we can think of $V \subseteq \mathbb{G}_{\mathrm{a}}^{r}$ with coordinates $X_{1}, \ldots, X_{r}$.

Proposition 6.2.3. With notation as above, the group $V$ is a linear subspace of $\mathbb{G}_{\mathrm{a}}^{r}$ over $\mathbb{F}_{q}(t)$.

Proof. Since $\Gamma_{X}$ is a smooth over $\mathbb{F}_{q}(t)$ by Theorem 4.3.1, one verifies that the map $\pi$ is surjective on Lie algebras, and hence $V$ is also smooth. Thus it is determined by the Zariski closure of $V\left(\overline{\mathbb{F}_{q}(t)}\right)$ in $\mathbb{G}_{\mathrm{a}}^{r}$. Because $\pi$ is surjective, for any non-zero $\alpha \in \overline{\mathbb{F}_{q}(t)}$, we can choose $\gamma \in \Gamma_{X}\left(\overline{\mathbb{F}_{q}(t)}\right)$ so that $\pi(\gamma)=\alpha$. Suppose that $\mu=\left[\begin{array}{ll}1 & 0 \\ v & I_{r}\end{array}\right] \in V\left(\overline{\mathbb{F}_{q}(t)}\right)$. Then direct calculation gives $\gamma^{-1} \mu \gamma=\left[\begin{array}{cc}1 & 0 \\ \alpha v & I_{r}\end{array}\right] \in V\left(\overline{\mathbb{F}_{q}(t)}\right)$, and thus $V\left(\overline{\mathbb{F}_{q}(t)}\right)$ is a linear subspace of $\mathbb{G}_{a}^{r}\left(\overline{\mathbb{F}_{q}(t)}\right)$. Since $V$ is smooth, its defining equations over $\overline{\mathbb{F}_{q}(t)}$ are linear forms in $X_{1}, \ldots, X_{r}$. These forms can be defined over $\mathbb{F}_{q}(t)$ since $V$ is simply a linear subspace.

6.2.4. Defining polynomials for $\Gamma_{X}$. Because the map $\Gamma_{X} \rightarrow \mathbb{G}_{\mathrm{m}}$ is a smooth morphism over $\mathbb{F}_{q}(t)$, Hilbert's Theorem 90 provides an exact sequence

$$
1 \rightarrow V\left(\mathbb{F}_{q}(t)\right) \rightarrow \Gamma_{X}\left(\mathbb{F}_{q}(t)\right) \rightarrow \mathbb{G}_{\mathrm{m}}\left(\mathbb{F}_{q}(t)\right) \rightarrow 1
$$

by [32, §18.5]. Let $b_{0} \in \mathbb{F}_{q}(t)^{\times} \backslash \mathbb{F}_{q}^{\times}$, and fix a matrix

$$
\gamma=\left[\begin{array}{cccc}
b_{0} & 0 & \cdots & 0 \\
b_{1} & 1 & \cdots & 0 \\
\vdots & \vdots & \ddots & \vdots \\
b_{r} & 0 & \cdots & 1
\end{array}\right] \in \Gamma_{X}\left(\mathbb{F}_{q}(t)\right)
$$

One checks that the Zariski closure in $\Gamma_{X}$ of the cyclic group generated by $\gamma$ is the line in $G$ connecting $\gamma$ to the identity matrix. Translating this line by any element of $V$ 
shows that $\Gamma_{X}$ contains the linear space spanned by $V$ and $\gamma$. Since $\Gamma_{X}$ is irreducible and of dimension 1 greater than the dimension of $V$, we see that $\Gamma_{X}$ is this linear subspace. Moreover, this implies the following proposition.

Proposition 6.2.5. Suppose $F_{1}, \ldots, F_{s} \in \mathbb{F}_{q}(t)\left[X_{1}, \ldots, X_{r}\right]$ are linear forms defining $V$, and suppose that $\gamma \in \Gamma_{X}\left(\mathbb{F}_{q}(t)\right)$ is defined as in (6.2.4.1). Then the linear polynomials in $\mathbb{F}_{q}(t)\left[X_{0}, \ldots, X_{r}\right]$,

$$
G_{i}:=\left(b_{0}-1\right) F_{i}-F_{i}\left(b_{1}, \ldots, b_{r}\right)\left(X_{0}-1\right), \quad i=1, \ldots, s,
$$

are defining polynomials for $\Gamma_{X}$.

\subsection{Linear relations among Carlitz logarithms.}

6.3.1. Defining polynomials for $Z$. As usual let $Z:=\operatorname{Spec} \Sigma$, where $\Psi=\Psi\left(\alpha_{1}, \ldots, \alpha_{m}\right)$. From Proposition 4.2 .8 we see that $Z$ and $\Gamma_{X}$ are isomorphic over $\overline{k(t)}$. Since $\Gamma_{X}$ is a linear space, $Z$ is also a linear space and isomorphic to $\Gamma_{X}$ over $\bar{k}(t)$. Thus we can pick

$$
\zeta=\left[\begin{array}{cccc}
f_{0} & 0 & \cdots & 0 \\
f_{1} & 1 & \cdots & 0 \\
\vdots & \vdots & \ddots & \vdots \\
f_{r} & 0 & \cdots & 1
\end{array}\right] \in Z(\bar{k}(t))
$$

and then

$$
Z(\bar{k}(t))=\zeta \cdot \Gamma_{X}(\bar{k}(t)) .
$$

It is a simple matter to check that the linear polynomials in $\bar{k}(t)\left[X_{0}, \ldots, X_{r}\right]$,

$$
H_{i}:=G_{i}-X_{0} G\left(f_{0}, \ldots, f_{r}\right) / f_{0}, \quad i=1, \ldots, s,
$$

are defining polynomials for $Z$.

The following theorems show how the above constructions can be used to characterize all $k$-linear relations among $\tilde{\pi}, \log _{C}\left(\alpha_{1}\right), \ldots, \log _{C}\left(\alpha_{r}\right)$.

Theorem 6.3.2. Let $\alpha_{1}, \ldots, \alpha_{r} \in \bar{k}^{\times}$with $\left|\alpha_{i}\right|_{\infty}<|\theta|_{\infty}^{q /(q-1)}$ for $i=1, \ldots, r$. Let $X=X\left(\alpha_{1}, \ldots, \alpha_{r}\right)$ be the associated $t$-motive.

(a) Let $F=c_{1} X_{1}+\cdots+c_{r} X_{r}, c_{1}, \ldots, c_{r} \in \mathbb{F}_{q}(t)$, be a defining linear form for $V$ so that $G=\left(b_{0}-1\right) F-F\left(b_{1}, \ldots, b_{r}\right)\left(X_{0}-1\right), b_{0}, \ldots, b_{r} \in \mathbb{F}_{q}(t), b_{0} \notin \mathbb{F}_{q}$, is a defining polynomial for $\Gamma_{X}$. Then

$$
\left(b_{0}(\theta)-1\right) \sum_{i=1}^{r} c_{i}(\theta) \log _{C}\left(\alpha_{i}\right)-\sum_{i=1}^{r} c_{i}(\theta) b_{i}(\theta) \widetilde{\pi}=0 .
$$

(b) Every $k$-linear relation among $\widetilde{\pi}, \log _{C}\left(\alpha_{1}\right), \ldots, \log _{C}\left(\alpha_{r}\right)$ is a $k$-linear combination of the relations from part (a).

(c) Let $N$ be the $k$-linear span of $\widetilde{\pi}, \log _{C}\left(\alpha_{1}\right), \ldots, \log _{C}\left(\alpha_{r}\right)$. Then $\operatorname{dim} \Gamma_{X}=$ $\operatorname{dim}_{k} N$.

Proof. Choose $f \in \bar{k}(t)$ as in $₫ 6.3 .1$ so that $H:=G-f X_{0}$ is a defining polynomial for $Z$. Then

$$
H\left(\Omega, \Omega L_{\alpha_{1}}, \ldots, \Omega L_{\alpha_{r}}\right)=G\left(\Omega, \Omega L_{\alpha_{1}}, \ldots, \Omega L_{\alpha_{r}}\right)-f \Omega=0 .
$$

We see that

$$
\begin{array}{r}
f^{(-1)} \Omega^{(-1)}=\sigma G\left(\Omega, \Omega L_{\alpha_{1}}, \ldots, \Omega L_{\alpha_{r}}\right)=\Omega G\left(t-\theta-1, \alpha_{1}^{(-1)}(t-\theta), \ldots, \alpha_{r}^{(-1)}(t-\theta)\right) \\
+f \Omega-F\left(b_{1}, \ldots, b_{r}\right) \Omega .
\end{array}
$$


The first equality is a consequence of (6.3.2.1), and the second follows from direct computation. Thus

$$
(t-\theta) f^{(-1)}-f=G\left(t-\theta-1, \alpha_{1}^{(-1)}(t-\theta), \ldots, \alpha_{r}^{(-1)}(t-\theta)\right)-F\left(b_{1}, \ldots, b_{r}\right) .
$$

The right-hand side is a polynomial in $\bar{k}[t]$, so it follows that $f$ is regular at $t=\theta$. Indeed if not, then $f^{(-1)}$ must have a pole at $t=\theta^{(-1)}$, whence $f$ must also have a pole at $t=\theta^{(-1)}$. Continuing in this way we see that if $f$ has a pole at $t=\theta$, then it must have a pole at each $t=\theta^{(-i)}, i \geq 1$, which is not possible. By a similar argument we deduce that $f^{(-1)}$ is also regular at $t=\theta$. Thus we see that

$$
f(\theta)=-\left.G(-1,0, \ldots, 0)\right|_{t=\theta}+\sum_{i=1}^{r} c_{i}(\theta) b_{i}(\theta)=-\sum_{i=1}^{r} c_{i}(\theta) b_{i}(\theta) .
$$

Equation (6.3.2.1) transforms into

$$
\left(b_{0}-1\right) \sum_{i=1}^{r} c_{i} \Omega L_{\alpha_{i}}-\sum_{i=1}^{r} c_{i} b_{i}(\Omega-1)-f \Omega=0 .
$$

Dividing through by $\Omega$ and evaluating at $t=\theta$, we obtain part (a). Part (b) is a consequence of (a) and (c), since $\Gamma_{X}$ is a linear space in $G$ over $\mathbb{F}_{q}(t)$. For part (c), part (a) implies that $\operatorname{dim}_{k} N \leq \operatorname{dim} \Gamma_{X}$, since the defining polynomials for $\Gamma_{X}$ generate a set of $k$-linear relations on $\widetilde{\pi}, \log _{C}\left(\alpha_{1}\right), \ldots, \log _{C}\left(\alpha_{r}\right)$ of dimension $r+1-\operatorname{dim} \Gamma_{X}$. However, $\operatorname{dim}_{k} N \geq \operatorname{tr} . \operatorname{deg}_{\bar{k}} \bar{k}\left(\tilde{\pi}, \log _{C}\left(\alpha_{1}\right), \ldots, \log _{C}\left(\alpha_{r}\right)\right)$ and the latter quantity is $\operatorname{dim} \Gamma_{X}$ by Theorem 5.2 .2

6.3.3. Example. Let $\zeta_{\theta}=\sqrt[q-1]{-\theta}$, let $X$ be the $t$-motive $X\left(\zeta_{\theta}\right)$ of dimension 2 over $\bar{k}(t)$, and let $\Psi=\Psi\left(\zeta_{\theta}\right)$. Since $\zeta_{\theta}$ satisfies $\mathfrak{C}_{t}\left(\zeta_{\theta}\right)=\theta \zeta_{\theta}+\zeta_{\theta}^{q}=0$, we see that $\zeta_{\theta}$ is a $t$-torsion point on the Carlitz module. Moreover, $\exp _{C}\left(\theta \log _{C}\left(\zeta_{\theta}\right)\right)=0$, and one calculates that

$$
\log _{C}\left(\zeta_{\theta}\right)=\frac{\widetilde{\pi}}{\theta}
$$

Thus $\Gamma_{X}$ is 1-dimensional by Theorem 6.3.2(c). If we consider the function in $\mathbb{T}$

$$
\Upsilon:=t L_{\zeta_{\theta}}-\zeta_{\theta}(t-\theta),
$$

then $\Upsilon^{(-1)}=\Upsilon /(t-\theta)$. Thus $\Upsilon=f / \Omega$ for some $f \in \mathbb{F}_{q}[t]$ by Lemma 3.3.5. Evaluation at $t=\theta$ shows that $f=-1$ identically. Therefore, $Z_{\Psi}$ is defined by

$$
Z_{\Psi}: \zeta_{\theta}(t-\theta) X_{0}-t X_{1}-1=0 .
$$

It follows that the defining equation for $\Gamma_{X}$ is

$$
\Gamma_{X}: t X_{1}-X_{0}+1=0 .
$$

In the notation of Theorem 6.3.2, we have

$$
\begin{gathered}
F:=X_{1}, \quad b_{0}:=t+1, \quad b_{1}:=1 \\
G:=t X_{1}-X_{0}+1, \quad H:=G-f X_{0}, \quad f:=\zeta_{\theta}(t-\theta)-1 .
\end{gathered}
$$

6.4. Algebraic independence of Carlitz logarithms. Before proving the main result on Carlitz logarithms, we prove a reduction lemma.

Lemma 6.4.1. Let $\lambda \in \mathbb{K}^{\times}$. If $\exp _{C}(\lambda) \in \bar{k}^{\times}$, then there is an $\alpha \in \bar{k}^{\times}$with $|\alpha|_{\infty}<$ $|\theta|_{\infty}^{q /(q-1)}$, an $f \in \mathbb{F}_{q}[\theta]$, and an $n \geq 1$, so that $\lambda=\theta^{n} \log _{C}(\alpha)+f \widetilde{\pi}$. 
Proof. Let $\beta=\exp _{C}(\lambda)$, and assume that $|\beta|_{\infty} \geq|\theta|_{\infty}^{q /(q-1)}$. We solve the equation $\mathfrak{C}_{t}(x)=\theta x+x^{q}=\beta$; that is, we find the $t$-division points of $\beta$ on the Carlitz module. The Newton polygon for this equation, along with our assumptions on $\beta$, imply that any solution $\alpha \in \bar{k}^{\times}$of this equation must satisfy $|\alpha|_{\infty}=|\beta|_{\infty}^{1 / q}$. Moreover, if for some $\eta \in \mathbb{K}$ we have $\exp _{C}(\eta)=\alpha$, then

$$
\exp _{C}(\theta \eta)=\beta=\exp _{C}(\lambda) .
$$

If $|\beta|_{\infty}<|\theta|_{\infty}^{q^{2} /(q-1)}$, then $\alpha$ is sufficiently small and we can pick $\eta=\log _{C}(\alpha)$. The result then follows with $n=1$. Otherwise, we continue to take $t$-division values, and for some $n \geq 1$, we have $\mathfrak{C}_{t^{n}}(\alpha)=\beta$ with $|\alpha|_{\infty}<|\theta|_{\infty}^{q /(q-1)}$, for which $\exp _{C}\left(\theta^{n} \log _{C}(\alpha)\right)=\beta$.

Theorem 6.4.2. Let $\lambda_{1}, \ldots, \lambda_{r} \in \mathbb{K}$ satisfy $\exp _{C}\left(\lambda_{i}\right) \in \bar{k}$ for $i=1, \ldots, r$. If $\lambda_{1}, \ldots, \lambda_{r}$ are linearly independent over $k$, then they are algebraically independent over $\bar{k}$.

Proof. Assume that $\lambda_{1}, \ldots, \lambda_{r}$ are linearly independent over $k$. By Lemma 6.4.1, for each $\lambda_{i}$ we can pick $\alpha_{i} \in \bar{k}^{\times}$with $\left|\alpha_{i}\right|_{\infty}<|\theta|_{\infty}^{q /(q-1)}$ so that the $k$-linear span of $\lambda_{1}, \ldots, \lambda_{r}$ is contained in the $k$-linear span of $\widetilde{\pi}, \log _{C}\left(\alpha_{1}\right), \ldots, \log _{C}\left(\alpha_{r}\right)$. Let $X=$ $X\left(\alpha_{1}, \ldots, \alpha_{r}\right)$ be the $t$-motive associated to these logarithms as in the previous sections, and let $\Gamma_{X}$ be its Galois group. Let

$$
L=\bar{k}\left(\widetilde{\pi}, \log _{C}\left(\alpha_{1}\right), \ldots, \log _{C}\left(\alpha_{r}\right)\right)
$$

and let

$$
N=k \text {-linear span of } \widetilde{\pi}, \log _{C}\left(\alpha_{1}\right), \ldots, \log _{C}\left(\alpha_{r}\right) \text {. }
$$

Because $\lambda_{1}, \ldots, \lambda_{r}$ are linearly independent over $k$, we see that $r \leq \operatorname{dim}_{k} N \leq r+1$. Theorems 5.2 .2 and 6.3 .2 imply that

$$
\text { tr. } \operatorname{deg}_{\bar{k}} L=\operatorname{dim} \Gamma_{X}=\operatorname{dim}_{k} N .
$$

If $\widetilde{\pi}, \log _{C}\left(\alpha_{1}\right), \ldots, \log _{C}\left(\alpha_{r}\right)$ are linearly independent over $k$, then they are algebraically independent over $\bar{k}$, whence the same follows for $\lambda_{1}, \ldots, \lambda_{r}$ since $L=\bar{k}\left(\widetilde{\pi}, \lambda_{1}, \ldots, \lambda_{r}\right)$. If there is a linear dependence among $\widetilde{\pi}, \log _{C}\left(\alpha_{1}\right), \ldots, \log _{C}\left(\alpha_{r}\right)$ over $k$, then $N$ is equal to the $k$-span of $\lambda_{1}, \ldots, \lambda_{r}$ and $L=\bar{k}\left(\lambda_{1}, \ldots, \lambda_{r}\right)$. Thus in that case $\lambda_{1}, \ldots, \lambda_{r}$ are algebraically independent over $\bar{k}$.

\section{REFERENCES}

[1] G. W. Anderson, t-motives, Duke Math. J. 53 (1986), 457-502.

[2] G. W. Anderson, W. D. Brownawell, and M. A. Papanikolas, Determination of the algebraic relations among special $\Gamma$-values in positive characteristic, Ann. of Math. (2) 160 (2004), 237313 .

[3] G. W. Anderson and D. S. Thakur, Tensor powers of the Carlitz module and zeta values, Ann. of Math. (2) 132 (1990), 159-191.

[4] Y. André, Différentielles non commutatives et théorie de Galois différentielle ou aux différences, Ann. Sci. École Norm. Sup. (4) 34 (2001), 685-739.

[5] C. Bertolin, Périodes de 1-motifs et transcendance, J. Number Theory 97 (2002), 204-221.

[6] F. Beukers, Differential Galois theory, From number theory to physics (Les Houches, 1989), Springer, Berlin, 1992, pp. 413-439.

[7] G. Böckle and U. Hartl, Uniformizable families of $t$-motives, 2004, preprint, math.NT/0411262

[8] L. Breen, Tannakian categories, Motives (Seattle, WA, 1991), Proc. Sympos. Pure Math., vol. 55, part 1, Amer. Math. Soc., Providence, RI, 1994, pp. 337-376.

[9] W. D. Brownawell, Transcendence in positive characteristic, Number theory (Tiruchirapalli, 1996), Contemp. Math., vol. 210, Amer. Math. Soc., Providence, RI, 1998, pp. 317-332.

[10] P. Deligne, Catégories tannakiennes, The Grothendieck Festschrift, Vol. II, Progr. Math., vol. 87, Birkhäuser, Boston, MA, 1990, pp. 111-195.

[11] P. Deligne, J. S. Milne, A. Ogus, and K.-Y. Shih, Hodge cycles, motives, and Shimura varieties, Lecture Notes in Mathematics, vol. 900, Springer-Verlag, Berlin, 1982. 
[12] L. Denis, Independence algébrique de logarithmes en caractéristique p, Bull. Austral. Math. Soc. $\mathbf{7 4}$ (2006), 461-470.

[13] J. Fresnel and M. van der Put, Rigid analytic geometry and its applications, Birkhäuser, Boston, 2004.

[14] D. Goss, Drinfeld modules: cohomology and special functions, Motives (Seattle, WA, 1991), Proc. Sympos. Pure Math., vol. 55, part 2, Amer. Math. Soc., Providence, RI, 1994, pp. 309-362.

[15] D. Goss, Basic structures of function field arithmetic, Springer-Verlag, Berlin, 1996.

[16] U. Hartl and R. Pink, Vector bundles with a Frobenius structure on the punctured unit disc, Compos. Math. 140 (2004), 689-716.

[17] K. S. Kedlaya, The algebraic closure of the power series field in positive characteristic, Proc. Amer. Math. Soc. 129 (2001), 3461-3470.

[18] E. R. Kolchin, Differential algebra and algebraic groups, Academic Press, New York, 1973.

[19] S. Lang, Algebraic groups over finite fields, Amer. J. Math. 78 (1956), 555-563.

[20] A. R. Magid, Lectures on differential Galois theory, University Lecture Series, vol. 7, Amer. Math. Soc., Providence, RI, 1994.

[21] H. Matsumura, Commutative algebra, 2nd ed., Benjamin/Cummings Publ., Reading, Mass., 1980.

[22] H. Matsumura, Commutative ring theory, Cambridge University Press, Cambridge, 1986.

[23] R. Pink, Hodge structures for function fields, 1997, preprint, http://www.math.ethz.ch/ pink/

[24] M. van der Put, Galois theory of differential equations, algebraic groups and Lie algebras, J. Symbolic Comput. 28 (1999), 441-472.

[25] M. van der Put and M. F. Singer, Galois theory of difference equations, Lecture Notes in Mathematics, vol. 1666, Springer-Verlag, Berlin, 1997.

[26] M. van der Put and M. F. Singer, Galois theory of linear differential equations, Springer-Verlag, Berlin, 2003.

[27] P. Ribenboim, Fields: algebraically closed and others, Manuscripta Math. 75 (1992), 115-150.

[28] S. K. Sinha, Periods of t-motives and transcendence, Duke Math. J. 88 (1997), 465-535.

[29] Y. Taguchi, The Tate conjecture for t-motives, Proc. Amer. Math. Soc. 123 (1995), 3285-3287.

[30] A. Tamagawa, The Tate conjecture and the semisimplicity conjecture for $t$-modules, Sūrikaisekikenkyūsho Kōkyūroku (1995), no. 925, 89-94.

[31] D. S. Thakur, Function field arithmetic, World Scientific Publishing, River Edge, NJ, 2004.

[32] W. C. Waterhouse, Introduction to affine group schemes, Springer-Verlag, New York, 1979.

[33] J. Yu, Analytic homomorphisms into Drinfeld modules, Ann. of Math. (2) 145 (1997), 215-233.

[34] O. Zariski and P. Samuel, Commutative algebra. Vol. II, Springer-Verlag, New York, 1975.

Department of Mathematics, Texas A\&M University, College Station, TX 77843

E-mail address: map@math.tamu.edu 